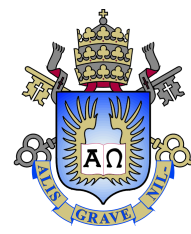

Nicolle Miranda de Lima

\title{
Microscale Analysis of Foam Formation and Surfactant-Alternating-Gas Injection in Porous \\ Media Micromodels
}

Tese de Doutorado

Thesis presented to the Programa de Pós-graduação em Engenharia Mecânica of PUC-Rio in partial fulfillment of the requirements for the degree of Doutor em Engenharia Mecânica.

Advisor: Prof. Márcio da Silveira Carvalho 

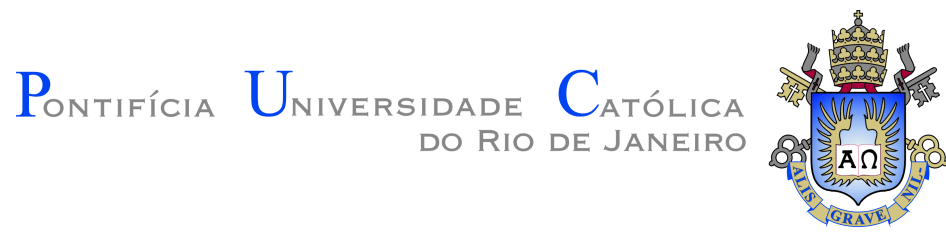

Nicolle Miranda de Lima

\section{Microscale Analysis of Foam Formation and Surfactant-Alternating-Gas Injection in Porous Media Micromodels}

Thesis presented to the Programa de Pós-graduação em Engenharia Mecânica of PUC-Rio in partial fulfillment of the requirements for the degree of Doutor em Engenharia Mecânica. Approved by the Examination Committee.

Prof. Márcio da Silveira Carvalho Advisor Departamento de Engenharia Mecânica - PUC-Rio

Prof. Aurora Pérez Gramatges Departamento de Química - PUC-Rio

Prof. Grigori Chapiro

Departamento de Matemática - UFJF

Prof. Shima Parsa

School of Physics and Astronomy - Rochester Institute of Technology

Prof. Vladimir Alvarado Basante

Department of Chemical Engineering - University of Wyoming 
All rights reserved.

\section{Nicolle Miranda de Lima}

Nicolle Lima received a bachelor's degree in Petroleum Engineering from Universidade Federal Fluminense(UFF) in 2013 and a master's degree in Mechanical Engineering from PUCRio in 2015. Currently, she works as a researcher at the Laboratory of Microhydrodynamics and Flow in Porous Media at PUC-Rio, while pursuing a $\mathrm{PhD}$ degree at the same institution, in the Mechanical Engineering Department. In 2018, she spent one year at Harvard University in the Experimental Soft Condensed Matter group cordinated by David Weitz under a Fulbright Scholarship with the aim of developing part of her $\mathrm{PhD}$ research.

Bibliographic data

Lima, Nicolle Miranda de

Microscale Analysis of Foam Formation and SurfactantAlternating-Gas Injection in Porous Media Micromodels / Nicolle Miranda de Lima; advisor: Márcio da Silveira Carvalho. $-2021$.

101 f.: il. color. ; $30 \mathrm{~cm}$

Tese (doutorado) - Pontifícia Universidade Católica do Rio de Janeiro, Departamento de Engenharia Mecânica, 2021

Inclui bibliografia

1. Engenharia Mecânica - Teses. 2. Espuma;. 3. Meios Porosos;. 4. Micromodelo;. 5. Microfluídica;. 6. Recuperação Avançada de Óleo;. I. Carvalho, Márcio. II. Pontifícia Universidade Católica do Rio de Janeiro. Departamento de Engenharia Mecânica. III. Título. 


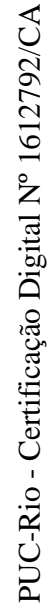

I dedicate this work to my family. 


\section{Acknowledgments}

To my friends and family, for their support and inspiration.

To my advisor, Prof. Márcio Carvalho, for his trust and excellent technical guidance. His vast knowledge and experience has encouraged me throughout all my academic research.

To Professor Shima Parsa, for assisting me during my stay at Harvard University and for being a source of inspiration.

To Professor David Weitz, for receiving me at Harvard University and whose sharp feedback pushed me to strengthen my science (and food) understanding.

To Professor Sidnei Paciornik, for his patience and help with the image processing. His knowledge and dedication helped me through the most challenging part of this thesis.

To my laboratory colleagues at LMMP for the valuable discussions and all the help through these years. Also for the friendship and good times shared during these years.

To Fulbright, Harvard University, ANP, CNPq, CAPES and PUC-Rio, for the funding without which this research could not have been accomplished.

This study was financed in part by the Coordenação de Aperfeiçoamento de Pessoal de Nível Superior - Brasil (CAPES) - Finance Code 001. 


\section{Abstract}

Lima, Nicolle; Carvalho, Márcio (Advisor). Microscale Analysis of Foam Formation and Surfactant-Alternating-Gas Injection in Porous Media Micromodels. Rio de Janeiro, 2021. 101p. Tese de doutorado - Departamento de Engenharia Mecânica, Pontifícia Universidade Católica do Rio de Janeiro.

Foam is widely used in oil recovery operations to improve sweep efficiency, in gas storage and acidization operations, and to solve problems caused by either a thief zone or gravity override. Foam, which can be preformed and injected into the reservoir or produced in situ through the pore space, fills the high permeability areas known as thief zones and diverts the displacing fluid into the direction of trapped oil, reducing the relative permeability of gas and leading to a more stable displacement front. The efficiency of these processes largely depends on the generation and stability of the foam films (lamellae) residing in the pores. The mobility of the injected gas is reduced when foam is formed; this reduction is attributed to the reduction of the gas phase relative permeability. The lamellae formed create resistance against the gas flow, impeding its free motion inside the porous media. The lamellae population that composes the foam is directly related to surfactant concentration, and their flow and mobility are functions of the pore geometry and foam properties. However, the dynamics of foam formation in porous media is not fully understood due to its complexity. The goal of the first part of this research is to understand the impact of increasing surfactant concentration on foam formation during gas injection in a two-dimensional porous media glass model occupied by a surfactant solution. The second part focuses on foam formation and its implications for oil displacement during the SAG (surfactant-alternating-gas) injection, considering different surfactant concentrations. A microfluidic setup composed of a glass micromodel, syringe pump, pressure transducer and microscope, was used to visualize the pore-scale displacement and correlate the evolution of lamellae formation during the injection process with pressure difference for different flow conditions through image processing. The dynamics of lamellae formation is reported and related to macroscopic flow behavior.

\section{Keywords}

Foam; Porous Media; Micromodel; Microfluidics; Enhanced Oil Recovery; 


\section{Resumo}

Lima, Nicolle; Carvalho, Márcio. Análise em Microescala da Formação de Espuma e Injeção Alternada de Surfactante e Gás em Micromodelos de Meios Porosos. Rio de Janeiro, 2021. 101p. Tese de Doutorado - Departamento de Engenharia Mecânica, Pontifícia Universidade Católica do Rio de Janeiro.

A espuma é amplamente usada em operações de recuperação de óleo para melhorar a eficiência de varrido, em operações de armazenamento de gás e acidificação, e para resolver problemas causados por zonas ladras ou segregação gravitacional. A espuma, que pode ser pré-formada e injetada no reservatório ou produzida in situ através da geometria do meio poroso, escoa nas regiões de alta permeabilidade e desvia o fluido de deslocamento na direção do óleo aprisionado, reduzindo a permeabilidade relativa ao gás e levando a uma frente de deslocamento mais estável. A eficiência desses processos depende muito da geração e estabilidade dos filmes de espuma (lamelas) que residem nos poros. A mobilidade do gás injetado é reduzida quando a espuma é formada; esta redução é atribuída ao aumento da viscosidade efetiva do gás e à redução da permeabilidade relativa ao gás. As lamelas formadas criam resistência ao fluxo do gás, impedindo seu movimento livre dentro do meio poroso. A população de lamelas que compõe a espuma está diretamente relacionada com a concentração de surfactante, e seu fluxo e mobilidade são funções da geometria dos poros e das propriedades da espuma. No entanto, a dinâmica da formação de espuma em meios porosos não é totalmente compreendida devido à sua complexidade O objetivo da primeira parte desta pesquisa é compreender o impacto do aumento da concentração de surfactante na formação de espuma durante a injeção de gás em um modelo bidimensional de meio poroso de vidro saturado com uma solução de surfactante. A segunda parte foca na formação de espuma e sua implicação no deslocamento de óleo durante o processo de injeção SAG (injeção alternada de solução de surfactante e gás) considerando diferentes concentrações de surfactante. Uma configuração microfluídica composta por micromodelo de vidro, bomba de seringa, transdutor de pressão e microscópio foi usada para visualizar o deslocamento da escala dos poros e correlacionar a evolução da formação das lamelas durante o processo de injeção com a diferença de pressão para diferentes condições de fluxo através do processamento de imagem. A dinâmica de formação das lamelas é relatada e relacionada ao comportamento do fluxo macroscópico.

\section{Palavras-chave}

Espuma; Meios Porosos; Micromodelo; Microfluídica; Recuperação Avançada de Óleo; 


\section{Table of contents}

$\begin{array}{llr}1 & \text { Introduction } & \mathbf{1 5}\end{array}$

$\begin{array}{lll}1.1 & \text { Oil production } & 15\end{array}$

$\begin{array}{lll}1.2 & \text { Enhanced oil recovery } & 17\end{array}$

$\begin{array}{lll}1.3 & \text { Foam Sweep Efficiency } & 19\end{array}$

1.4 Foam Generation in Oil Industry 20

$\begin{array}{ll}1.5 & \text { Surfactant-alternating-gas injection } \\ 1.6 & 22\end{array}$

1.6 Outline 23

2 Literature review and thesis goals $\quad 24$

2.1 Foam 24

2.2 Foam generation 25

2.2.0.1 Leave behind 25

2.2.0.2 Lamella division 26

2.2.0.3 Snap-off 26

2.2.0.4 Pinch-off 28

2.2.1 Regimes of foam flow 28

2.2.2 Critical pressure gradient and rate of foam generation 29

2.2.3 Lamella-destruction mechanism 31

2.2.3.1 Coarsening 31

2.2.3.2 Capillary suction $\quad 32$

2.3 Foam drainage and stability 35

$\begin{array}{lll}2.4 & \text { Flow in porous media } & 37\end{array}$

2.5 Foam as an EOR method 40

$\begin{array}{lll}2.6 & \text { Objectives } & 46\end{array}$

3 Materials and methods $\quad 47$

$\begin{array}{ll}3.1 \text { Materials } & 47\end{array}$

3.1.1 Surfactant 47

3.1.2 Air 51

3.1.3 Oil 51

3.2 Porous Media Micromodel $\quad 53$

4 Foam formation during drainage of a surfactant solution $\quad 55$

4.1 Experimental setup and procedures $\quad 55$

4.2 Image Processing $\quad 57$

$\begin{array}{lll}4.3 & \text { Results } & 61\end{array}$

4.3.1 Gas displacing pure water 61

4.3.2 Foam formation in the surfactant solution displacement experiments 62

$\begin{array}{lll}\text { 4.3.3 Reproducibility } & 75\end{array}$

4.3.4 Effect of injection flow rate $\quad 76$

5 Oil displacement by surfactant-alternating-gas (SAG) injection $\quad 79$

5.1 Experimental setup and procedure $\quad 79$

5.2 Image processing 80 
$\begin{array}{lll}5.3 & \text { Results } & 84\end{array}$

$\begin{array}{ll}\text { 5.3.0.1 Reproducibility } & 91\end{array}$

6 Conclusions $\quad 93$

6.1 Foam formation during drainage of a surfactant solution 93

6.2 Oil displacement by surfactant-alternating-gas (SAG) injection 94

$\begin{array}{lll}6.3 & \text { Future work } & 95\end{array}$

$\begin{array}{lr}\text { Bibliography } & 96\end{array}$ 


\section{List of figures}

$\begin{array}{lll}\text { Figure 1.1 Waterflooding scheme } & 16\end{array}$

$\begin{array}{lll}\text { Figure 1.2 Viscous fingering formed during waterflooding } & 17\end{array}$

$\begin{array}{lll}\text { Figure 1.3 Sweep efficiency for different injection methods } & 18\end{array}$

$\begin{array}{lll}\text { Figure 1.4 Mechanisms affecting apparent viscosity } & 20\end{array}$

Figure 1.5 Foam placement strategies in EOR projects 21

Figure 1.6 Displacing front for gas, WAG and SAG 22

Figure 2.1 Foam aspects for different liquid fractions 24

$\begin{array}{lll}\text { Figure 2.2 Dry foam } & 25\end{array}$

Figure 2.3 Leave behind 26

Figure 2.4 Lamella division 26

Figure 2.5 Snap-off mechanism. Black is the pore-throat wall, gray $\begin{array}{ll}\text { is the water and white is the gas } & 27\end{array}$

Figure 2.6 Roof Snap-off as gas invades a pore body. Same color scheme as previous picture 27

Figure 2.7 Pinch-off mechanism: the pink bubble pinches the green bubble against the post, forming a new bubble 28

Figure 2.8 Concepts of weak and strong foams within porous media 29

Figure 2.9 Pressure gradient as a function of interstitial velocity 30

Figure 2.10 Coarsening 31

Figure 2.11 Foam coarsening mechanism can be observed in a glass micromodel. In (a) at the beginning of the experiment $t=0$ and in (b) after 2000 seconds

Figure 2.12 Minimum-energy configuration of the lamella in the pore throat

Figure 2.13 Scheme of capillary suction inducing film thinning 33

Figure 2.14 Disjoining pressure isotherm 34

Figure 2.15 Hydrodynamic lifetime of foam films versus surfactant $\begin{array}{ll}\text { concentration } & 36\end{array}$

Figure 2.16 Different forms that gas can take in porous media 38

Figure 2.17 Flow-focusing device and micromodel showing permeability contrast 40

Figure 2.18 Foam sweep efficiency comparing with water and WAG 40

Figure 2.19 Recovery factors of SAG, WAG, water injection and gas injection

Figure 2.20 Pressure gradient across the sample at two different concentrations

Figure 2.21 Porous media device used by Wang (oil was dyed in yellow, water in blue and gas remains transparent)

Figure 2.22 Bubble recognition a) Device saturated with surfactant solution without bubbles b) Micromodel with coarse foam c) Micromodel with fine foam

Figure 2.23 (a) Lamellae number as a function of time for different gas flow rates (b) Pressure $\mathrm{x}$ injected pore volume (PV)

Figure 2.24 Setup of the model fracture showing the six sections 
Figure 2.25 Evolution of bubble density and sizes at different sections along the fracture

$\begin{array}{lll}\text { Figure 3.1 Sodium dodecyl sulfate (SDS) } & 48\end{array}$

Figure 3.2 DataPhysics Tensiometer 48

Figure 3.3 Surface tension as a function of concentration 49

Figure 3.4 Air-SDS solution interfacial tension 49

Figure 3.5 Air-SDS interfacial tension near CMC 50

Figure 3.6 Oil and surfactant solution used for interfacial tension measurement

Figure 3.7 Oil-surfactant interfacial tension $\quad 52$

$\begin{array}{lll}\text { Figure } 3.8 & \text { Micronit microfluidic device } & 54\end{array}$

Figure 4.1 Scheme of the experimental setup $\quad 55$

$\begin{array}{lll}\text { Figure 4.2 Experimental setup } & 56\end{array}$

$\begin{array}{lll}\text { Figure 4.3 The area analyzed in the micromodel } & 57\end{array}$

$\begin{array}{lll}\text { Figure 4.4 Device filled with air } & 58\end{array}$

Figure 4.5 Device filled with liquid films (lamellae) 58

$\begin{array}{lll}\text { Figure 4.6 Image processing flowchart } & 59\end{array}$

$\begin{array}{lll}\text { Figure 4.7 Binary images } & 60\end{array}$

$\begin{array}{lll}\text { Figure } 4.8 & \text { A)Lamellae identified by subtraction B)Nodes } & 60\end{array}$

Figure 4.9 Lamellae marked in red 61

Figure 4.10 Normalized pressure behavior during gas injection - no surfactant added $\quad 62$

Figure 4.11 Phase distribution after gas injection - no surfactant added 62

Figure 4.12 Leave behind - A) adjacent pore bodies saturated with surfactant solution - B) gas invasion - C) leave-behind lamellae 63

Figure 4.13 Lamella division

Figure 4.14 Snap-off - A original bubble (red) - B gas-filled pore throat - C new bubble (blue) created after snap-off

Figure 4.15 Pinch-off - A - bubbles enter pore throat - B red bubble squeezed by yellow bubble - C bubble pinched add lamellae (blue) 64

Figure 4.16 A three-sided cell marked with a red circle disappeared due to diffusion

Figure 4.17 Normalized pressure behavior during gas injection

Figure 4.18 Phase distribution after gas injection - surfactant concentration below CMC (c = $0.235 \mathrm{~g} / \mathrm{L})$

Figure 4.19 Normalized pressure behavior during gas injection surfactant concentrations below the CMC

Figure 4.20 Normalized pressure behavior during gas injection $\quad 67$

Figure 4.21 Maximum pressure for different concentrations 68

Figure 4.22 Time when the maximum pressure was reached for different concentrations

Figure 4.23 Pressure at steady state condition for different concentrations

Figure 4.24 Liquid fraction at the beginning of the experiment (A) and at the end $(\mathrm{B}) \mathrm{c}=15.5 \mathrm{~g} / \mathrm{L}$

Figure 4.25 Phase distribution after gas injection - surfactant concentration below the $\mathrm{CMC} \mathrm{c}=0.47 \mathrm{~g} / \mathrm{L}$ 
Figure 4.26 Phase distribution after gas injection - surfactant concentration near the $\mathrm{CMC} \mathrm{c}=1.18 \mathrm{~g} / \mathrm{L}$

Figure 4.27 Phase distribution after gas injection - surfactant concentration well above the $\mathrm{CMC} \mathrm{c}=15.5 \mathrm{~g} / \mathrm{L}$

Figure 4.28 Normalized pressure and lamellae number per millimeter square as a function of time (concentration $=3.1 \mathrm{~g} / \mathrm{L}$ and gas flow rate $=1 \mathrm{ml} / \mathrm{h}$ )

Figure 4.29 Normalized pressure and lamellae number per millimeter square as a function of time (concentration $=15.5 \mathrm{~g} / \mathrm{L}$ and gas flow rate $=1 \mathrm{ml} / \mathrm{h}$ )

Figure 4.30 Lamellae number per millimeter square vs time

Figure 4.31 Maximum lamellae density vs surfactant solution concentration

Figure 4.32 Micromodel divided in four quarters (Q1, Q2, Q3 and Q4) 74

Figure 4.33 Total lamellae number for each concentration as a function of the four regions studied

Figure 4.34 Normalized pressure for low surfactant concentration (green curves) and high surfactant concentration (dark red curves) as a function of time

Figure 4.35 Lamellae number per millimeter square for low surfactant concentration (green curves) and high surfactant concentration (dark red curves) as a function of time

Figure 4.36 Pressure as a function of time during gas injection different gas flow rates

Figure 4.37 Normalized pressure as a function of time during gas injection - different gas flow rates

Figure 4.38 Normalized pressure at steady state conditions for different flow rates

Figure 5.1 Scheme of the experimental setup composed by a syringe pump, a pressure transducer, the micromodel, a stereoscopic microscope and a computer to do the image processing

Figure 5.2 Surfactant solution injection in the micromodel saturated with oil

Figure 5.3 Micromodel saturated with oil (A) Original image (B) Mask

Figure 5.4 Image threshold for the mask

Figure 5.5 Fluid distribution after aqueous solution injection. Oil appears in brown and surfactant solution in red

Figure 5.6 Binary image after aqueous solution injection. The darkest regions correspond to the oil phase

Figure 5.7 Image threshold

Figure 5.8 Oil phase identified and marked in red after surfactant solution injection

Figure 5.9 Oil phase identified and marked in red after surfactant solution and gas injection

Figure 5.10 Fluid saturation after (A) Pure water injection (B) Gas injection

Figure 5.11 Normalized pressure during gas injection 
Figure 5.12 Phase distribution after (A) Surfactant solution injection $(\mathrm{c}=0.47 \mathrm{~g} / \mathrm{L})(\mathrm{B})$ Gas injection

Figure 5.13 Normalized pressure during gas injection (SDS concentration $\mathrm{c}=0.47 \mathrm{~g} / \mathrm{L})$

Figure 5.14 Phase distribution after (A) Surfactant solution injection $(\mathrm{c}=6.2 \mathrm{~g} / \mathrm{L})(\mathrm{B})$ Gas injection

Figure 5.15 Normalized pressure during gas injection (SDS concentration $\mathrm{c}=6.2 \mathrm{~g} / \mathrm{L}$ )

Figure 5.16 Phase distribution after (A) Surfactant solution injection $(\mathrm{c}=15.5 \mathrm{~g} / \mathrm{L})(\mathrm{B})$ Gas injection

Figure 5.17 Normalized pressure during gas injection (SDS concentration $\mathrm{c}=15.5 \mathrm{~g} / \mathrm{L})$

Figure 5.18 Normalized pressure during gas injection for different concentrations after surfactant solution injection

Figure 5.19 Normalized pressure for two different tests at the same concentration as a function of time 


\section{List of tables}

Table 3.1 Dynamic viscosity for different surfactant concentrations 51

Table 4.1 Experimental values to ratify the test reproducibility $\quad 76$

Table $5.1 \%$ of oil available after each step for different concentrations 91

Table $5.2 \%$ of oil available after each step for different experiments

at the same concentration $\quad 92$ 


\section{Introduction}

\section{1}

\section{Oil production}

Petroleum is a fossil fuel created by the decomposition of ancient organisms. Over millions of years and under specific heat and pressure conditions, organic matter was transformed into carbon-rich fluids. These hydrocarbons were physically trapped in oil reservoirs, porous rock formations, typically sandstones or carbonates. The oil industry's greatest challenge is to more efficiently extract oil from these reservoirs.

There are three main types of oil recovery processes. Primary recovery refers to the initial stage in the oil extraction process. In this method, natural pressure from the reservoir or gravity drives oil towards the wellbore. When the natural reservoir energy is depleted, an external source of energy must be supplied. The injection of fluids, such as natural gas or water, is commonly used to accomplish this. It is a process called secondary oil recovery. Waterflooding is the term used when water injection is used as a secondary recovery method, and is illustrated in Figure 1.1. The fundamental goal of either natural gas or water injection operation is to maintain the reservoir at a high pressure and displace oil towards the producing wells. There are many advantages in waterflooding method, for example, the extensive knowledge of the technique, fluid availability, and the low cost compared with other methods. 


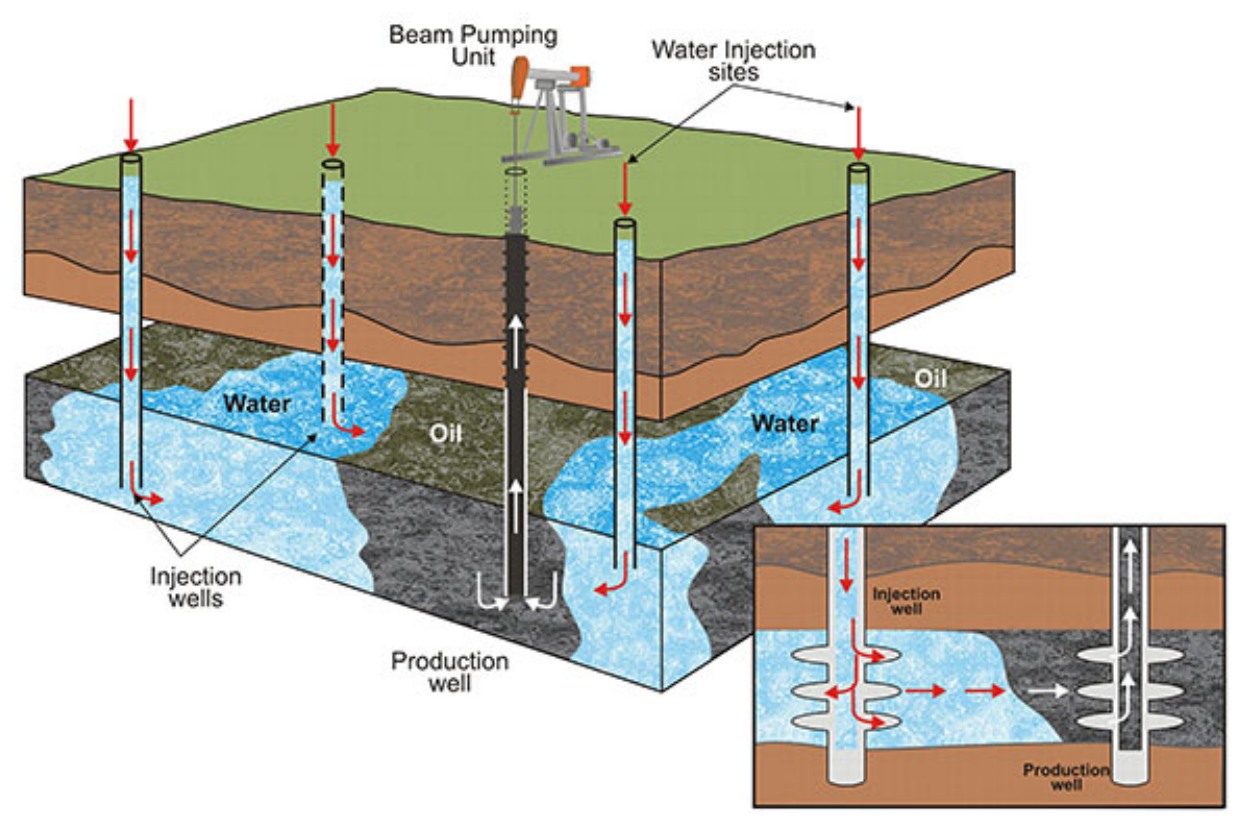

Figure 1.1: Waterflooding scheme [1]

During water injection process, water moves as a bank of fluid through the reservoir, pushing oil ahead of it. The recovery efficiency of waterflooding is determined by both the macroscopic sweep efficiency and the residual oil saturation in the areas swept by the injected phase. Sweep efficiency refers to how well the water contacts the available pore space in the oil-bearing zone. Low sweep efficiency is caused by large heterogeneities in the rock matrix and flow instabilities. Heterogeneities include fractures, high-permeability streaks, and faults. Sweep efficiency is higher in homogeneous rock formations.

Another key factor in sweep efficiency is the water-to-oil mobility ratio, defined as:

$$
M=\frac{k_{r_{D}} / \mu_{D}}{k_{r_{d}} / \mu_{d}}
$$

where $k_{r D}$ and $\mu_{D}$ are the relative permeability and viscosity of the displacing phase, respectively, and $k_{r_{d}}$ and $\mu_{d}$ represent the displaced phase. Mobility ratios less than 1 indicate favorable displacement. High mobility ratios result in low sweep efficiency, as sketched in Figure 1.2, as well as early injected water breakthrough. 


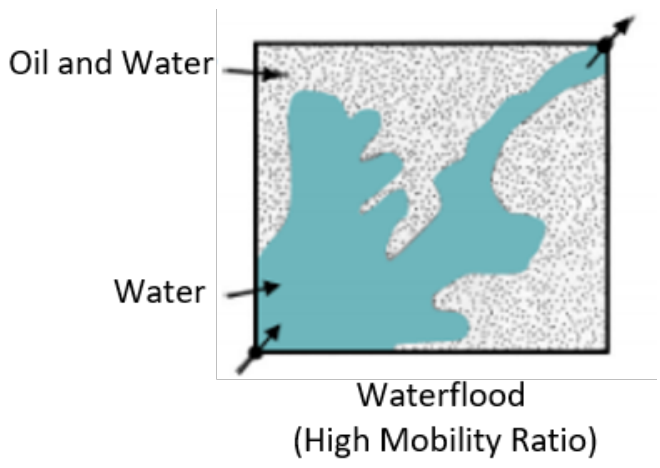

Figure 1.2: Viscous fingering formed during waterflooding. Adapted from [2]

As mentioned before, the amount of oil displaced is also a function of how well oil is displaced at the pore scale. The volume of trapped oil ganglia in the pore space varies with the relative magnitude of the viscous to surface tension forces acting across an interface between a liquid and a gas, or between two immiscible liquids. This ratio is represented by the capillary number that can be defined as

$$
C a=\frac{\mu V}{\sigma}
$$

where $\mu$ is the dynamic viscosity of the liquid, $V$ is a characteristic velocity and $\sigma$ is the interfacial tension between the two phases.

Capillary forces play an important role in trapping oil in porous media and oil can be efficiently displaced if viscous forces dominate against capillary forces. The residual oil saturation falls as capillary number rises.

\section{2}

Enhanced oil recovery

When the secondary recovery becomes ineffective, Enhanced Oil Recovery (EOR) methods are applied. The idea is to maximize oil production through the interaction between injected fluid and the rock/oil system. Increasing the recovery factor, which is a function of the displacement mechanisms, is a key goal of Enhanced Oil Recovery. The term tertiary recovery is no longer used due to the fact that EOR methods can be used at the beginning or at any time during the oil recovery process.

Miscible, chemical, thermal, and microbial flooding techniques are the four types of enhanced oil recovery processes. In miscible flooding, miscible gases are injected into the reservoir and act as solvents. A mixing zone forms between the displacing fluid and the reservoir oil, creating a piston-like displacement. In a miscible flooding process, the interfacial tension is reduced 
to zero. Microorganisms are used in microbial processes to improve oil recovery. They react with a carbon source to produce surfactants, polymers, biomass, gases, solvents, and organic acids. Oil recovery mechanisms are similar in chemical methods, the main goals are oil viscosity reduction, interfacial tension reduction, wettability alteration, selective plugging, and increase in reservoir permeability. Thermal methods are excellent for heavy crude oil reservoirs and involve hot water, steam or in situ combustion. It is the most advanced EOR method category in terms of technology and field experience. Thermal methods provide heat to the reservoir and vaporize some of the oil. The chemical processes cause a decrease in mobility ratio and/or an increase in capillary number, and include alkaline flooding, polymer, micellar-polymer, and foam $[3,4]$.

Gas drive fluids for enhanced oil recovery, like carbon dioxide, nitrogen and steam, have very low viscosity and consequently high mobility, causing them to flow through preferential flow paths rather than efficiently sweeping the reservoir. This gas channeling causes an early breakthrough. The formation of preferential flow paths is amplified by gravity segregation, which occurs because of the low density of the gas compared to other fluids present in the reservoir. The displacement front of gas injection is not stable and, in most cases, gas flows through the upper part of the reservoir, leaving a large amount of oil behind, sketched in Figure 1.3.

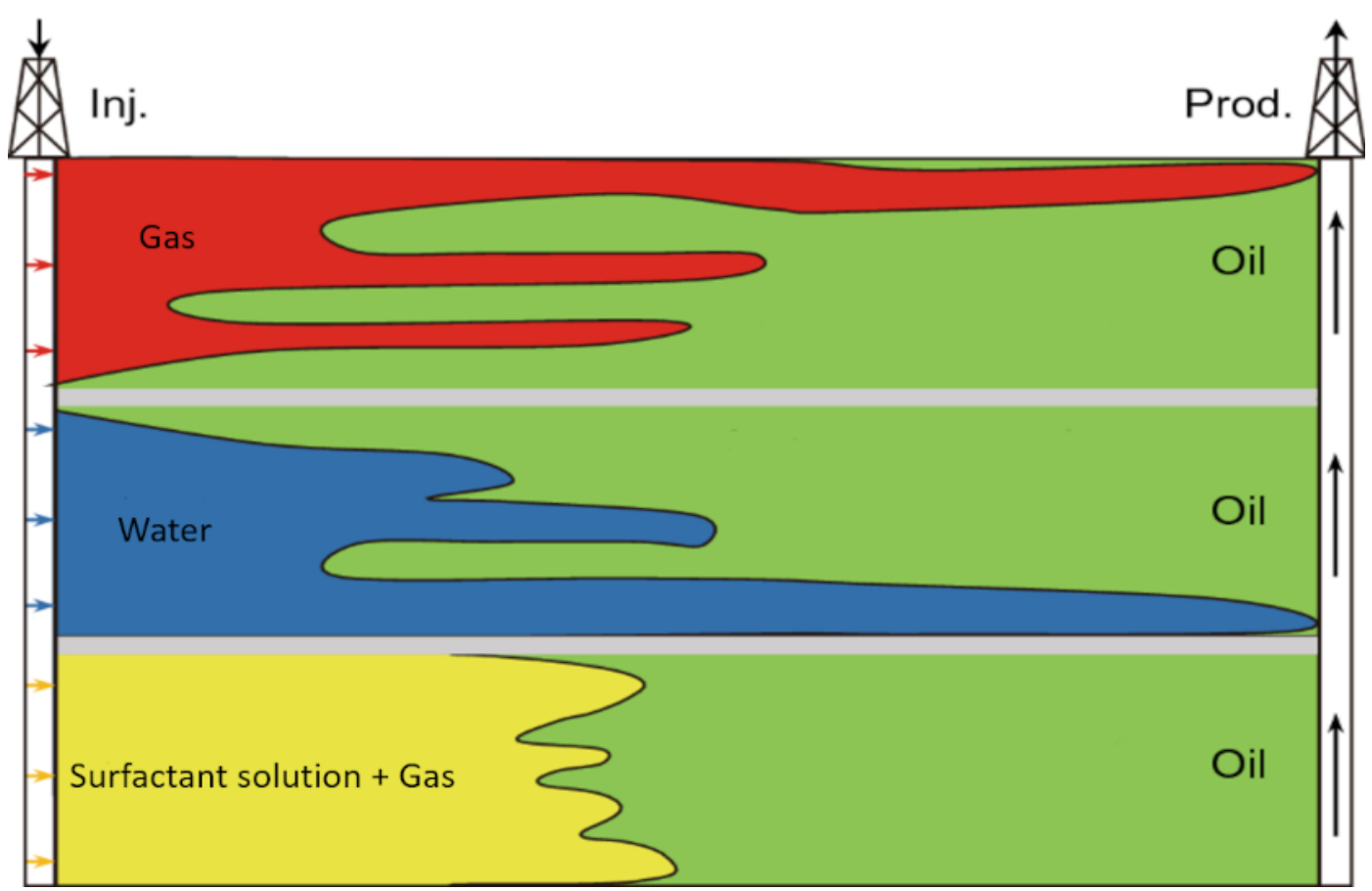

Figure 1.3: Sweep efficiency for different injection methods. Adapted from [5]

Poor sweep efficiency and macroscopic displacement instabilities can 
be overcome with foam injection, as sketched in the bottom of Figure 1.3. Foam can be injected into the subsurface or formed in situ during surfactantalternating-gas injection to reduce the mobility of the gas phase by increasing its effective viscosity and to divert the gas flow to low permeability regions ([6]; [7]). It is a technique that has been successfully used as an EOR method and in the remediation of contaminated sites ([8]; [9]; [10]). Its success highly depends on generation and stability of foam residing in the pores.

Because of its higher apparent viscosity compared to gases like carbon dioxide, nitrogen, air and hydrocarbon gases, foam can reduce the risks of channeling, viscous fingering and gravity override that usually happen in heterogeneous reservoirs [11]. The significant reduction of gas mobility can effectively maintain a stable displacement front and therefore increase the efficiency of gas sweep and oil recovery compared to exclusively gas and water two-phase flow.

Foam decreases the mobility ratio $M$ by decreasing the gas relative permeability achieved by trapping gas in bubbles and increasing the effective viscosity due to the lamellae resistance to flow. In the case of surfactant-alternategas injection, the surfactant solution can significantly reduce interfacial tension between the aqueous and oil phases.

\section{3 \\ Foam Sweep Efficiency}

Foams that are stable, maintaining their structure and resisting collapse into a continuous separated gas and liquid phases, are beneficial for improving gas sweep efficiency. Moving bubbles and bubble interfacial area rearrangement are the first mechanisms by which foam affects gas mobility [12]. At low bubble velocities, the pressure drop to drive a bubble at a constant velocity surpasses that of an equal volume of liquid, hence increasing the effective viscosity of the gas phase. Furthermore, surfactant migration from the front to the back of a moving bubble causes a surface-tension gradient, which slows bubble motion and thus, raises effective viscosity [13].

The entrapment of the gas phase is the second mechanism that reduces gas mobility. The fraction of trapped gas in sandstone containing foam that is not flowing is estimated to be between 0.85 and 0.99 based on experimental data [12]. Pressure gradient, pore geometry, and foam texture are the key aspects affecting bubble trapping.

Foam tends to flow into zones with high permeability and porosity. The wetting surfactant solution occupies the smallest pore channels. As a result, gas is trapped in the pores of intermediate size [14]. Capillary forces appear 
to be significant to immobilize a considerable percentage of gas in bubbles. As a result, the pore space that might otherwise transport gas is closed, and gas relative permeability is greatly reduced.

The low flow mobility that foam exhibits in porous media happens because of the discontinuous nature of the gas. Clearly, if the lamellae that keep the gas disconnected ruptures, the foam will be destroyed and no longer be an effective mobility-control agent.

Figure 1.4 summarizes the mechanisms that affect the apparent viscosity of foam. On top, the presence of liquid between gas bubbles is responsible for creating resistance against flow. In the middle image, the viscous and capillary forces cause deformation, that is as a function of the flow rate. And in the bottom image, the surface tension gradient is caused by the surfactant depleted from the front that accumulates on the back of the bubble [13].

SLUGS OF LIQUID

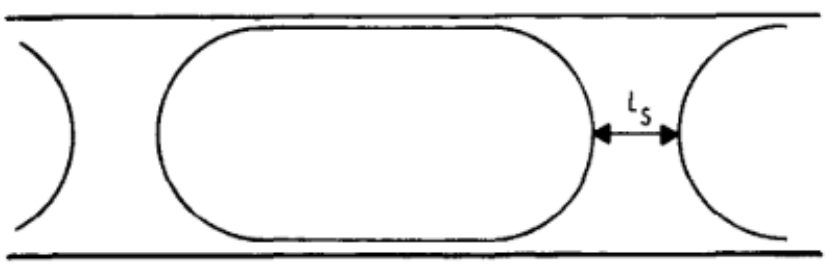

INTERFACE DEFORMATION

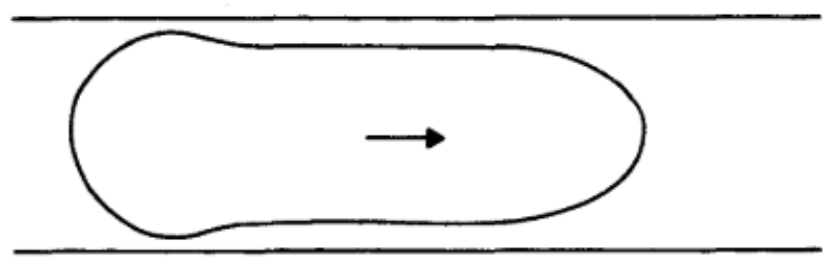

SURFACE TENSION GRADIENT

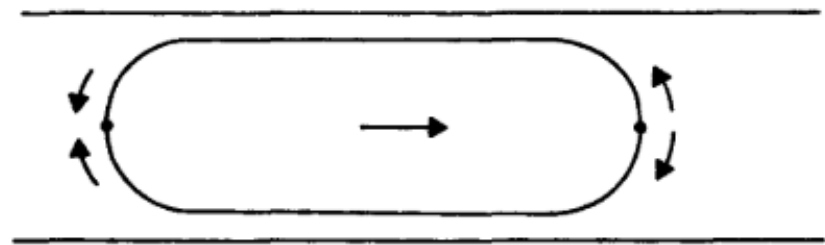

Figure 1.4: Mechanisms affecting apparent viscosity [13]

\section{4}

\section{Foam Generation in Oil Industry}

Pre-formed foam, co-injection foam, and surfactant-alternating-gas (SAG) injection are the types of EOR foam schemes employed in the field. Preformed foam is generated outside the porous medium. Foam can be formed at 
the surface, using a foam generator, during downward flow through the tubing and in the perforations, just before entering into the formation. Co-injection foam is formed in situ during co-injection of surfactant solution and gas. During co-injection, the surfactant can be injected continually or along with the water. The third foam scheme, SAG foam, is generated by alternate injection of surfactant solution and gas. During SAG injection, the foam is generated in the reservoir during drainage of the surfactant solution by gas [15].

Three criteria are critical when deciding between foam placement strategies (SAG, co-injection, or pre-formed foam): reservoir pressure, permeability, and the expected duration of surfactant injection (Figure 1.5). The co-injection foam is effective for low pressure and high permeability, and can be used for long-term injection. SAG injection at medium or even low surfactant concentration can be employed in high pressure and low permeability reservoirs.

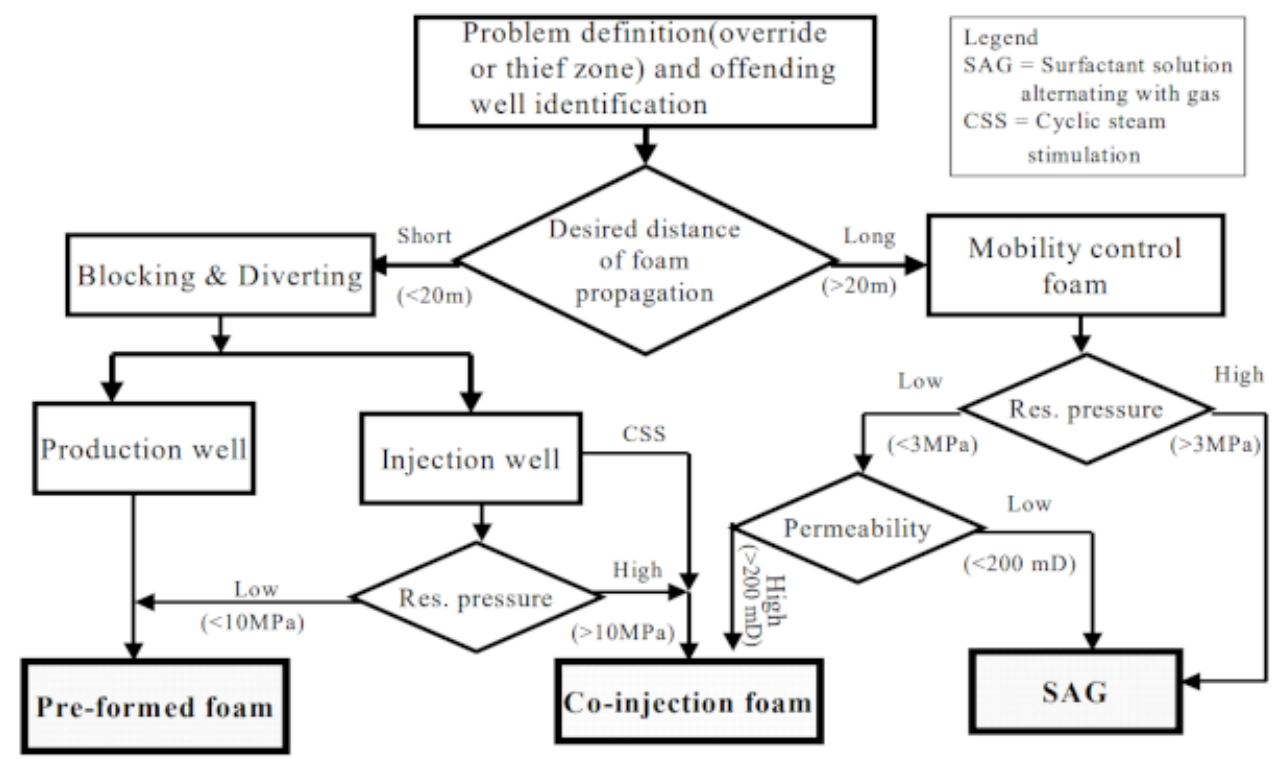

Figure 1.5: Foam placement strategies in EOR projects [15]

The behavior of foam in the first sections of the porous media is the fundamental difference between pre-formed foam and other types of foam. Commonly associated with high values of mobility reduction factor, pre-formed foam and co-injection sometimes cause excessive injectivity reduction. The resistance factor of pre-formed foam is higher, and it may even completely block the porous medium. The resistance factor of SAG foam is lower and it can not completely block the porous medium, thus presenting advantages over other methods [15]. 
Regardless of whether bubbles are formed externally or in situ, the porous media molds and reshapes them.

\section{5}

\section{Surfactant-alternating-gas injection}

Surfactant-alternating-gas injection, also known as SAG, is an enhanced oil recovery method in which alternated slugs of surfactant solution and gas are injected into a reservoir. The fluids mix inside the reservoir, generating foam that reduces gas mobility, thus improving the sweep efficiency. Surfactant also enhances recovery by lowering interfacial tension between the reservoir oil and the injection phases, leading to lower residual oil saturation.

SAG is operationally similar to WAG (water-alternating-gas) and has several advantages over continuous foam injection methods. SAG helps to reduce corrosion by reducing contact between gas and water in injection facilities. SAG's alternating imbibition/drainage cycles contribute to form foam in the reservoir. At the displacement front, SAG has a high injectivity and limited mobility.

Parameters like surfactant composition, gas/liquid ratio and flow rates define the success of this method. In Figure 1.6, it is possible to compare the displacing front for gas injection, water-alternating-gas, in this case $\mathrm{CO}_{2}$ and surfactant-alternating-gas.
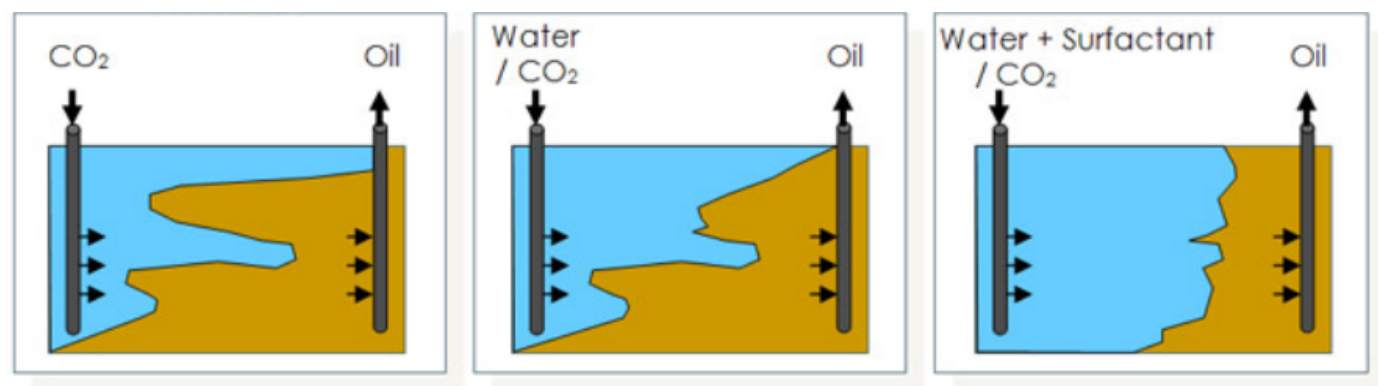

Figure 1.6: Displacing front for gas, WAG and SAG. Adapted from [16]

Most studies using micromodels aim to understand the dynamics of preformed foam flowing through a porous medium or foam formed during coinjection of surfactant solution and gas. Phenomena related to foam formation, destruction, stability, and increase in flow resistance were studied.

In the core-flooding experiments, the impact of foam injection or foam formation during the SAG method on oil recovery in representative samples of a reservoir was studied. However, it is not possible to visualize the phenomena that occur inside the rock. 
This work targets the visualization of foam formation and the mechanisms involved in its formation and destruction when it occurs inside a porous media micromodel. The analysis aims to understand the effect of surfactant concentration, below and above the CMC (critical micelle concentration), on foam formation and oil displacement in the SAG method during Enhanced Oil Recovery.

\section{6}

\section{Outline}

This thesis topics are divided in six chapters.

In this chapter, Chapter 1, a brief introduction was addressed with the main concepts that are used during the thesis.

Chapter 2 discusses foam structure, foam formation and destruction mechanisms, besides foam transport inside porous media and the parameters to assure its stability. The discussion is based on the literature.

Chapter 3 describes the materials used in the experimental analyses, including fluids and the glass micromodel.

Foam formation studies are presented in Chapter 4. This comprises the two-phase flow experiments procedure, the image analysis technique and the main results.

Chapter 5 outlines the surfactant-alternating-gas experiments, detailing the setup used, image analysis procedures and results.

Finally, conclusions and future steps are presented in Chapter 6. 


\section{2}

\section{Literature review and thesis goals}

\section{1}

\section{Foam}

Foam is a two-phase system in which gas is enclosed by surfactantcontaining liquid films, called lamellae. It may be classified according to the liquid fraction: a dry foam that has low liquid fraction, consists basically of thin films. As the fraction of liquid increases, foam reaches the other limit, called wet foam. When foam loses its rigidity, it is a bubbly liquid. The wetting phase, in this case, the surfactant solution, remains continuous even at very low saturations.

As shown in Figure 2.1, the liquid fraction $(\phi)$ ranges from less than one percent (dry foam) to more than 10\% (wet foam). When the liquid fractions are low, the bubbles deform into polyhedral with quasi-flat faces and curved faces at the edges. For a critical value $\phi_{c}$ (approximately $35 \%$ for disordered foams and $25 \%$ for ordered foams) the bubbles are spherical and move freely, resulting in bubbly liquid in which the foam behavior is no longer solid-like (finite shear modulus G) and becomes liquid-like [17].

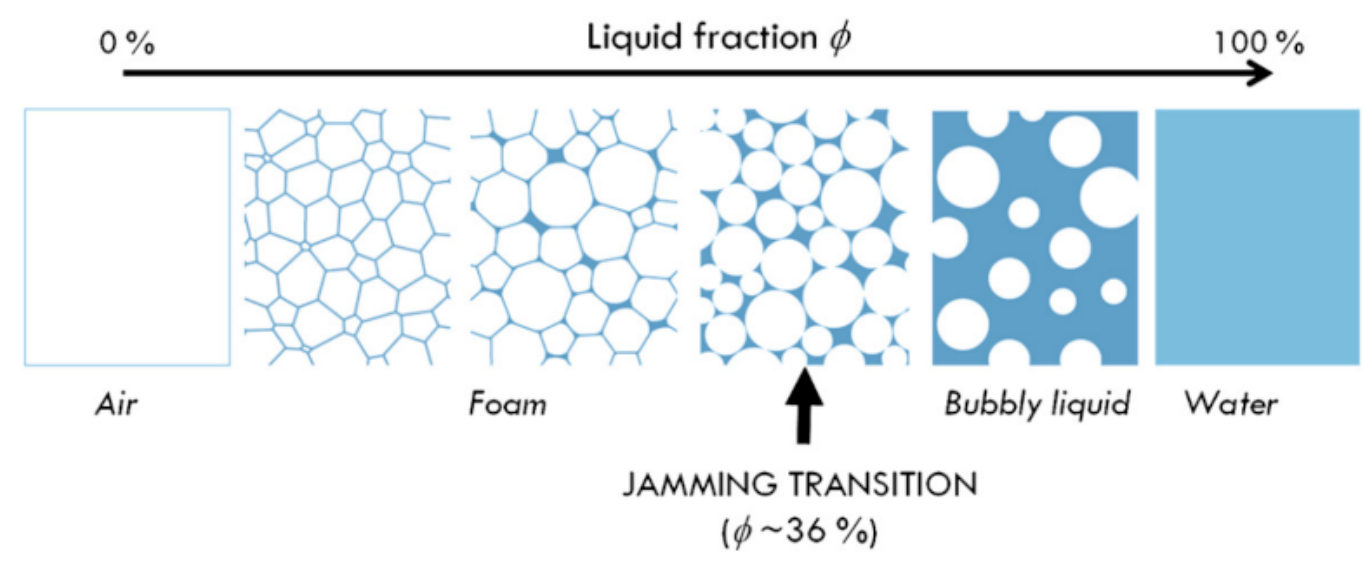

Figure 2.1: Foam aspects for different liquid fractions [17]

A foam is usually disordered, which relates to the foam's bubbles, that vary widely in size. Ordered foams are a topic of theoretical investigation since they do not occur in real life. In dry foam (Figure 2.2), liquid films (lamellae) 
separate the sides of the bubbles, and Plateau borders (the channels between bubbles) and nodes are present at the corners. Surfactant monolayers stabilize the liquid films, the polar heads are represented by circles and the hydrophobic chain is represented by the tail. Surfactant molecules may also be present in the bulk, as illustrated in Figure 2.2.

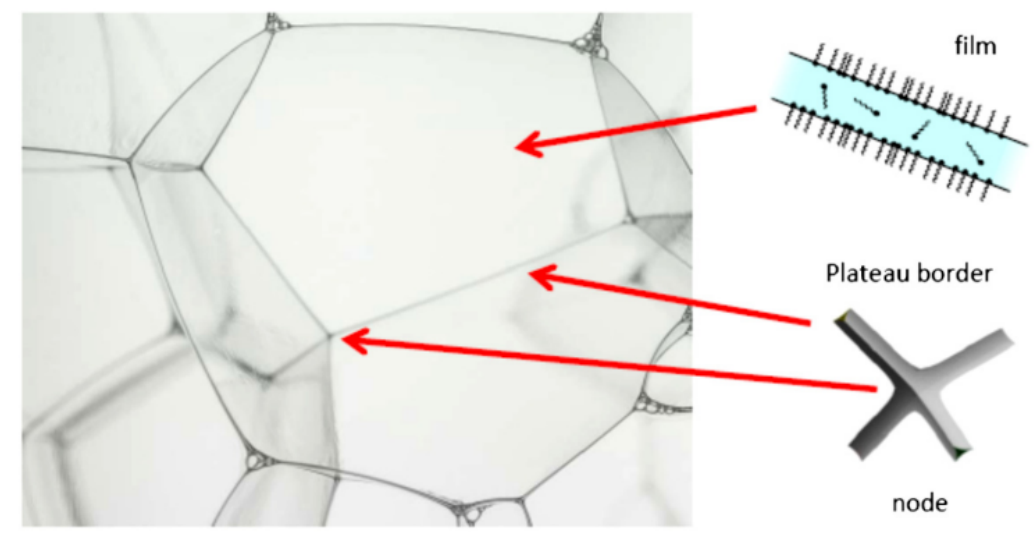

Figure 2.2: Dry foam [17]

Plateau borders coincide with foam films in a two-dimensional foam. Bubbles/cells, films/Plateau borders and Plateau junctions are the three elements that compose a foam. These parts correspond to faces, edges, and vertices in mesh topology, respectively.

In 1993, Kovscek and Radke [14] compiled all the information available on foam generation, destruction and transport mechanisms. Also, they proposed a one-dimensional mechanistic model for transient foam displacement based on bubble size evolution. Using a sandstone core as the porous medium and nitrogen as the gas phase, co-injection experiments were performed on a wide range of gas and liquid flows to understand and quantify foam flow phenomena at the pore scale.

\section{2}

\section{Foam generation}

It is important to understand the pore-level lamellae creation and destruction mechanisms to comprehend foam formation and flow in porous media. There are four mechanisms for lamellae creation that have been proposed in the literature $([18] ;[19])$.

\subsubsection{1}

\section{Leave behind}

When stabilized liquid films are created in the throats of the pore space as gas invades adjacent pore bodies through other throats, the formation 
mechanism is called Leave behind, illustrated in Figure 2.3. For the lamella to be stable, a surfactant solution must be present. Depending on the geometry of the porous medium, liquid films can break if the surfactant is not present. It is known as an ineffective foaming method, however, a large number of lamellae can be created during this process. However, if it is the only mechanism for creating lamellae, the gas will always be connected via a continuous gas path [18].
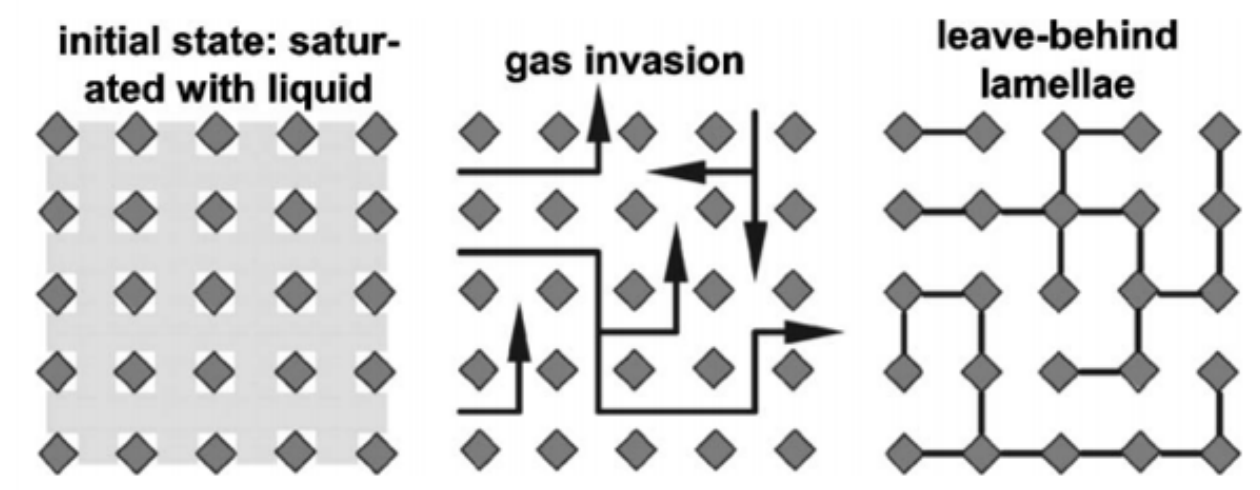

Figure 2.3: Leave behind [18]

\subsubsection{2}

\section{Lamella division}

When two or more lamellae are created from a single one, it is called Lamella division mechanism. If a mobilized lamella passes a pore body, with more than one pore throat unoccupied by liquid or another lamella, it must break or span through the two throats, as shown in Figure 2.4. Lamella division can result in a large region of the pore space being filled with lamellae, starting with only a small number of moving lamellae [18].

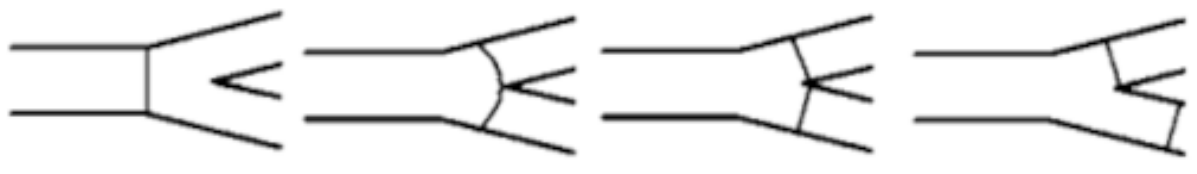

Figure 2.4: Lamella division [18]

\subsubsection{3}

\section{Snap-off}

In the mechanism known as Snap-off, the lamellae are created in the throat of the pores that are occupied by gas if the local capillary pressure 
drops to about half of the capillary entry pressure of the throat (Figure 2.5). This value depends on the geometry of the throat and the wettability of the porous medium [18].

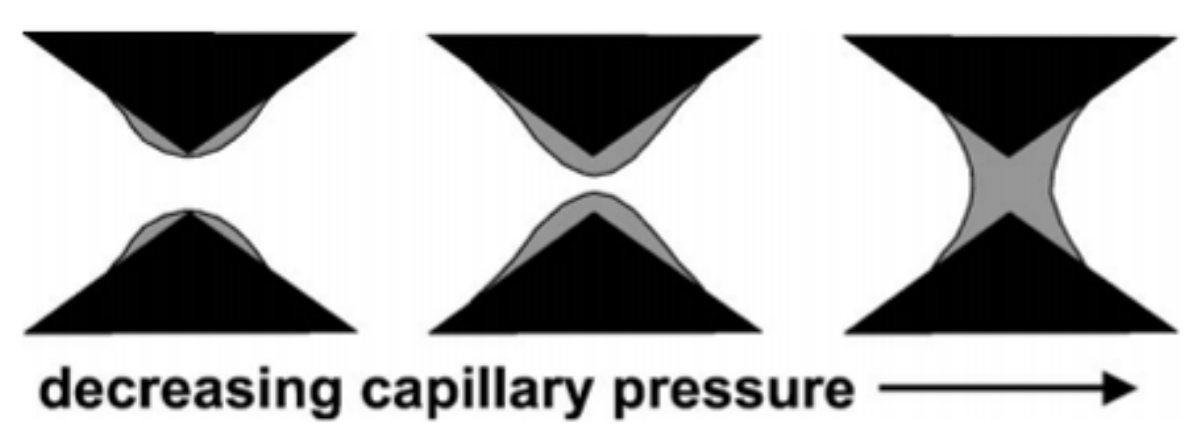

Figure 2.5: Snap-off mechanism. Black is the pore-throat wall, gray is the water and white is the gas. [18]

When gas invades a large, fluid-filled pore body through a narrow pore throat, the liquid that drains from the pore body can return to the throat driven by capillary force and disconnect the gas phase, as illustrated in Figure 2.6. The mechanism is known as Roof Snap-off after Roof's study of oil trapping in waterflooding [20].

In the Roof Snap-off, the size of the pore body in relation to the throat is important, because it is its radius that controls the local capillary pressure at the moment when the gas fills the pore body. Some pores become "germination sites" for the creation of lamellae, but only when the gas invades a liquid-filled pore body. In all other Snap-off mechanisms, the Snap-off depends only on the radius of the throat and the local capillary pressure, that fluctuates according to the movement of the lamellae and is lower at the back of long bubbles than at the front edge [20].
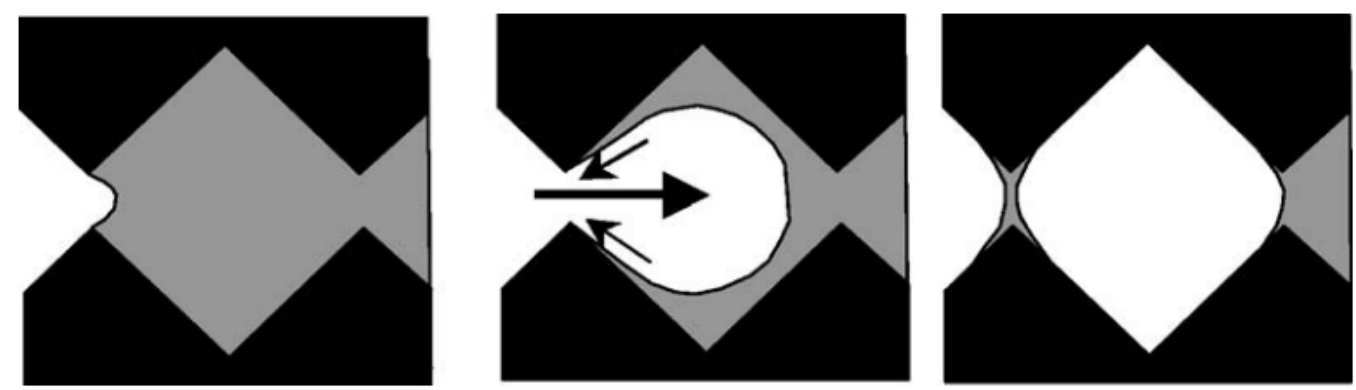

Figure 2.6: Roof Snap-off as gas invades a pore body. Same color scheme as previous picture.[18] 


\subsubsection{4}

\section{Pinch-off}

Pinch-off mechanism is observed in regions where bubbles can deform easily and can generate bubbles smaller than the characteristic dimension of the pore, which cannot be done in any of the methods explained previously.

This mechanism can be explained through Figure 2.7. A pink colored bubble pinned a green one against a post and stretches it until two points at the green bubble interface meets and the bubble breaks into two separate bubbles. The compression induced by neighbors differs from the Lamella division in its driving force and in the size of the bubbles it produces. The driving force for neighbor-induced compression is the tensile stress that arises from the change in the morphology of the individual bubbles, while the driving force for Lamella division is the pressure difference across the liquid film, and no significant deformation of the bubble is observed [19].

Bubbles enter pore throat

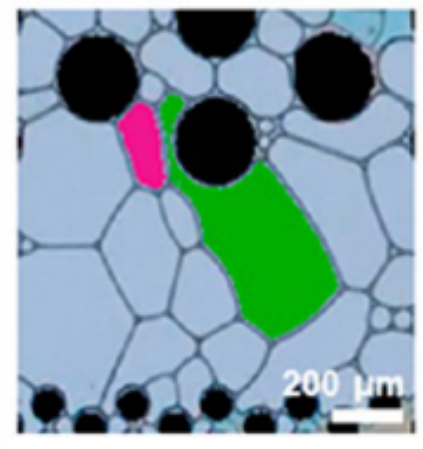

Bubble squeezed

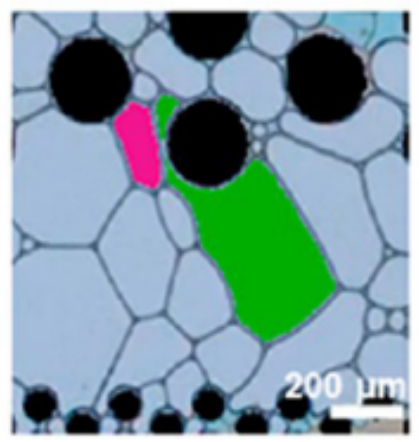

Bubble pinched add lamellae

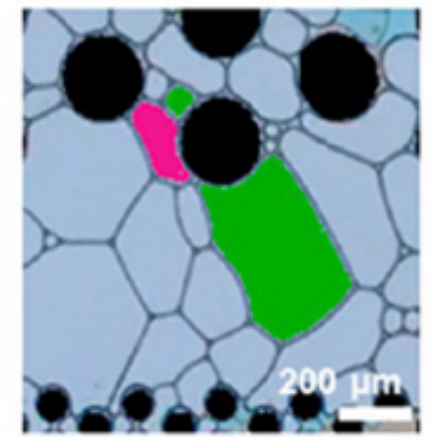

Figure 2.7: Pinch-off mechanism: the pink bubble pinches the green bubble against the post, forming a new bubble [19]

This mechanism can be divided into "neighbor-wall pinch-off" and "neighbor-neighbor pinch-off". When a bubble is pinched between the surfaces of a neighboring bubble and the curved wall of the constriction, it is called a "neighbor-wall pinch-off". And when a bubble is pinched off between two adjacent neighboring bubbles, this is known as "neighbor-neighbor pinch-off" [21].

\subsection{1}

\section{Regimes of foam flow}

Because of the moderate reduction in gas mobility, coarsely textured foam (small number of lamellae with large bubble size) is often referred to as weak foam. Finely textured foam (large number of lamellae with small bubble size), on the other hand, is referred to as strong foam since it significantly restricts gas mobility. 
The two types of foams in porous media are depicted schematically in Figure 2.8. In the presence of foam in porous media, gas mobility is typically reduced by 10 to 100 times for weak foam and more than 10,000 times for strong foam. In other words, foam texture has a significant impact on the rheological properties of foam in porous media [22, 23].

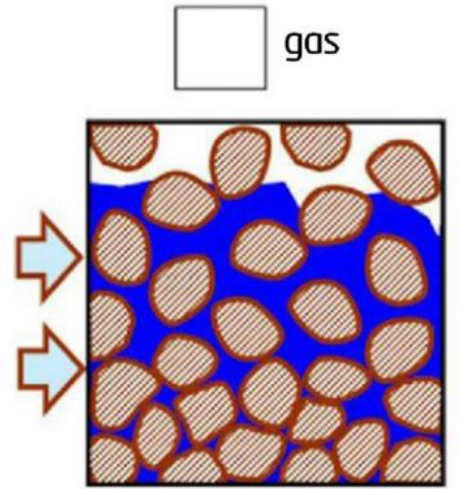

(A) Gas-liquid (No Foam)

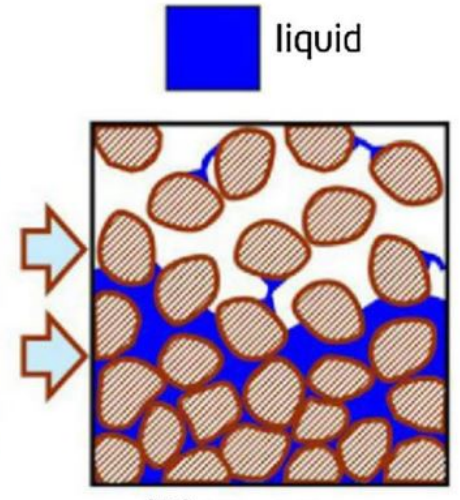

(B) Weak Foam

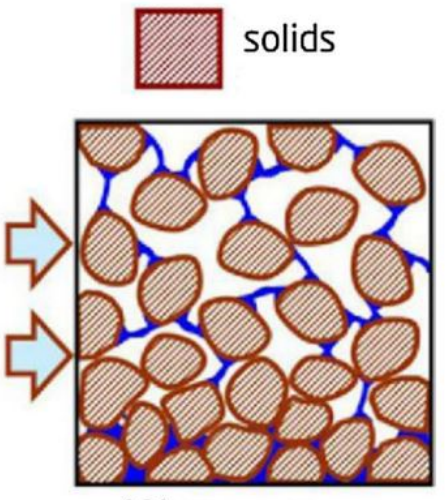

(C) Strong Foam

Figure 2.8: Concepts of weak and strong foams within porous media [24]

\subsection{2}

\section{Critical pressure gradient and rate of foam generation}

In addition to the foam formation mechanisms, ideal pressure conditions are required to generate strong foam. Foam generation refers to the transition from no foam to strong foam, as well as the transition from weak continuous-gas foam to strong discontinuous-gas foam. Leave-behind process produces weak foam, but strong foam is normally created by a combination of all methods.

In 1988, Ransohoff and Radke [25] performed experiments with monodisperse bead packs to understand the pore-level mechanism of foam generation. They were the first to consider the effects of rates and pressures when calculating a critical capillary number for foam formation. They established that above a critical velocity, sufficient for the Snap-off mechanism to occur, strong foam is formed. A simple model based on the concept of a germination site was proposed to predict the onset of Snap-off.

Another theory was proposed by Rossen and Gauglitz [26] stating that lamella mobilization and not only Snap-off were responsible for foam generation. The surface tension forces that hold a lamella in place must be overcome by a sufficiently high velocity or pressure gradient for the lamella to be mobilized.

When the pressure difference across the lamella exceeds a particular "yield" pressure, a lamella that is blocking a pore throat becomes mobile, 
this value can be estimated as

$$
\Delta p_{y 1} \approx \sigma / \sqrt{k / \varphi}
$$

where $y$ stands for yield, 1 for a single lamella, $\sigma$ is interfacial tension, $k$ is permeability and $\varphi$ is porosity. The same idea applies for a series of lamellae:

$$
\Delta p_{y}=n_{L} \Delta p_{y 1} \approx n_{L}(\sigma / \sqrt{k / \varphi})
$$

where $n_{L}$ is the number of lamellae over the system [27].

Lamella division and/or repeated Snap-off at the unoccupied pore constriction can multiply the number of lamellae once they have been mobilized. The existence of a critical pressure gradient for foam mobilization that is inversely related to permeability has been proposed by steady-state theories and experiments in the literature [27].

During surfactant solution and gas flow through porous media, a critical pressure gradient must be exceeded to form strong foam ([27]; [28]). Gas may flow as a continuous phase below this pressure gradient. And above this pressure, stationary bubbles are mobilized and flow in bubble trains. A bubble that is flowing can be divided into two bubbles. If the critical pressure gradient required for strong foam is established, experiments can be carried out at a high enough flow rate or pressure drop to exceed this pressure.

Foam generation occurs above the minimum pressure gradient as sketched in Figure 2.9. In the coarse or weak foam regime, there's limited lamella creation. Both lamellae production and destruction start once strong foam regime is achieved.

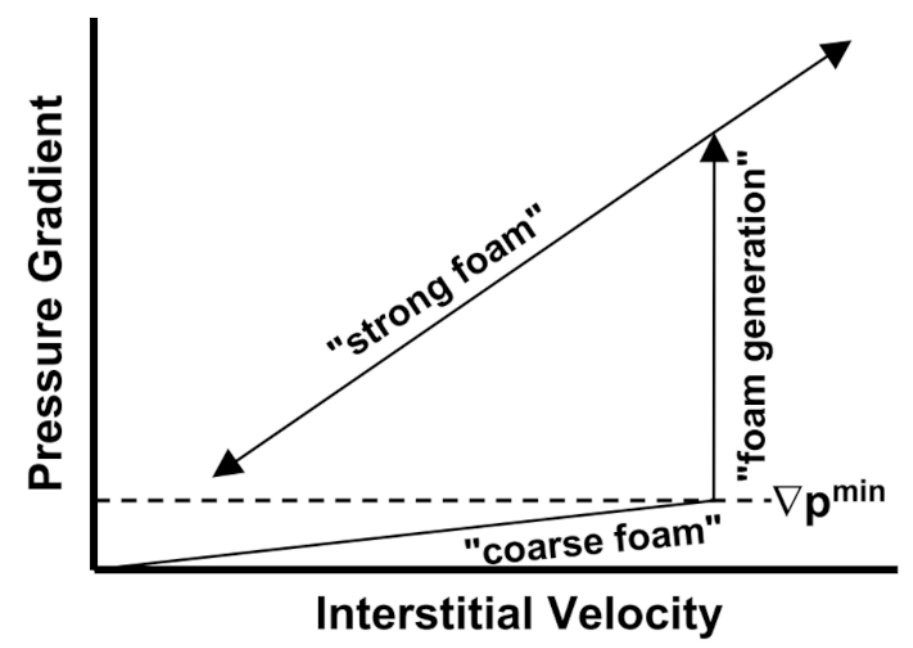

Figure 2.9: Pressure gradient as a function of interstitial velocity [28] 
Tanzil et al. [27] concluded that a critical pressure drops scales with $1 / \sqrt{k}$ rather than $1 / k$ predicted by Gauglitz [28]. The difference between the values found can be explained by the fact that they used different surfactant solutions. In addition to having performed tests with very different experimental procedures, Tanzil performed transient analysis, while Gauglitz performed tests in steady state conditions. The conclusion is that every system has its own minimum pressure gradient that is dictated by the surfactant formulation, porous media and experimental procedure.

Another important advance is understanding that once the minimum pressure is achieved, the rate of foam generation in porous media is highly influenced by the surfactant concentration. This is most likely related to the rate at which lamellae are created and destroyed. As the surfactant concentration rises, the rate of foam generation rises as well [29].

\subsection{3}

\section{Lamella-destruction mechanism}

\subsubsection{1}

\section{Coarsening}

In porous media, coarsening or Ostwald ripening happens due to diffusion, which is more important in the trapped fraction of foam. It was first investigated by Wilhelm Ostwald around 1900. Gas diffusion is driven by pressure differences between adjacent cells of unequal sizes, so large droplets or bubbles grow at the expense of smaller ones. The mass-transfer rate of gas through lamellae depends mainly on the gas and surfactant type [30].

The coarsening phenomenon is illustrated in Figure 2.10, in which the gas inside the smaller bubble (gray) diffuses to the neighboring bubbles.

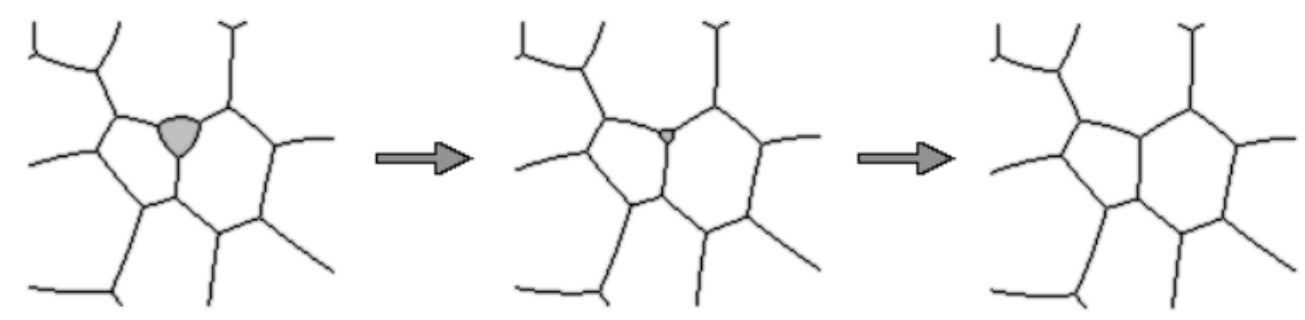

Figure 2.10: Coarsening [31]

Regarding foam destruction mechanisms, in 2018, Jones et al. [32] studied the effect of coarsening on a porous medium using microfluidics devices and sandstone cores. One example of the microfluidic experiment is shown in Figure 2.11. Surfactant solution and nitrogen were co-injected into the micromodel 
until steady state flow was achieved and no major foam evolution was observed. The flow was stopped and the micromodel sealed to visualize the coarsening phenomenon. After 2000 seconds (Figure 2.11 (b)), the majority of the lamellae were in the pore throats, regions with minimum energy configurations. This stable energy configuration is detailed in Figure 2.12 and happens due to diffusion between the bubbles.
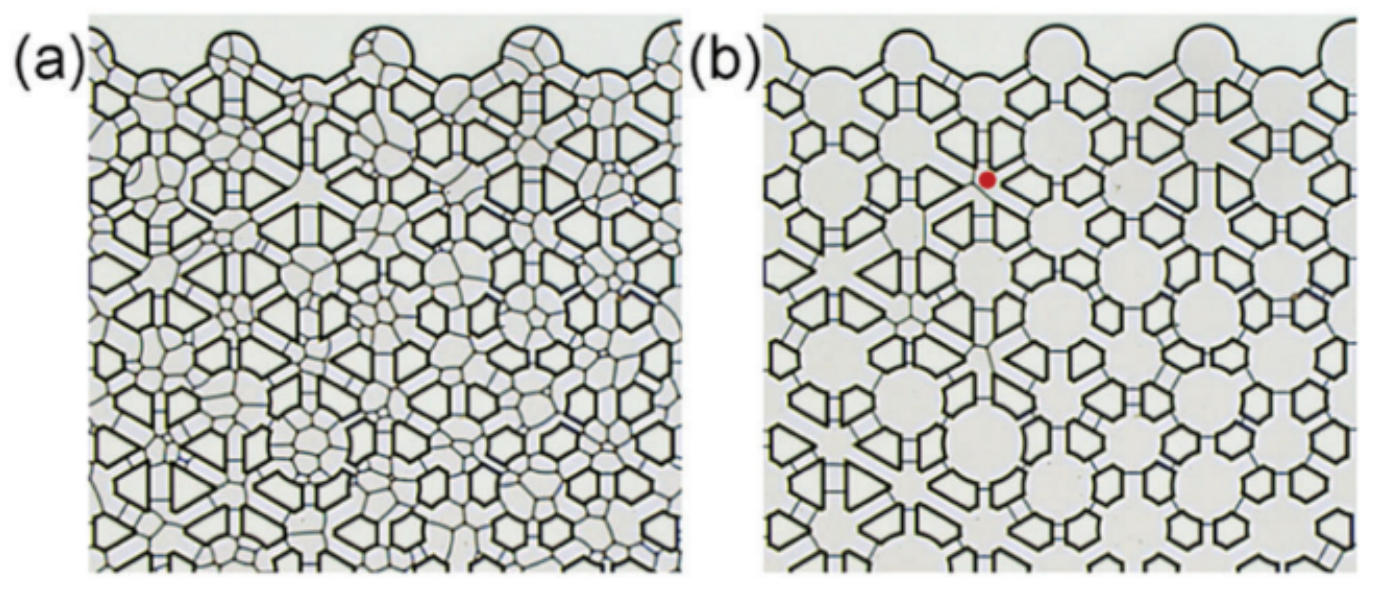

Figure 2.11: Foam coarsening mechanism can be observed in a glass micromodel. In (a) at the beginning of the experiment $t=0$ and in (b) after 2000 seconds [32]

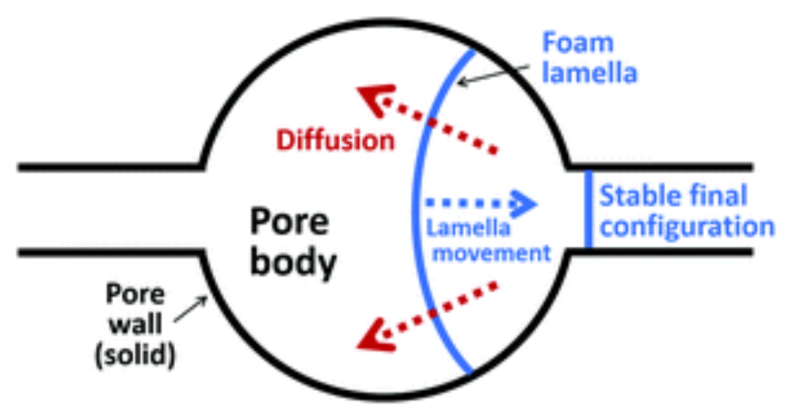

Figure 2.12: Minimum-energy configuration of the lamella in the pore throat $[32]$

\subsubsection{2}

\section{Capillary suction}

Surfactant composition has a significant impact on capillary-suction coalescence. Thin lamellae are not thermodynamically stable. Excess normal forces inside the films, resulting from long-range inter-molecular interactions, are responsible for their existence.

Figure 2.13 explains the suction mechanism. The liquid pressure in the Plateau border $\left(P_{w}\right)$ is lower than in the lamella $\left(P_{w L}\right)$ due to the curvature 
of the gas-liquid interface. The resulting suction drives a flux of liquid from the lamella into the Plateau border. If there is nothing to oppose this suction force, then the lamella thins rapidly until it becomes unstable and ruptures. The other forces illustrated are the capillary pressure $\left(P_{c}\right)$ and the pressure in the gas phase $\left(P_{g}\right)$.

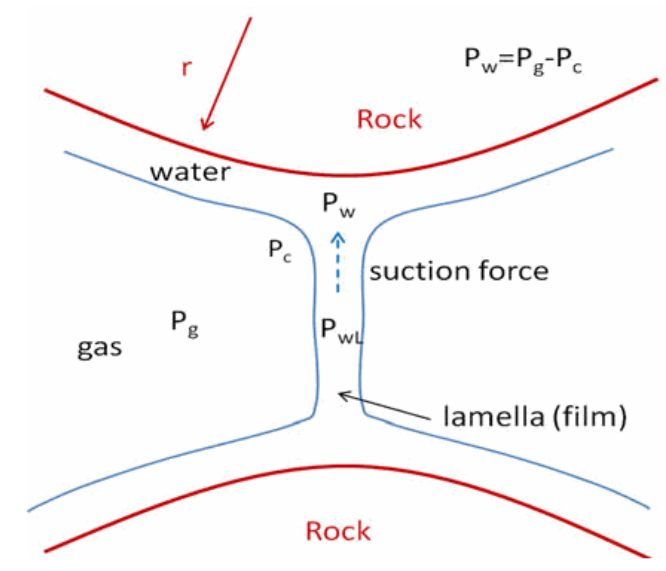

Figure 2.13: Scheme of capillary suction inducing film thinning [33]

The DLVO theory was named after Boris Derjaguin and Lev Landau, Evert Verwey and Theodoor Overbeek ([34];[35]) research explaining the aqueous dispersion aggregation and describing the forces between charged surfaces interacting through a liquid medium. It combines the effects of the van der Waals attraction with the electrostatic repulsion because of the so-called double layer of counter-ions.

Derjaguin and colleagues [36] proposed this concept in terms of a film disjoining pressure, $\Pi$, that is a function of film thickness. Net repulsive film forces are represented by positive $\Pi$ values, whereas net attractive film forces are represented by negative $\Pi$ values. The excess repulsive forces are caused by ionic surfactant adsorption on each gas/liquid surface of the film. Through the overlap of their doublelayer ionic clouds, the equally charged surfaces repel each other. Protrusion and/or hydration forces cause a very steep repulsion at small film thicknesses. These two stabilizing forces are affected by surfactant concentration and structure, as well as the aqueous solution's ionic content. Surfactant formulation has a role in the development of effective foamers. Furthermore, the film is destabilized by attractive van der Waals forces. An S-shaped disjoining pressure isotherm results from a complex interaction between these three forces.

Figure 2.14 shows a typical disjoining pressure isotherm. The three primary components of the disjoining pressure are the electrostatic repulsion 
$\Pi_{e l}$, the van der Waals attraction $\Pi_{v w}$, and the steric/hydration forces $\Pi_{s h}$.

$$
\Pi=\Pi_{e l}+\Pi_{v w}+\Pi_{s h}
$$

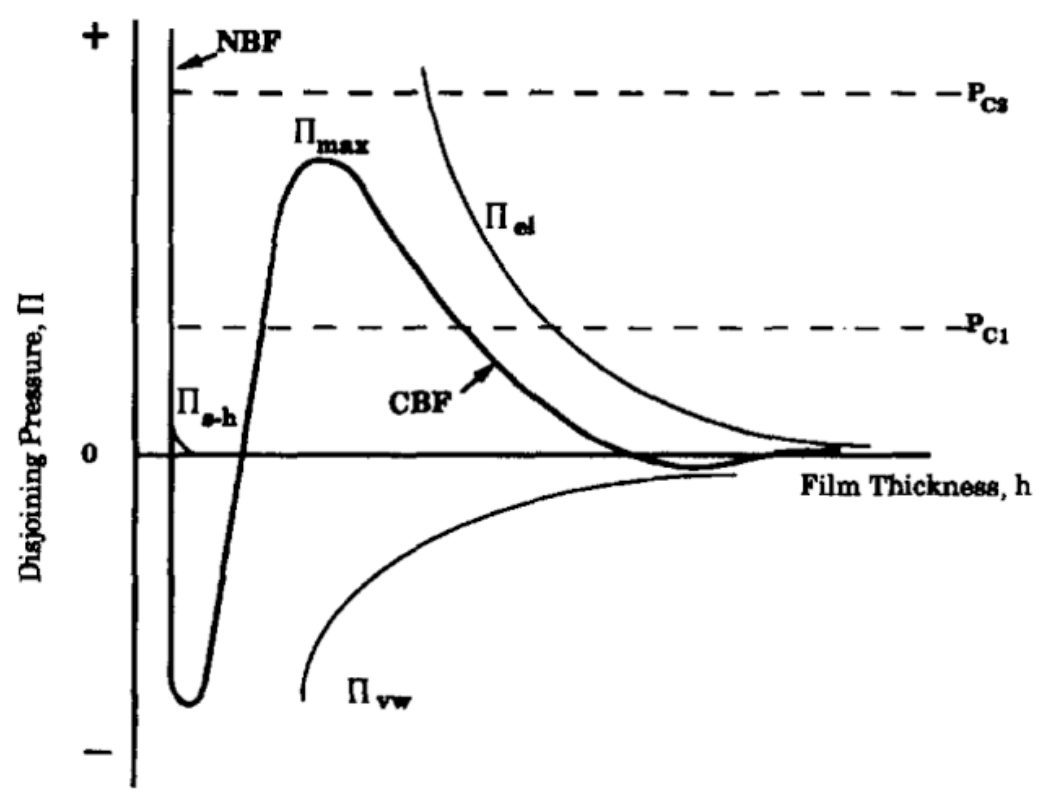

Figure 2.14: Disjoining pressure isotherm [37]

As the capillary pressure in the porous medium rises during drainage, the film thickness $(h)$ decreases from infinity and the first maximum $\Pi_{\max }$ arises. It happens due to the repulsive overlap of the electrostatic diffuse double layers overpowering van der Waals attraction. These films are known as common black films (CBF) or common thin films. The thickness decreases further and van der Waals attraction becomes important, so the slope of the isotherm changes. When steric/hydration forces become strong relative to the attractive branch, a primary minimum in the isotherm occurs. The steric/hydration forces produce an extremely steep repulsive branch when the film thickness decreases past the minimum. Newton black films are those made on the deepest branch (NBF). The thin film is thermodynamically unstable and equilibrium films cannot occur where the isotherm has a positive slope [37].

The dotted horizontal lines in the plot represent the capillary pressure applied on the liquid films. Both flowing and stationary foam films thin (or thicken) until achieving an equilibrium thickness in accordance with the augmented Young-Laplace relation (Equation 2-4), at a given capillary pressure.

$$
P_{c}=2 \sigma C_{m}+\Pi(h),
$$


where $P_{c}$ is the local capillary pressure, $\sigma$ is the bulk surface tension of the surfactant solution and $C_{m}$ is the mean curvature of a lamella [37]. The film curvature is determined by the specific location within the pore structure defined by a $90^{\circ}$ contact angle with the pore wall and the capillary pressure is determined by the wetting liquid saturation.

When the liquid film is thick enough, the disjoining pressure approaches zero and the classic Young-Laplace equation is restored. At high capillary pressures caused by low permeability and low liquid fractions, the lamella eventually ruptures. This pressure is referred to as critical capillary pressure for rupture or capillary suction pressure. Wetting liquid saturation, permeability and surface tension are the parameters controlling this pressure.

In flowing foam bubbles, the behavior is more complicated than static ones. For a specific surfactant system, a significant foam coalescence at a particular capillary pressure termed the limiting capillary pressure, $P_{c}^{*}$ can be observed. Above $P_{c}^{*}$, foam films are unstable, coalescence is considerable, and high gas mobility emerges. Below $P_{c}^{*}$, coalescence is significantly less and gas mobility is low. Surfactant structure and concentration, electrolyte concentration, gas flow rate, and permeability all affect the limiting capillary pressure [14].

Aronson [37] measured the disjoining pressure isotherms of sodium dodecyl sulfate (SDS) solution and the pressure drop obtained by injecting the same solution in a sandpack to investigate the relationship between the critical and limiting capillary pressures. Their findings revealed that the solution with the highest disjoining pressure have the greatest pressure drop in the porous media, resulting in the greatest flow resistance. This limiting capillary pressure corresponds closely to the maximum or rupture value on the disjoining-pressure curve. However, the experiments carried out by Aronson consisted of the coinjection of a surfactant solution and gas, in this case the liquid fraction does not decrease with time, as it happens in the experiments performed in this thesis.

Foam formation is a complex phenomenon, it happens only above a minimum flow rate, above a minimum pressure gradient, and below a critical capillary pressure.

\section{3}

\section{Foam drainage and stability}

One of the most distinguishing features of aqueous foams is their irreversible evolution over time: foams drain, coarsen, and eventually collapse as the films between bubbles break. Understanding the behavior and stability of 
these thin liquid films is crucial to establishing the impact on flow in porous media.

With time, the liquid and the gas in the foam tend to separate due to gravity and capillarity. The liquid drainage leads to thinning of the films separating gas bubbles and to their eventual rupture.

The thinning and the lifetime of foams were investigated by Velikov et al. in 1997 [38]. They studied the effect of surfactant concentration on the kinetic stability of foam and compared it with hydrodynamic theories of film drainage. They concluded that the time of hydrodynamic drainage of the films increased linearly as the logarithm of the surfactant concentration. The final (black) films, as well as the entire system, will most likely be stable if the surfactant concentration is above the CMC. However, increasing the surfactant concentration even further could increase the foam's hydrodynamic stability. As a result, homogenization with a surfactant concentration slightly above the $\mathrm{CMC}$ is the minimum required condition for obtaining a practically stable foam, but the hydrodynamic stability of the system can be increased with further increases in concentration. One example of this behavior is shown in Figure 2.15 [38]. For this case, the CMC is $0.001 \mathrm{M}$, the film lifetime continues to increase as the surfactant concentration increases above the CMC.

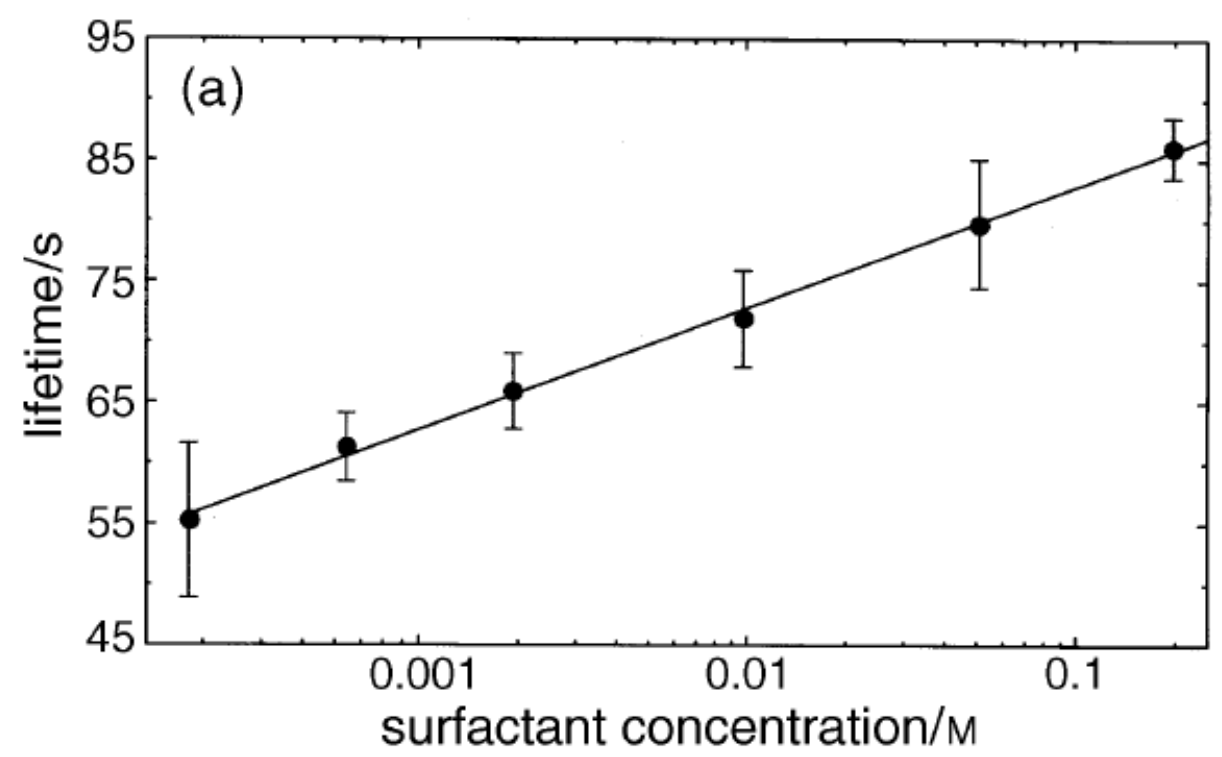

Figure 2.15: Hydrodynamic lifetime of foam films versus surfactant concentration [38]

The stability of lamellae against rupture decreases as they become thinner. The foam destabilizes and collapses. The only way to get a persistent foam is for the drainage to result in the creation of a black equilibrium film, 
rather than destabilizing the film. They are called black films because they are too thin to reflect incident light [39].

According to Hédreul and Frens [39], there are two types of black films. When the draining lamella reaches an equilibrium thickness, the thicker or common black film (CBF) forms. The capillary pressure and the van der Waals attraction in the film are compensated by repulsion between adsorbed surface layers at this film thickness. And the Newton black film (NBF), which is a thinner type of equilibrium film, which reflects the equilibrium in the primary minimum of the potential energy of contact.

Both the NBF and the $\mathrm{CBF}$ are equilibrium films that reflect potential energy minima. However, the NBF is more structured and elastic than the CBF, has lower free energy and a lower surface tension.

A thin liquid film is not an isolated object, it is connected to other lamellae by Plateau borders. The thickness fluctuations and capillary suction act with different forces on thicker and thinner lamellae.

A thickness fluctuation may begin to grow at a Plateau Border, helped by the Laplace underpressure, or at the middle of the film. Drainage will continue after that, but it will finally come to a stop at the CBF equilibrium film thickness. If the thickness variation continues to develop, the film will either break or end up as a Newton Black Film, notwithstanding the energy barrier.

The transition from $\mathrm{CBF}$ to NBF is only possible at the higher surfactant concentrations because NBF formation requires interfaces with high surfactant coverage [40].

\section{4}

\section{Flow in porous media}

Gas can be present in different ways in a porous medium, trapped or flowing as a continuous or discontinuous phase, as sketched in Figure 2.16. The gas phase can be discontinuous and separated by liquid films, such that no continuous path is formed percolating the porous media. The gas can also be encapsulated in bubbles stabilized by surfactants. When the gas flows continuously, it is not intercepted by lamellae at macroscopic distances, much larger than the dimensions of the pore.

If the wetting phase saturation and flow rate are high enough for foam generation, foam can occur while gas and surfactant solution are injected. When the wetting phase saturation is low, the rate of lamellae generation can be exceeded by the rate of rupture, resulting in continuous gas paths [41]. 


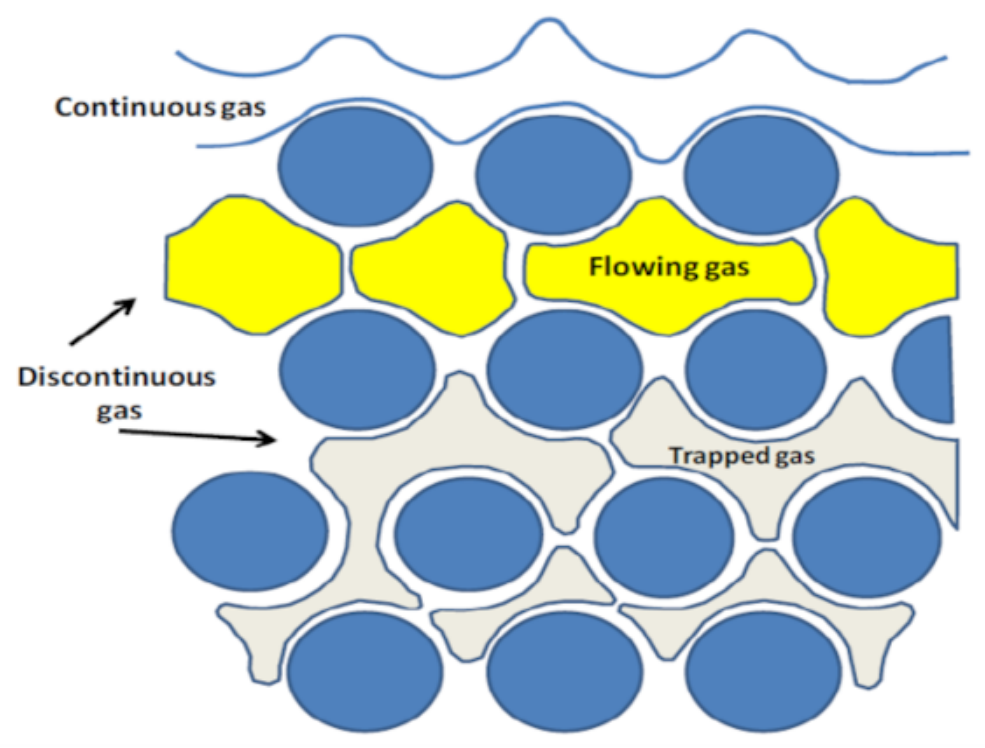

Figure 2.16: Different forms that gas can take in porous media [41]

The flow behavior is different depending on the liquid fraction. If weak foam is present with no moving lamellae, the gas is trapped in situ in the pore space blocking gas paths and reducing gas relative permeability. In this case, the rest of the gas flows as a continuous phase.

For strong foam, the flow pattern is different. The liquid films make the flowing gas discontinuous, bubble trains appear and face much higher resistance than in continuous gas flow. The apparent viscosity of the discontinuous gas is much larger than in the continuous gas flow. The combined effect of gas relative permeability reduction and apparent gas viscosity increase impact and considerably increase the mobility reduction effect of foam [41].

The ability of foam to decrease gas mobility is highly influenced by its texture, which refers to the size of the bubbles or the number of lamellae per unit volume. Structural changes happen during the equilibration and evolution of a dry foam, lamellae are created and destroyed and its texture has important effects on flow phenomena in porous media.

The injection of a water-soluble surface-active agent with a foamproducing tendency to improve the efficiency of gas-drive secondary oil recovery process was for the first time cited in a patent in 1956. Boud and Holbrook [6] described the impact of adding a surface-active agent in floodwater to improve oil production by reducing the mobility of the gas phase and increasing the displacement capacity of the injected gas. To illustrate the advantages of producing foam within the rock they used a glass tube filled with sand to perform recovery experiments. 
Foam structure in porous media implies that gas mobility may be conceptually divided on two effects: gas relative permeability and viscosity.

In 1964, Bernard and Holm [42] remarked that the simultaneous flow of water and gas had been studied by several researchers, but that the foam properties were considerably different from those of its components. As an example, they cited its viscosity, that is greater than both of its components. They studied the stability of foam in a porous medium under different conditions, varying the foaming agent concentration, injection rate, pressure and porous medium properties. Using consolidated and unconsolidated sands they concluded that foam is exceedingly effective in reducing the permeability of porous media to gas. It causes also a selective plugging of high permeability channels in various displacement processes.

To understand the impact of drag and resistance to the flow of lamellae through pores and throats, Hirasaki and Lawson in 1985 [13] used smooth capillaries to measure and mathematically model both effective and apparent viscosity of foam. According to them, surfactants maintain a constant equilibrium tension around the bubble during the flow. The bubble front interface stretches toward the capillary wall during flow, whereas the posterior bubble interface contracts toward the capillary centerline. The surfactant depletes at the bubble front, resulting in a surface tension higher than the bulk equilibrium value, whereas the surfactant accumulates on the bubble's back, resulting in a surface tension lower than the equilibrium value. A surface-tension gradient appears, which is directed towards the bubble front and slows bubble movement.

The higher the value of the number of bubbles per unit volume, the finer the foam texture is, therefore, the lower the gas mobility. Also texture is a dependent variable and will depend on the mechanisms of foam generation and stability [13].

Also, concerning the apparent viscosity of foam, Falls et al. in 1989 [43] explained that during flow, when foam's texture is unaltered by the porous medium, trapped bubbles block most of the cross-sectional area, and moving lamellae flow through a single channel. The surrounding stationary lamellae moved back and forth as the lamellae traveled along this path, giving the impression that they were "breathing". The viscous resistance to lamellae flow, as well as neighboring stationary lamellae that fluctuate in reaction to pressure changes in the flowing portion of the foam, consume energy. This energy dissipation depends on the number of lamellae per unit length. If the number is large compared with the spacing of constrictions and there are many stationary lamellae, almost all the energy is lost. But when the bubble size 
becomes smaller and all lamellae flow, a higher fraction of the energy can assist the flow of others.

\section{5}

\section{Foam as an EOR method}

A study conducted by Conn et al. in 2014 [7] focused on visualizing pore scale phenomena during oil displacement with foam using a microfluidic device with permeability contrast. Foam was pre-generated and injected into a 2-D PDMS micromodel saturated with oil. As shown in Figure 2.17, the device has two stratified regions, one with high permeability and the other with low permeability, separated by a central channel. Prior to injection into porous media, foam was generated using a flow-focusing microfluidic device (Figure 2.17) that produced mono-disperse bubbles in series.
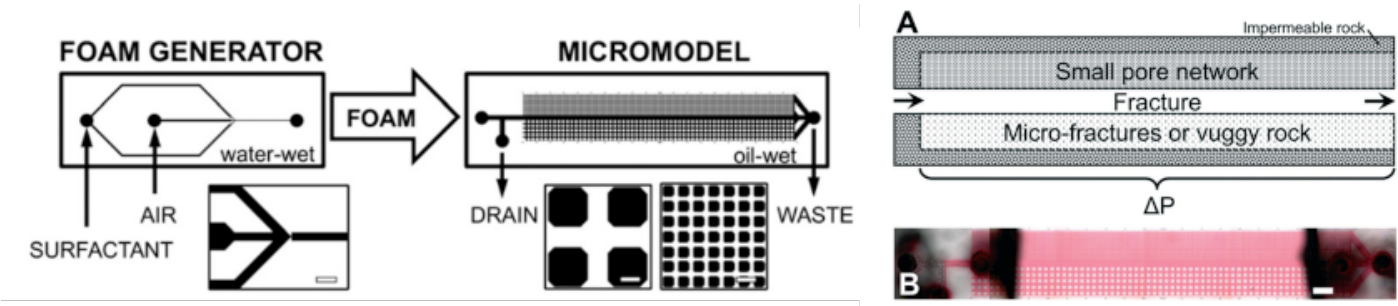

Figure 2.17: Flow-focusing device and micromodel showing permeability contrast [7]

Figure 2.18 compares oil displacement by water, water-alternating-gas (WAG), and foam injection in the micromodel. Foam improved sweep efficiency and oil displacement compared with other injection schemes. Bubble resistance in the fracture and high-permeability zones effectively displaced trapped oil in the low-permeability region, increasing local pressure gradients into the system enough to overcome the low-permeability capillary entry pressure. The entrapment of bubbles in foam resulted in a larger pressure drop and apparent viscosity.
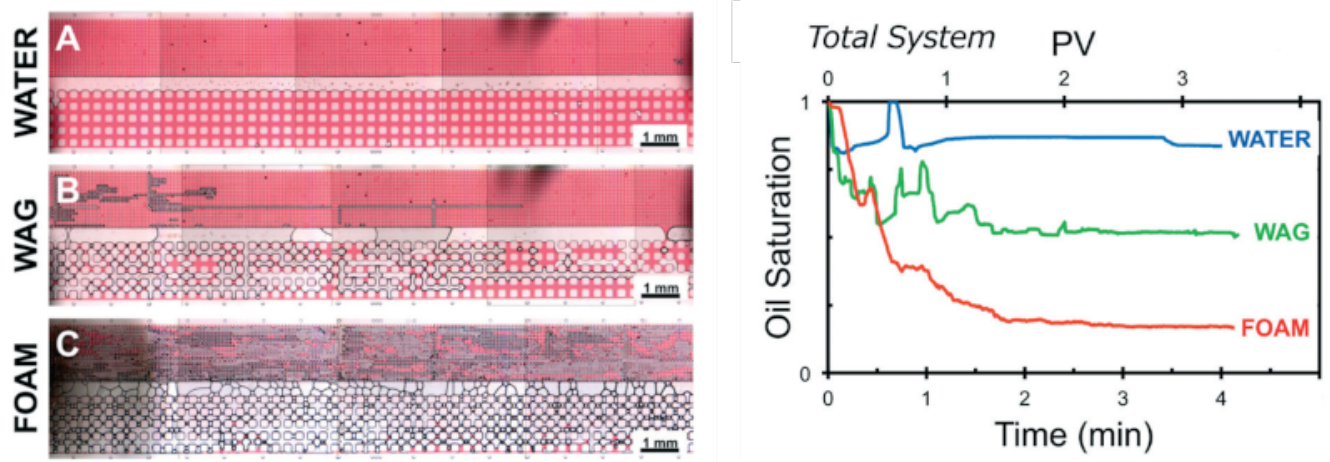

Figure 2.18: Foam sweep efficiency comparing with water and WAG [7] 
Surfactant-alternating-gas performance on oil displacement was studied by Salehi et al. in 2014 [44]. They used bead pack experiments to optimize the SAG ratio (ratio of volume of surfactant solution to volume of gas) and the surfactant concentration to obtain the maximum oil removal efficiency.

During the studies with SAG injection, the injection pressure was found to be enhanced due to foam formation inside the device. Figure 2.19 [44] compares oil recovery from waterflooding, gas flooding, and water-alternatinggas; oil recovery in SAG method is higher. The values are about $87 \%$ for SAG, $70 \%$ for WAG, $66 \%$ for waterflooding and $59 \%$ for gas flooding.

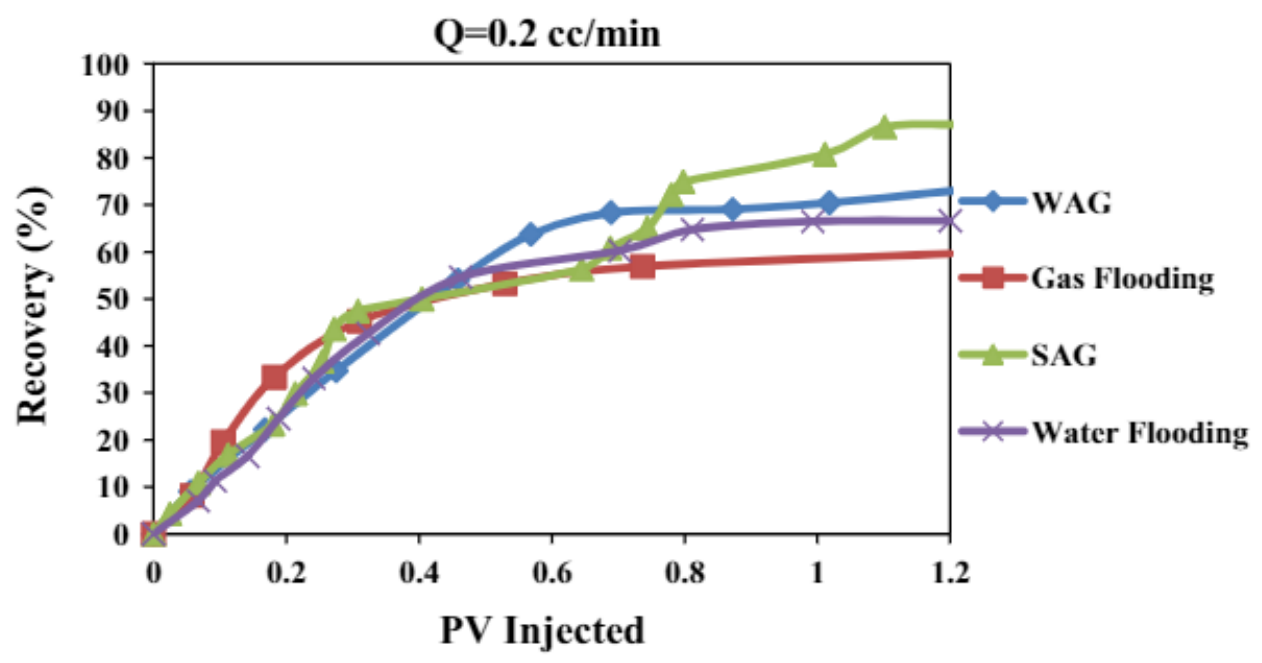

Figure 2.19: Recovery factors of SAG, WAG, water injection and gas injection $[44]$

Kamal et al. (2017) [45] evaluated surfactant type and concentration in core-flooding experiments during SAG injection. The idea was to study the effect on capillary trapping of gas in different types of rocks and surfactant concentrations. They measured pressure gradients during the whole experiment and residual gas saturation in the samples after injection. The results are summarized in Figure 2.20, which shows the effect of concentration. Increasing the concentration of a specific surfactant from $0.01 \%$ to $0.1 \%$ causes more than $200 \%$ increase in the pressure gradient across the sample. This increase indicates foam generation that leads to better gas trapping and mobility reduction. 


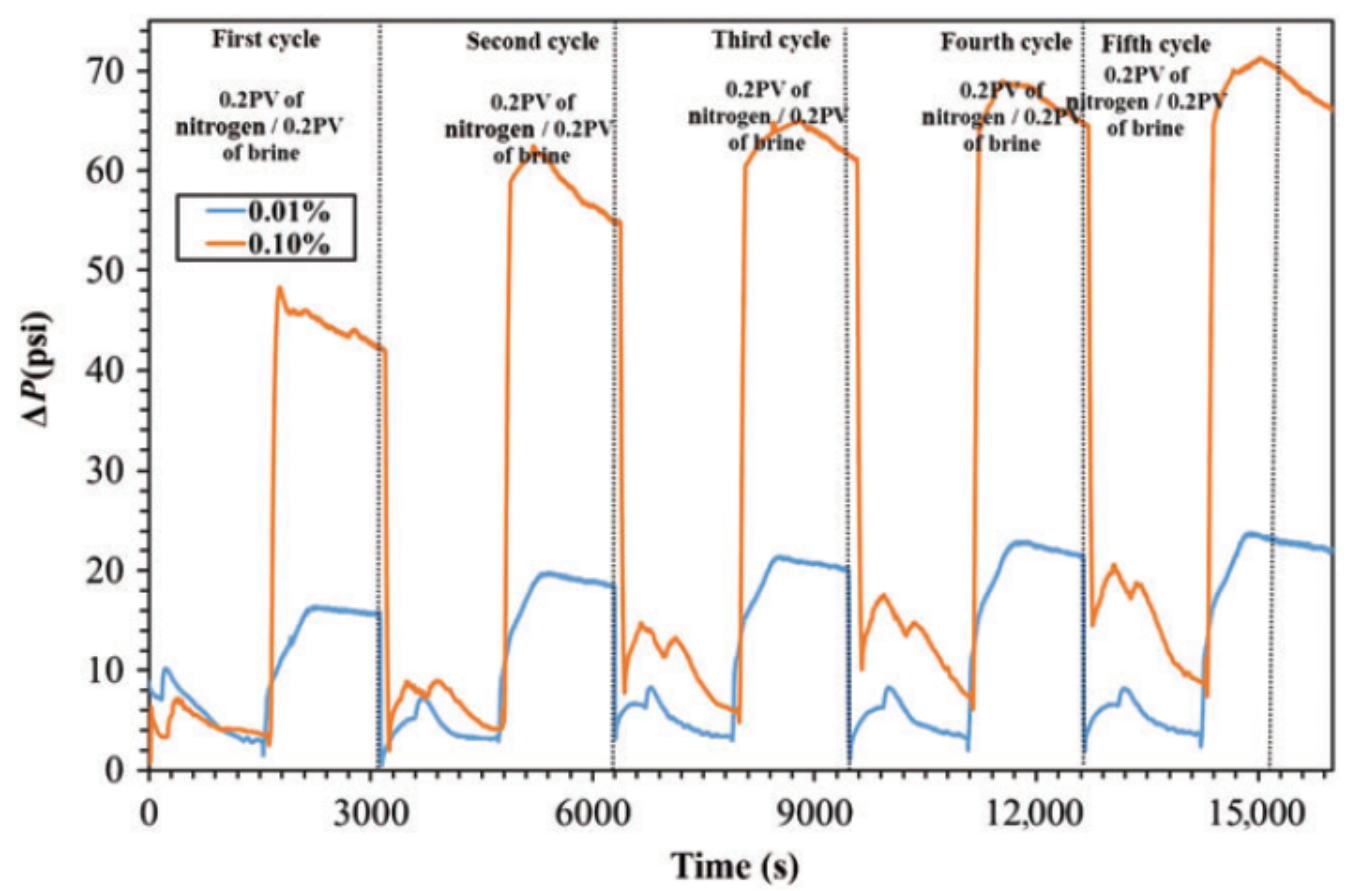

Figure 2.20: Pressure gradient across the sample at two different concentrations $[45]$

Wang et al. in 2020 [46] investigated through micro-scale visualization the multi-phase flow characteristics during water-alternating-gas injection in a glass micromodel. Figure 2.21 shows the device, that was fabricated to replicate a specific reservoir rock. Visual investigation provided valuable information on multi-phase flow peculiarities and displacement phenomena. A quantitative study was conducted on the volumetric sweep efficiency, oil displacement efficiency and residual oil distribution.

They concluded that the multiphase flow system is influenced by pore throat morphology, wettability, and injection parameters (injection rate and gas-water ratio). Also, that WAG injection combines the advantages of water and $\mathrm{CO}_{2}$ injection with a large sweep range and high oil displacement efficiency [46]. 

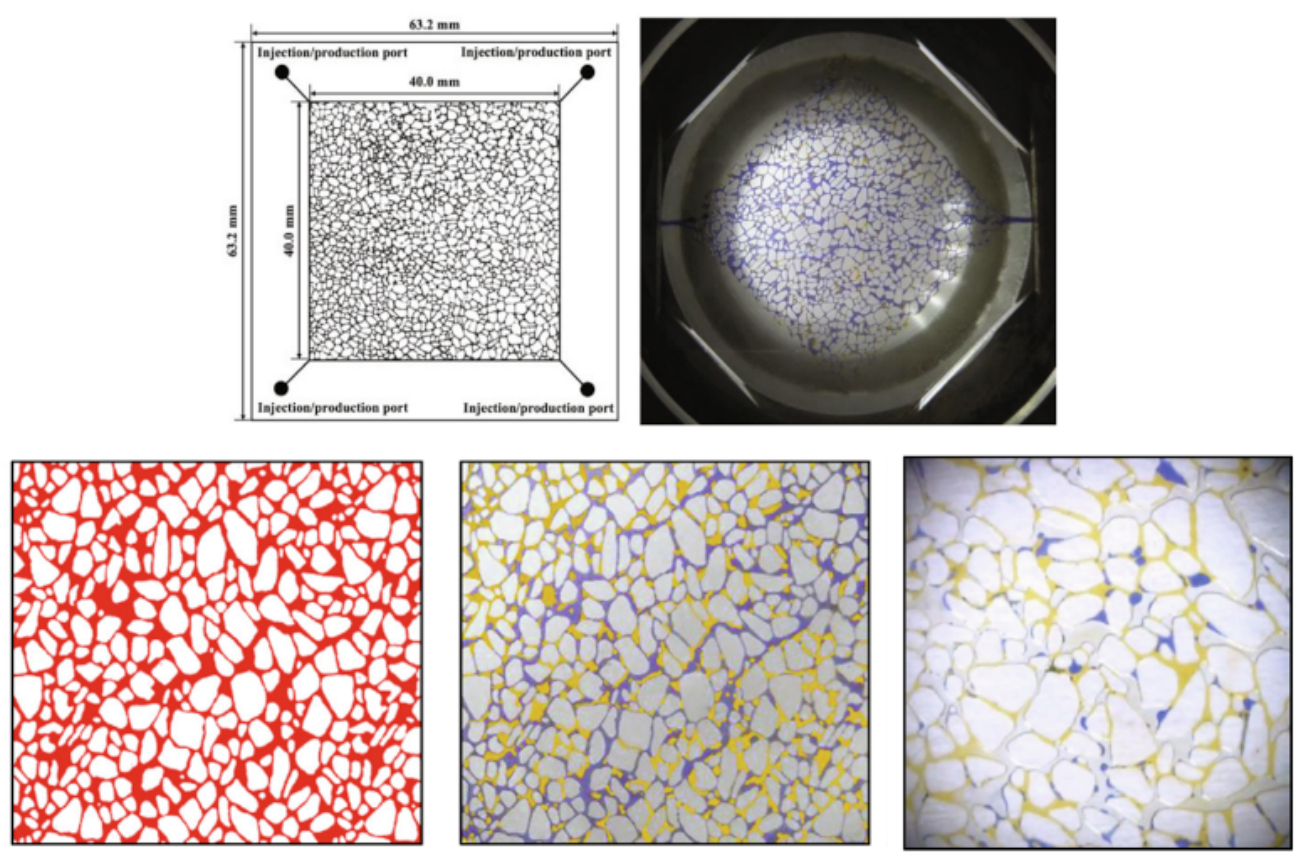

Figure 2.21: Porous media device used by Wang (oil was dyed in yellow, water in blue and gas remains transparent) [46]

In 2020, Li and Prigiobbe [47] conducted experiments in a glass porous medium device to better understand the impact of nanoparticles on foam generation processes and stability. A high-speed camera and a pressure transducer were used to monitor foam evolution in drainage and co-injection tests. They also used an algorithm based on a convolutional neural network (Figure 2.22) to determine the generation kinetics. The images obtained during the experiment show a foam generation process characterized by different mechanisms: early snap-off, followed by lamella-division, and finally leave-behind [47].
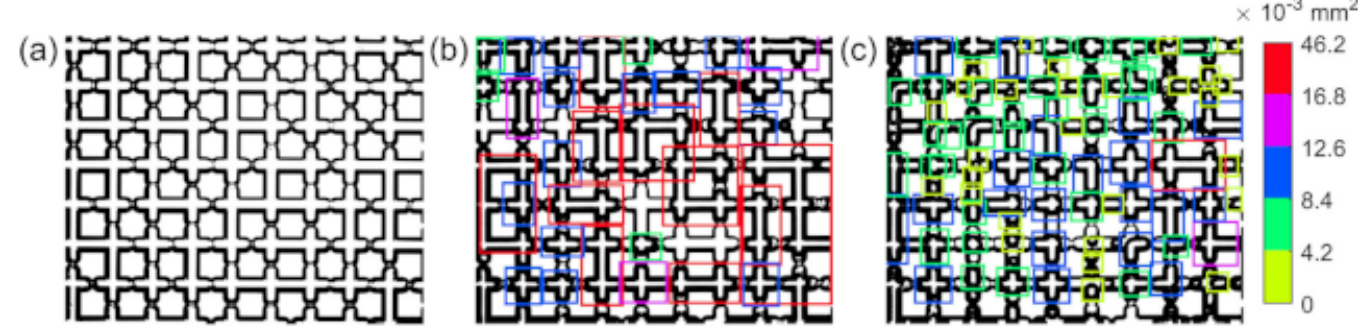

Figure 2.22: Bubble recognition a) Device saturated with surfactant solution without bubbles b) Micromodel with coarse foam c) Micromodel with fine foam [47]

Figure 2.23(a) reports the number of bubbles $(N)$ as a function of time for different gas rates [47]. The images were analyzed in early period of the foam generation when bubbles appeared for the first time in the observation area. 
$N$ increases following an almost constant slope until reaching a plateau. The pressure drop is also reported as a function of the pore volume injected (Figure 2.23(b)). It's possible to see that, in all cases, that pressure follows the same trend, initially increasing, reaching a maximum value and then decreasing, stabilizing around a constant value. Pressure evolution and the number of bubbles generated clearly depend on the gas rate [47].
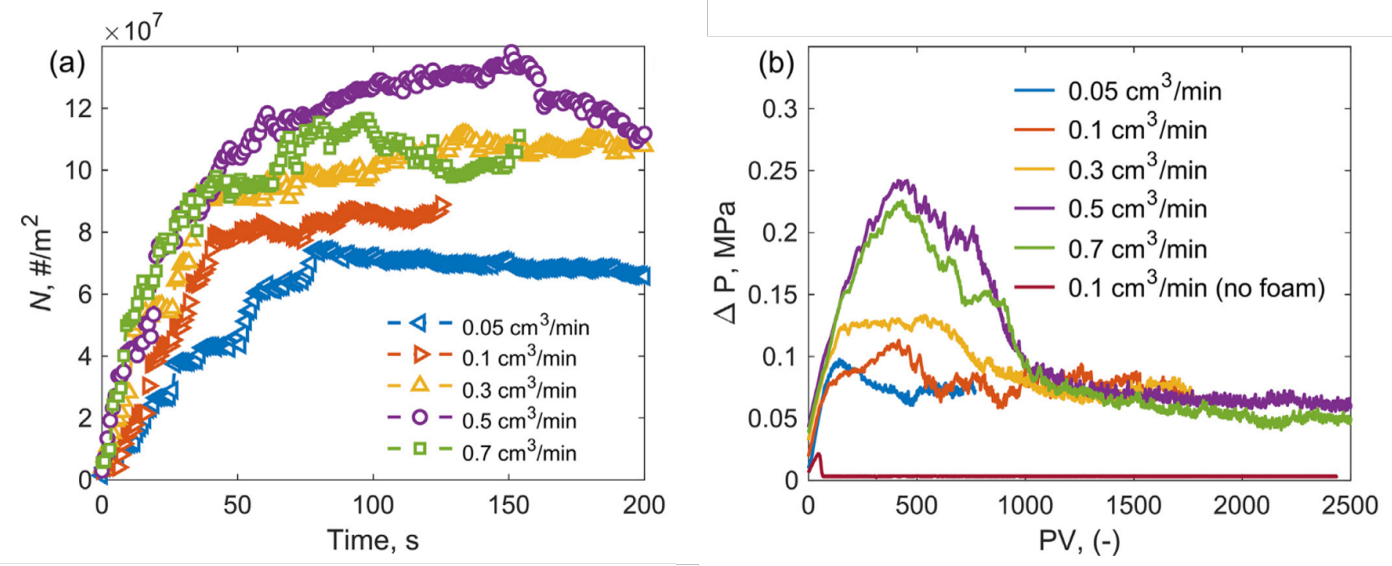

Figure 2.23: (a) Lamellae number as a function of time for different gas flow rates (b) Pressure $\mathrm{x}$ injected pore volume (PV) [47]

In 2021, Kai Li et al. [48] used a 1-meter-long transparent model fracture to study foam stability. Pre-generated foam was injected and its texture, apparent viscosity and fraction of trapped gas was analyzed using a highspeed camera. They divided the water-wet model plate in six sections (Figure 2.24) and obtained time- and location-averaged results. One interesting finding regarding foam texture in these sections is a result of in situ foam generation. Comparing the sections in Figure 2.25 it is noticed that bubble texture becomes finer along the fracture, and this is explained by lamella division, that was found to be the principal mechanism of foam generation inside the fracture. In the image (Figure 2.25) gas is shown in black and liquid in white. 


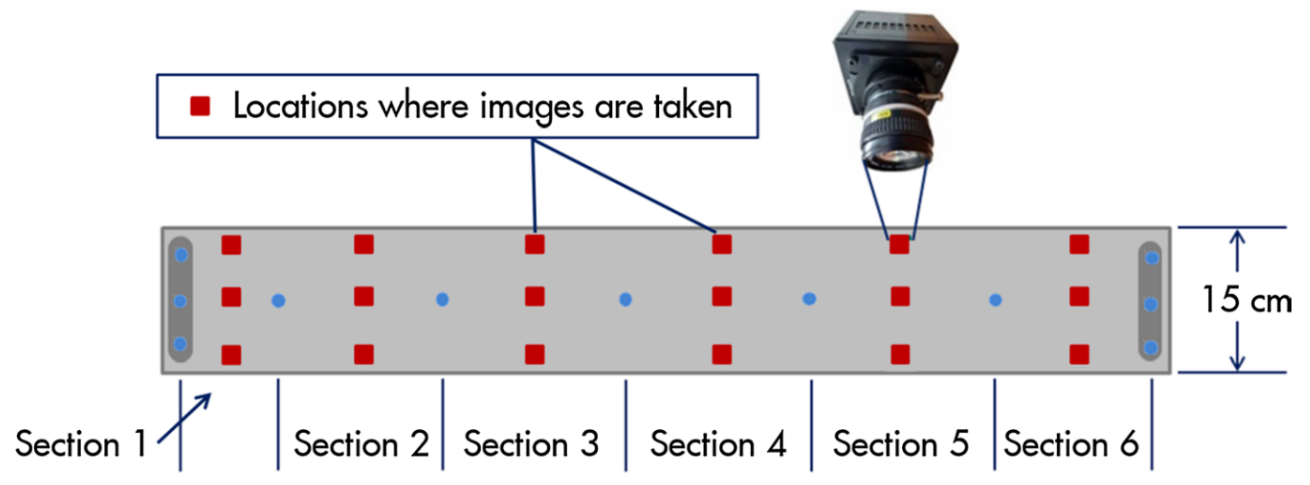

Figure 2.24: Setup of the model fracture showing the six sections [48]
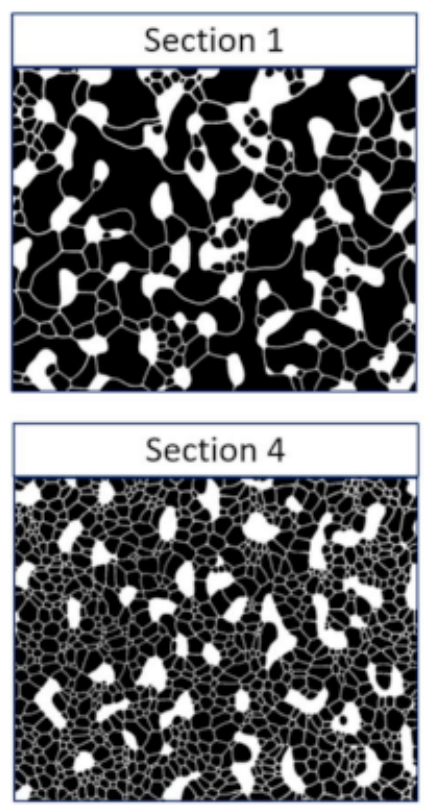
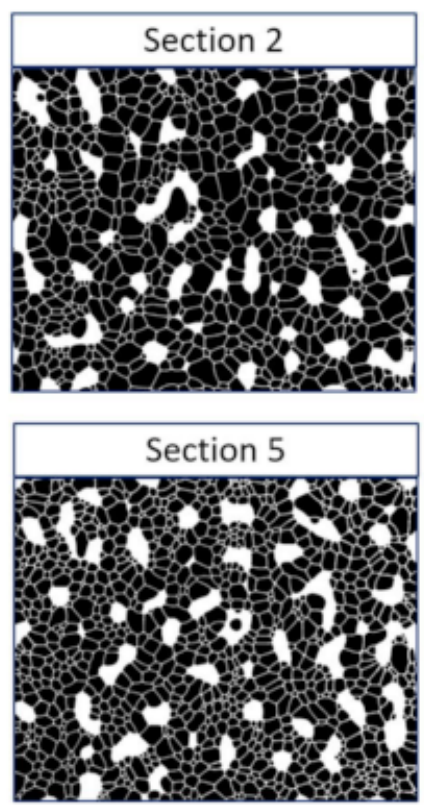
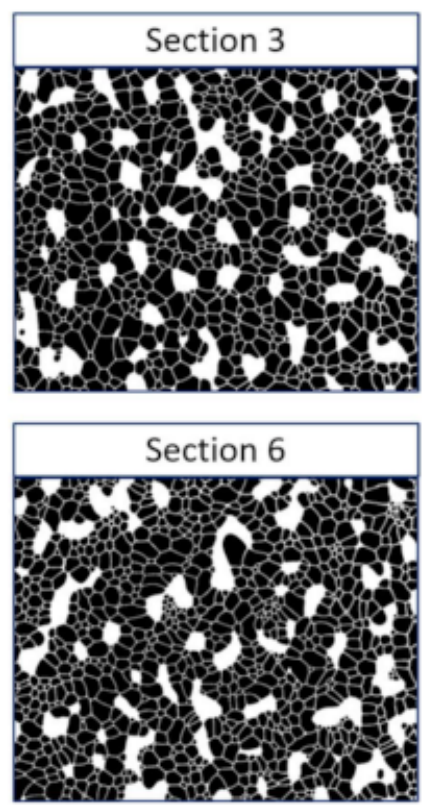

Figure 2.25: Evolution of bubble density and sizes at different sections along the fracture. [48]

Most studies on foam formation during surfactant-alternating-gas injection and the use of foams as an EOR method focus on the macroscopic relation between imposed flow rate $Q$ and $\Delta P$, without going into detail on pore scale phenomena.

Previous works studied surfactant concentration impact on foam behavior in core-flooding experiments [29] but not fully visualizing the dynamics. And the observation of foam evolution was carried out in another work [47] but not evaluating the surfactant concentration impact. 


\section{6}

\section{Objectives}

This work focuses on the impact of surfactant solution concentration on foam generation during its drainage by gas injection in porous media and on oil displacement by SAG injection through a $2 \mathrm{D}$ porous medium using a microfluidic system. Parameters such as pressure behavior during gas injection, lamellae formation, and phase distribution will be considered in this study.

The present study is divided in two parts. The first one studies the foam formation process in porous media when gas is injected at a constant volumetric flow rate in a surfactant solution saturated micromodel. The second part explores the importance of foam formation during SAG as an EOR method with the objective of increasing the volume of displaced oil.

The main goal is to visualize the pore scale phenomena and obtain macroscopic information from the pore-scale analysis that can be used to improve continuous model of foam formation and flow in porous media. 


\section{3}

\section{Materials and methods}

As previously discussed, two different pore scale analyses were developed using glass micromodels of porous media. The first one was focused on the understanding of foam formation during displacement of surfactant solution by gas injection. This study is presented in Chapter 4 . The second analysis focused on the study of SAG process, and is presented in Chapter 5. Fluids and glass device used in both analyses are described in this chapter.

\section{1}

\section{Materials}

\subsection{1}

\section{Surfactant}

Surfactants, also known as surface active agents, are amphiphilic molecules consisting of a hydrophilic part, referred to as the head, and a hydrophobic part, referred as the tail. The surfactant head can be anionic, cationic, nonionic or zwitterionic. The tail consists of linear or branched hydrocarbons chain. Aromatic groups and haloalkanes also may be present in the surfactant tails [49].

When dissolved in water, the amphiphilic feature of surfactants leads to the segregation of the hydrophobic tails from water and to the exposure of the hydrophilic heads, which results in the formation of aggregates above a threshold concentration, known as critical micelle concentration (CMC). Above the CMC the micelles coexist with single dispersed surfactant molecules, the monomers. And additional surfactants added to the system go to micelles [49].

In the experiments reported in this thesis, we used sodium dodecyl sulfate (SDS), also known as sodium lauryl sulfate (SLS). It is an anionic surfactant (Vetec Quimica Fina) with formula $\mathrm{C}_{12} \mathrm{H}_{25} \mathrm{NaSO}_{4}$ (Figure 3.1), molecular weight of $288.38 \mathrm{~g} / \mathrm{mol}$ and purity $90 \%$ in deionized water $(18.2 \mathrm{M} \Omega \mathrm{cm})$. The solution was prepared by dissolving the powder surfactant in deionized water, filtrated through a $0.45 \mu \mathrm{m}$ filter. Aqueous dye was added to the surfactant solution to better distinguish liquid from other fluids and glass matrix in the visualization experiments. 


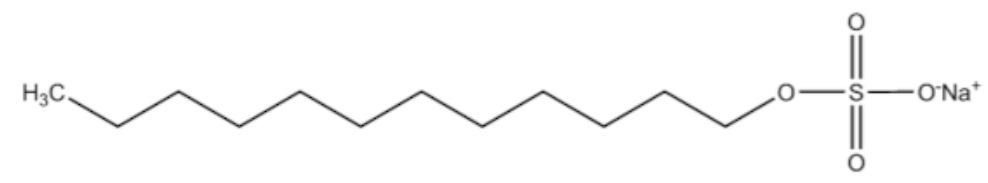

Figure 3.1: Sodium dodecyl sulfate (SDS)

Surface tension measurements were carried out in aqueous solutions of SDS in order to determine the CMC of the system. All measurements were done on a DCAT25 tensiometer by DataPhysics Instruments (Figure 3.2) using a Wilhelmy plate. The reported values of the surface tension were obtained at constant temperature of $23^{\circ} \mathrm{C}$. The equilibrium surface tension of the water with red dye used for the preparation of solutions was $59.7 \mathrm{mN} / \mathrm{m}$.

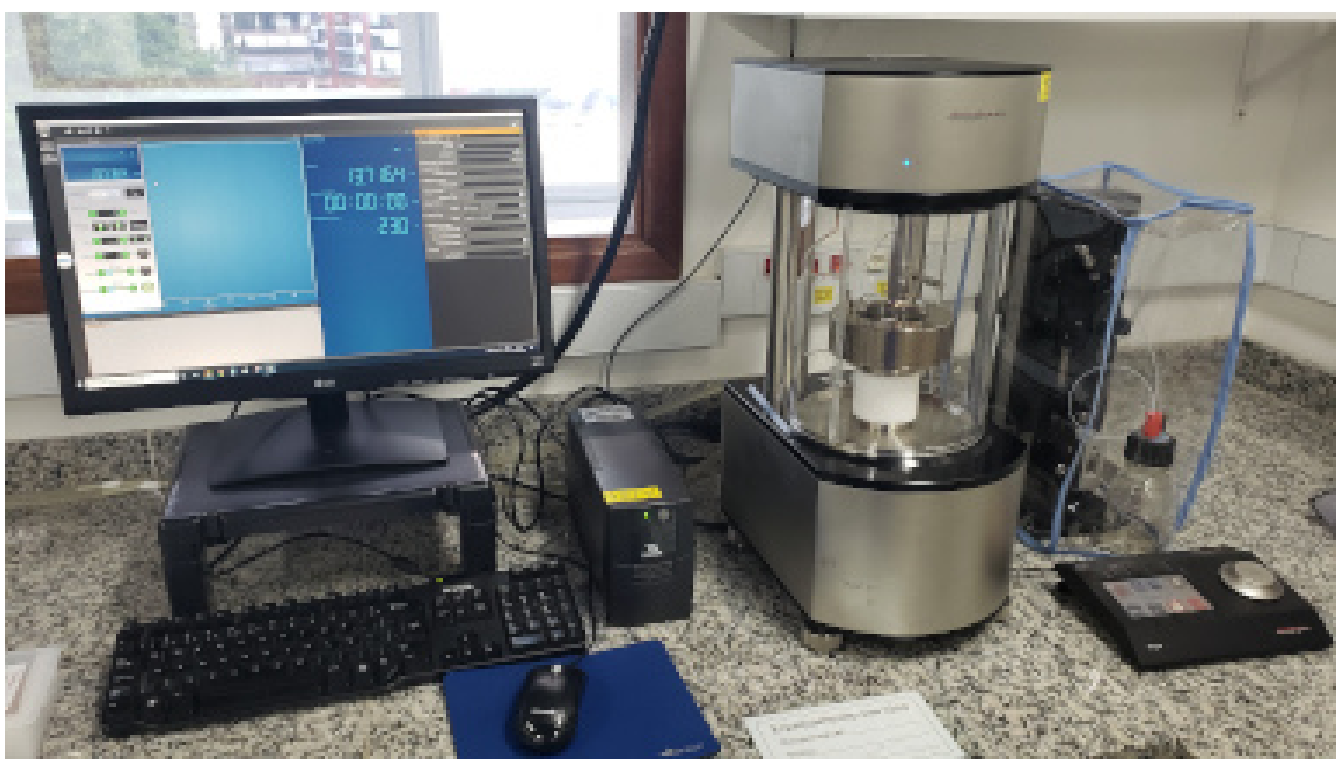

Figure 3.2: DataPhysics Tensiometer

Surfactant molecules exist in three configurations in a surfactant solution above the CMC: they are dispersed as monomers, as aggregates (micelles), or adsorbed as a film along the air/water interface. Individual surfactant molecules are constantly exchanged between the bulk and the micelles, so the micelles are in a dynamic equilibrium with them. In addition, the micelles are constantly disintegrating and regenerating [50].

Many factors influence the value of $\mathrm{CMC}$, including temperature, $\mathrm{pH}$, and the presence of organic modifiers. Taking the surface tension of SDS solutions as a function of concentration, the behavior expected was a sharp break at the $\mathrm{CMC}$ value, the surface tension decreases with increasing concentration until $\mathrm{CMC}$ is reached, after that it remains constant, as sketched in Figure 3.3. 


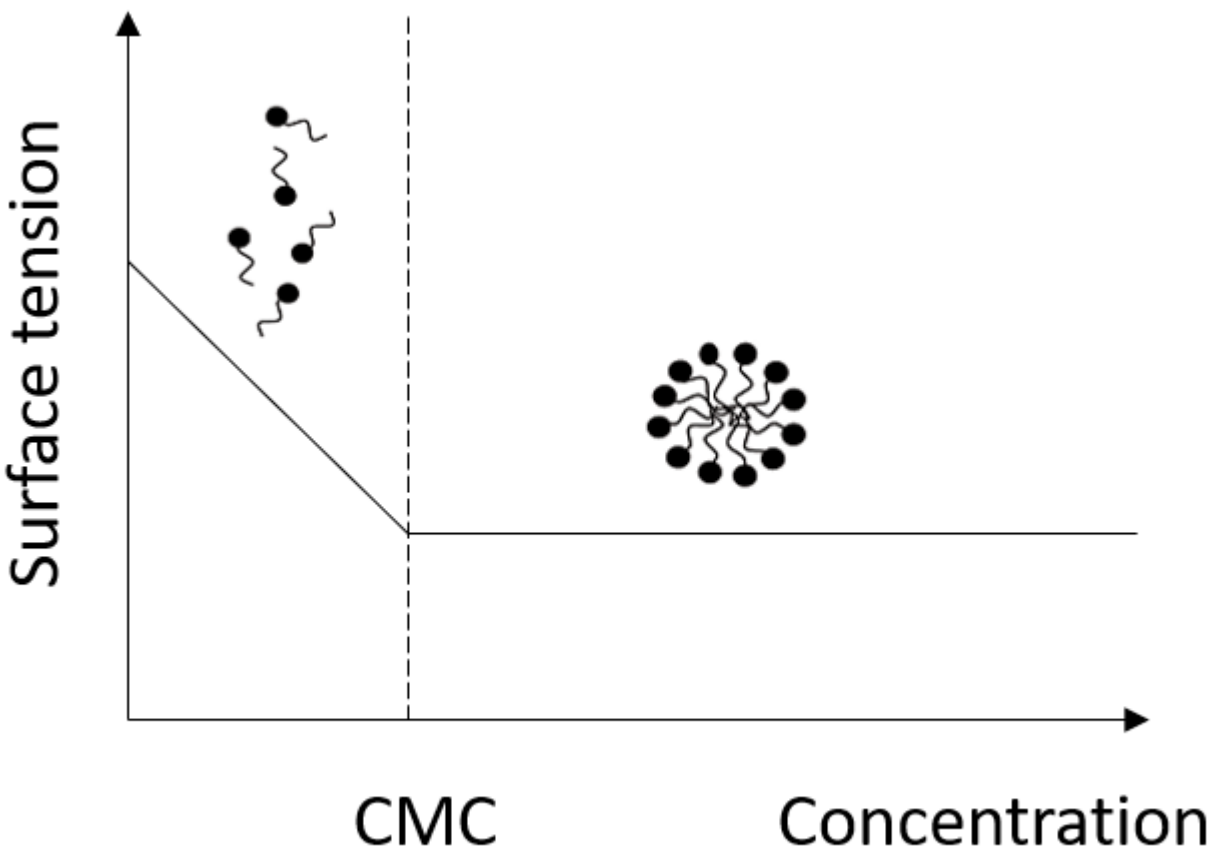

Figure 3.3: Surface tension as a function of concentration

The measured equilibrium surface tension as a function of the surfactant concentration is presented in Figure 3.4. The interfacial tension value stabilizes at $34.4 \mathrm{mN} / \mathrm{m}$ at high enough surfactant concentration. Figure 3.5 presents a zoom of the data for concentration bellow $1 \mathrm{~g} / \mathrm{L}$. The surface tension drops dramatically as concentration rises until reaching a concentration close to 0.5 $\mathrm{g} / \mathrm{L}$, at which point it begins to rise, resulting in a visible minimum in the surface tension curve.

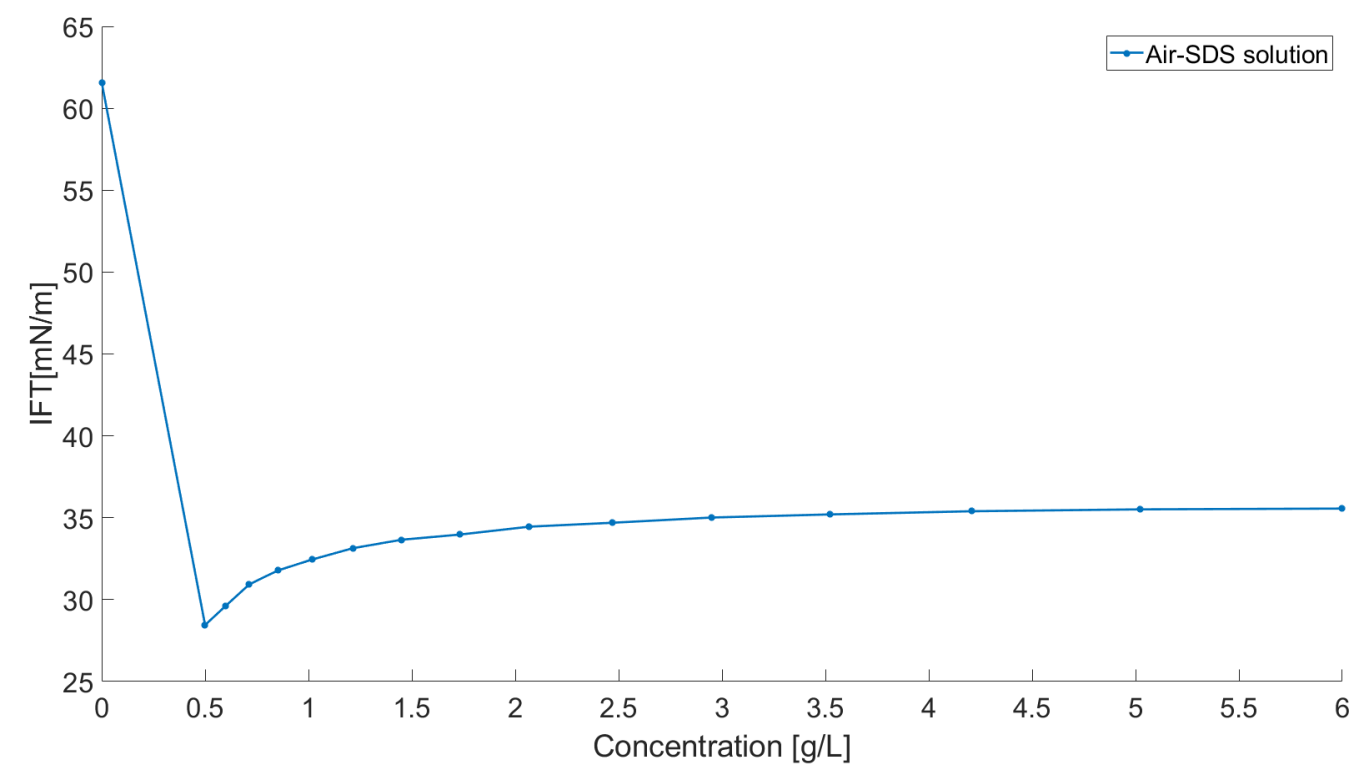

Figure 3.4: Air-SDS solution interfacial tension 


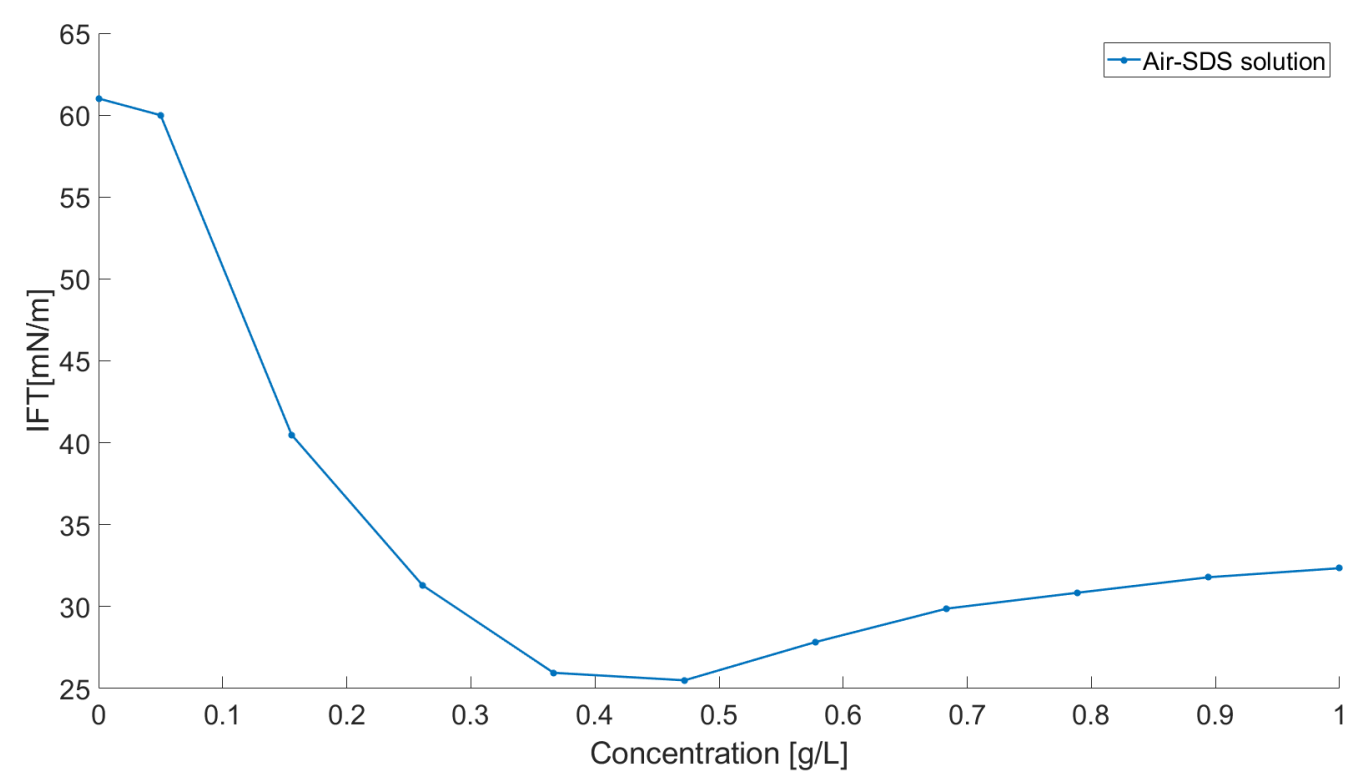

Figure 3.5: Air-SDS interfacial tension near CMC

This minimum in the surface tension value was also reported in other studies [51, 52], and is explained by the presence of impurities that absorb at the air-liquid interface and have a surface activity greater than the surfactant itself. The impurities might be dodecyl alcohol or inorganic salts as SDS is not $100 \%$ pure.

When the system is impure, the determination of the CMC becomes more complicated. As the transition between monomers and micelles is gradual, the term "critical micelle concentration" isn't appropriate in this circumstance. At this time, the term "micellar dissociation concentration" (MDC) makes more sense [53].

Analyzing the curve in Figure 3.4 from right to left, the surfactant concentration at which the mixed micelles seem to be dissociating is when the curve starts to fall. The value to be considered here is between 2 and 3 $\mathrm{g} / \mathrm{L}$. According to the literature, the expected concentration value is $8.2 \mathrm{mM}$, equal to $2.36 \mathrm{~g} / \mathrm{L}$, this is the reference $\mathrm{CMC} / \mathrm{MDC}$ value used in the analysis of the results.

When air flows through a surfactant solution, it creates a large amount of additional interfacial area. An adsorbed film of surfactant molecules must be used to stabilize the increased interfacial area. These molecules are derived from the bulk solution, that contains monomers and micelles. Micelles need to break up as monomers to diffuse towards the recently formed surface. It is also important to consider that the curvature of the interfaces in the porous medium can affect the value of the interfacial tension.

Viscosity is another important parameter to be considered when studying 
surfactant solution displacement by gas at the pore scale. The dynamic viscosity of the surfactant solutions used in the experiments is presented in Table 3.1. It is possible to notice that the viscosity slightly increases as the surfactant concentration increases.

\begin{tabular}{c|c}
$\begin{array}{c}\text { Surfactant (SDS) } \\
\text { concentration (g/L) }\end{array}$ & Dynamic viscosity (cP) \\
\hline 0 & 0.939 \\
\hline 0.235 & 0.925 \\
\hline 0.47 & 0.925 \\
\hline 1.55 & 0.930 \\
\hline 3.1 & 0.939 \\
\hline 6.2 & 0.958 \\
\hline 15.5 & 1.024
\end{tabular}

Table 3.1: Dynamic viscosity for different surfactant concentrations

\subsection{2}

Air

The gas phase used in all experiments was air at room temperature. A $0.45 \mu \mathrm{m}$ PTFE inline filter was used to fill the gas syringe with air.

\subsection{3}

Oil

DRAKEOL®7 by Calumet Lubricants is a mixture of paraffinic and naphthenic hydrocarbons. It is a white mineral oil and was dyed with black color using an oil-based dye to allow visualization and differentiation of phases during the visualization experiments. The colors obtained with dye are shown in Figure 3.6. 


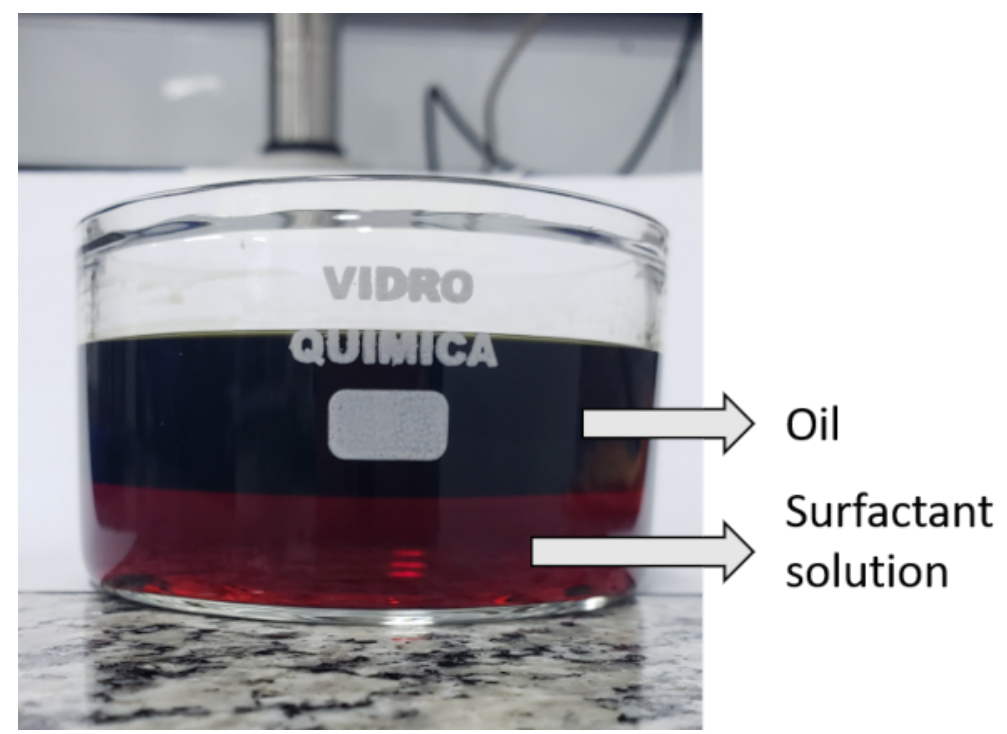

Figure 3.6: Oil and surfactant solution used for interfacial tension measurement

Figure 3.7 shows the curve of interfacial tension $(\sigma)$ between oil and surfactant solution at $23^{\circ} \mathrm{C}$ as a function of time. The concentration of the surfactant solution used in this measurement was $6.2 \mathrm{~g} / \mathrm{L}$, which is above the CMC. Equilibrium was reached after 30 minutes with a value of interfacial tension close to $4 \mathrm{mN} / \mathrm{m}$.

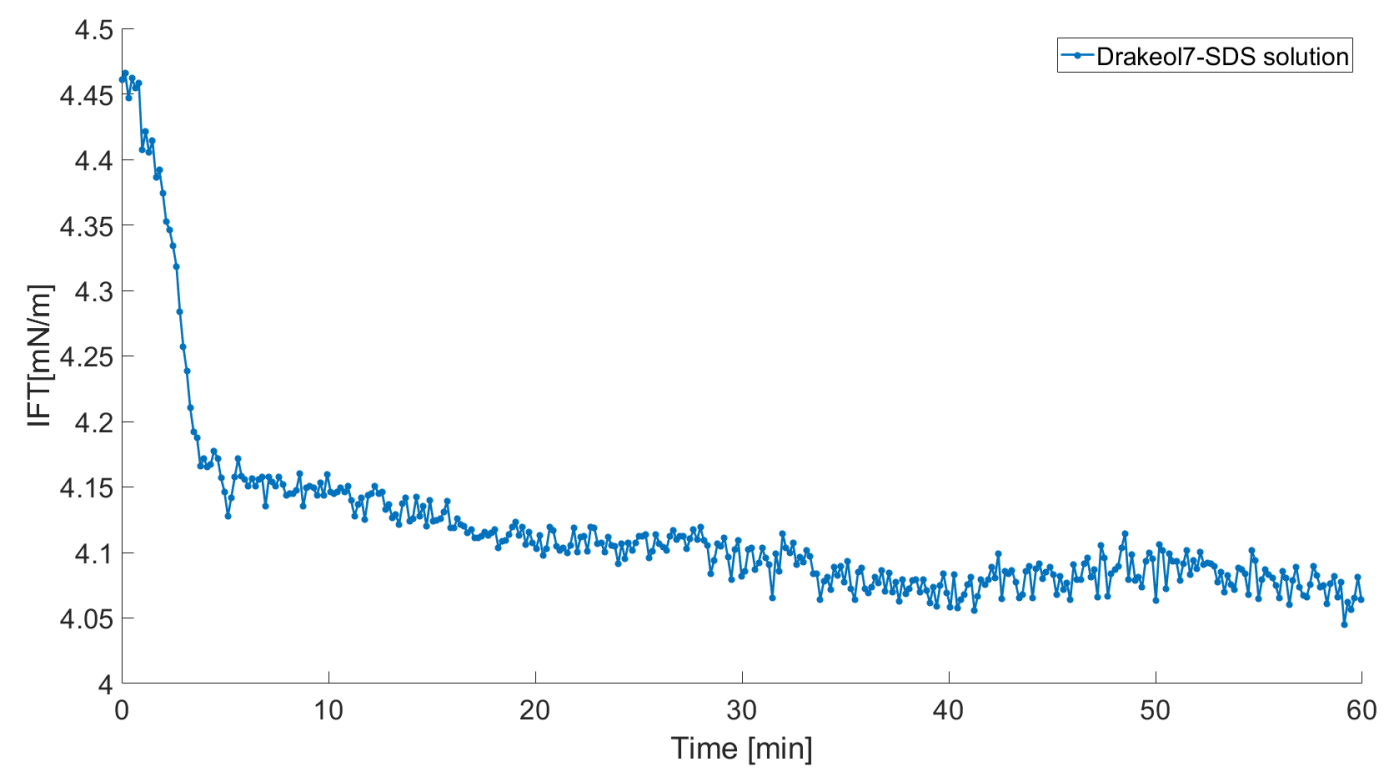

Figure 3.7: Oil-surfactant interfacial tension

Important physical properties such as density and viscosity were also measured. The oil density was $0.85 \mathrm{~g} / \mathrm{mL}$ and the dynamic viscosity obtained using a rotational rheometer DHR-3 from TA Instruments was $16.5 \mathrm{cP}$. 


\section{2}

\section{Porous Media Micromodel}

The flow through reservoir formation cannot be easily visualized. In order to visualize and study pore scale phenomena, transparent microfluidic devices representing a porous medium can be used.

The idea is to use microfluidics, a technology that process or manipulate very small volumes of fluid using channels with length scales of less than 100 $\mu \mathrm{m}$. The advantages are the reduced amount of fluids needed, the extensive range of applications and the optical access to analyze the fluid flow using optical techniques as cameras and microscopes.

In 2011, for the first time, a microfluidic device called "Reservoir-on-achip" was used to perform traditional waterflooding analysis [54]. The flow visualization provided specific information about the fluid distribution inside the porous medium. After that, many studies using silicon or glass-based micromodels were performed to understand multi-phase flow and fluid-solid interactions in EOR methods ([55, 7, 46, 56, 48]).

Fluids like crude oil, surfactant and polymer solutions, and gases like air, carbon dioxide and nitrogen are introduced into these transparent micromodels using specific pumps. The resulting flow patterns and behavior inside these devices are studied and analyzed.

The fluid injection experiments were performed on a microfluidic model porous medium, made with borosilicate glass and produced by Micronit (Figure 3.8). The micromodel is water-wet and has a porous matrix $20 \mathrm{~mm}$ long by $10 \mathrm{~mm}$ wide and $20 \mu \mathrm{m}$ etching-depth. Prior to the porous matrix, inlet and outlet channels act as flow distribution fractures that are $500 \mu \mathrm{m}$ wide. The pore volume is $2.3 \mu \mathrm{L}$ and matrix porosity is $57 \%$. The permeability of the model, according to the supplier, is $2.5 \mathrm{D}$. 

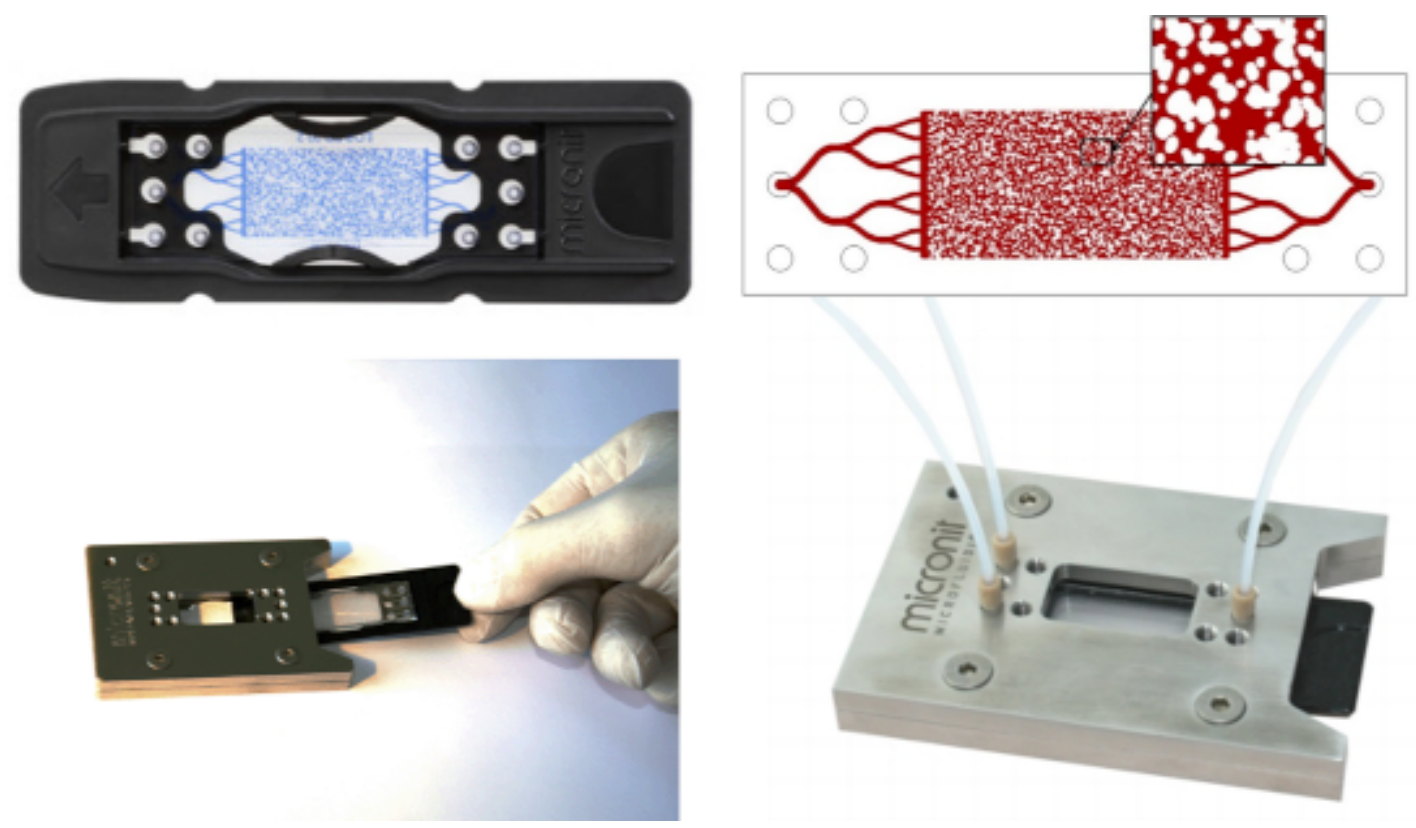

Figure 3.8: Micronit microfluidic device

The device was designed by randomly placing rock shape structures to resemble as much as possible the actual shape that is obtained by cutting a rock and scanning it. Throats and channels appear between the solid matrix structures as a result of this random design. This approach of random placement provides no information about throat size distribution. The approximate size of the smallest throat is $12 \mu \mathrm{m}$, while the largest throat is approximately $250 \mu \mathrm{m}$. The micromodel is a $2 \mathrm{D}$ device, so it is important to consider that the height $(20 \mu \mathrm{m})$ is the smallest dimension in the majority of pore throats. 


\section{4}

\section{Foam formation during drainage of a surfactant solution}

The analysis presented in this chapter was focused on foam formation during the displacement of a surfactant solution by a gas phase. Two parameters were evaluated: the surfactant concentration and the injection rate.

\section{1}

\section{Experimental setup and procedures}

The analysis is focused on a two-phase flow, with a gas phase and an aqueous phase, with or without surfactant. The fluid injection system (Figure 4.1) consisted of a syringe pump (Harvard Apparatus) used for injecting both phases. Fluid injection was performed using gas-tight glass syringes (Hamilton), the termination in Teflon with Luer-Lock coupling provides an easy connection with the $1 / 32$ " internal diameter tubing. A three-way valve was used to connect the pressure transducer.

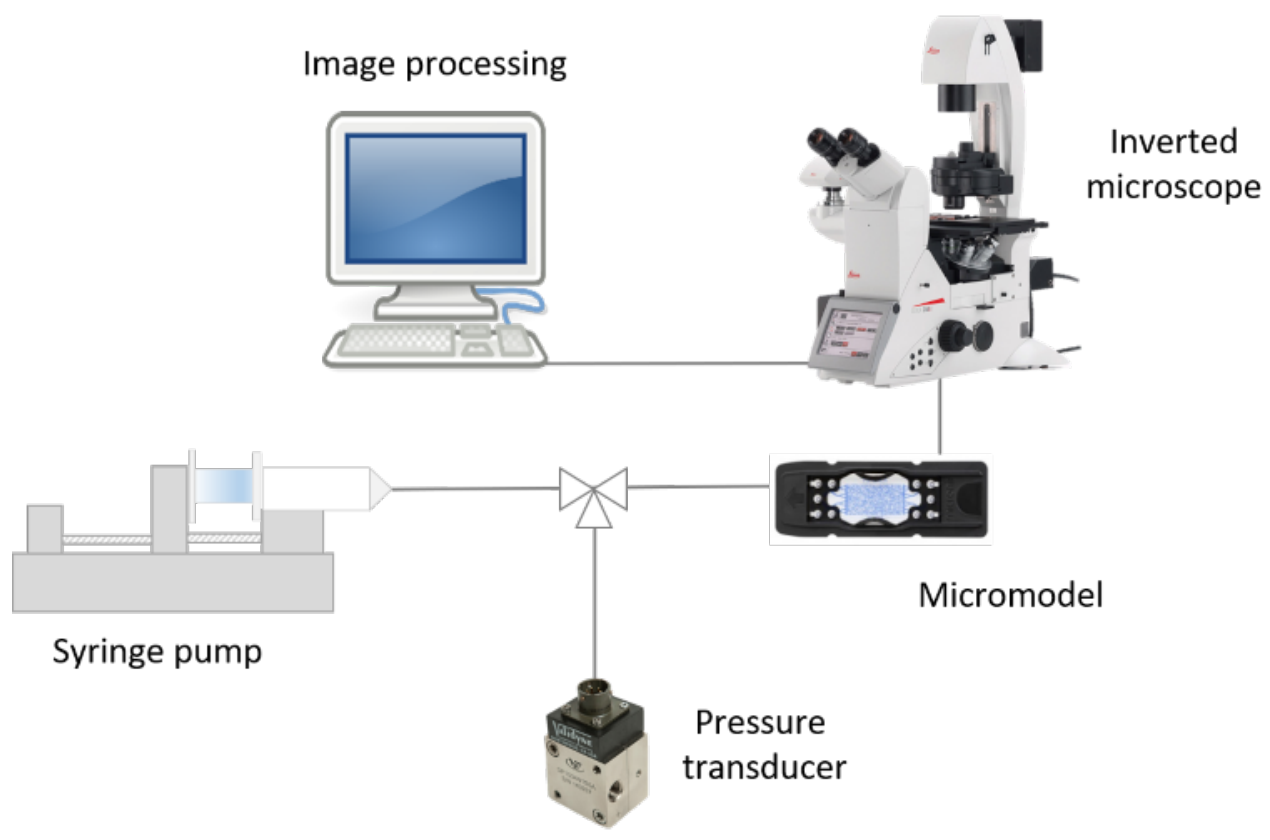

Figure 4.1: Scheme of the experimental setup

The differential pressure was acquired using a DP15TL pressure transducer produced by Validyne Engineering placed between the syringe pump 
and the microfluidic device (Figure 4.2). The pressure transducer employs interchangeable stainless-steel diaphragms well suited for this application. The diaphragms used have 0.5\% accuracy and pressure ranges of 0-5 psi and 0-20 psi. The outlet was open to atmosphere and inlet pressure was measured during the test. In the plots, the pressure is presented as a normalized pressure, defined as:

$$
\bar{P}(t)=\frac{P(t)-P_{\text {in }}}{P_{\text {in }}}
$$

in which $P_{i n}$ is the inlet pressure value when the gas enters the micromodel.

The microfluidic device was placed on the stage of an inverted microscope (Leica DMi8) for visualization. Leica MC170 HD camera was used to record the evolution of the phase distribution during the experiment, as shown in (Figure 4.2).
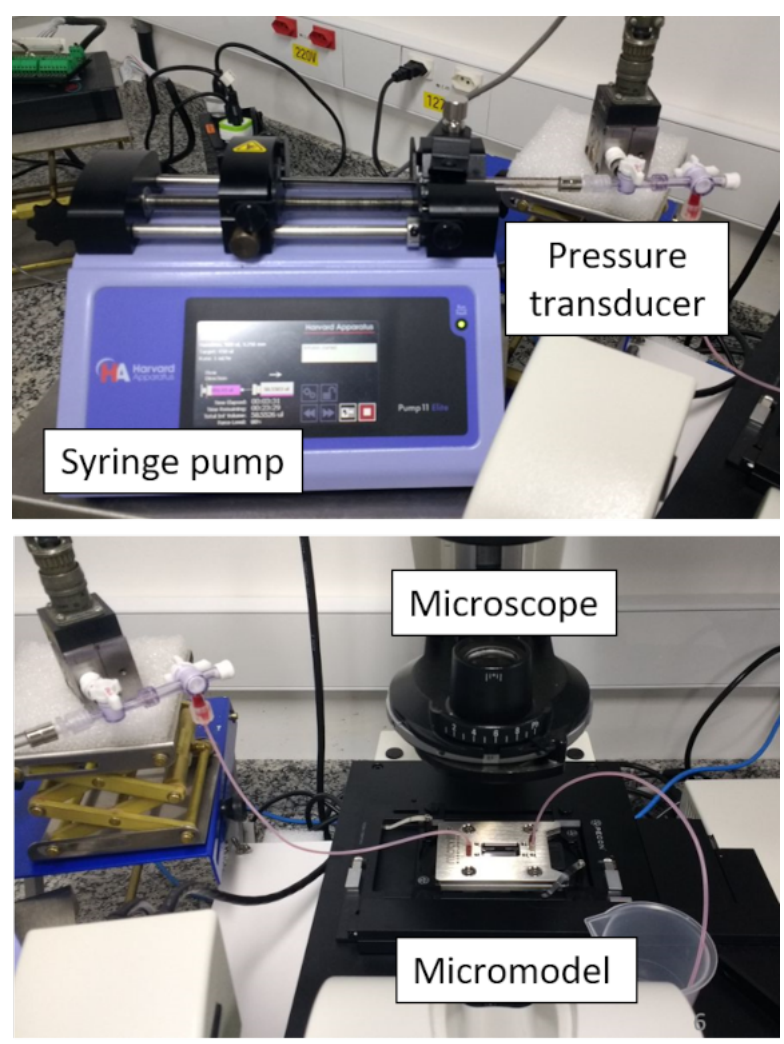

Figure 4.2: Experimental setup

In the experiments, the porous medium micromodel was initially saturated with the surfactant solution (SDS), then, gas-phase injection continued until the pressure reaches a steady-state condition. Surfactant concentration varied from $0 \mathrm{~g} / \mathrm{L}$ to $15.5 \mathrm{~g} / \mathrm{L}$, the maximum concentration is approximately $54 \mathrm{mM}, 6.5 \times \mathrm{CMC}$. 
The micromodel was first saturated with pure carbon dioxide prior to surfactant solution injection to ensure a complete saturation of the liquid phase. The procedure used to saturate the micromodel with the surfactant solution starts with injection at a very low flow rate $(0.1 \mathrm{ml} / \mathrm{h})$ overnight (approximately 10 hours), then the injection rate is increased to $0.5 \mathrm{ml} / \mathrm{h}$ for 2 hours and finally 1 hour at a very high injection rate, equivalent to $5 \mathrm{ml} / \mathrm{h}$. In total, more than 3,000 pore volumes are injected. Air was injected at a constant volumetric flow rate, equal to $1 \mathrm{ml} / \mathrm{h}$ or $2 \mathrm{ml} / \mathrm{h}$.

During gas injection and liquid displacement, multiple liquid films were formed from all mechanisms cited previously. The recorded images of the phase distribution were processed in order to determine the characteristics of the gas and liquid flow, to assess the evolution of foam texture, and measure the number of lamellae in the pore space.

The gas injection is stopped when it is noticed that the steady state has been reached.

\section{2}

\section{Image Processing}

The image processing tool used to process the recorded images was Fiji (Fiji Is Just ImageJ) that has built-in plugins that facilitates scientific image analysis. It is an open source package for a wide range of applications. The images used in the analysis were taken from a specific area of the microdevice (yellow rectangle), as shown in the Figure 4.3, for different time steps during the foam formation experiment.

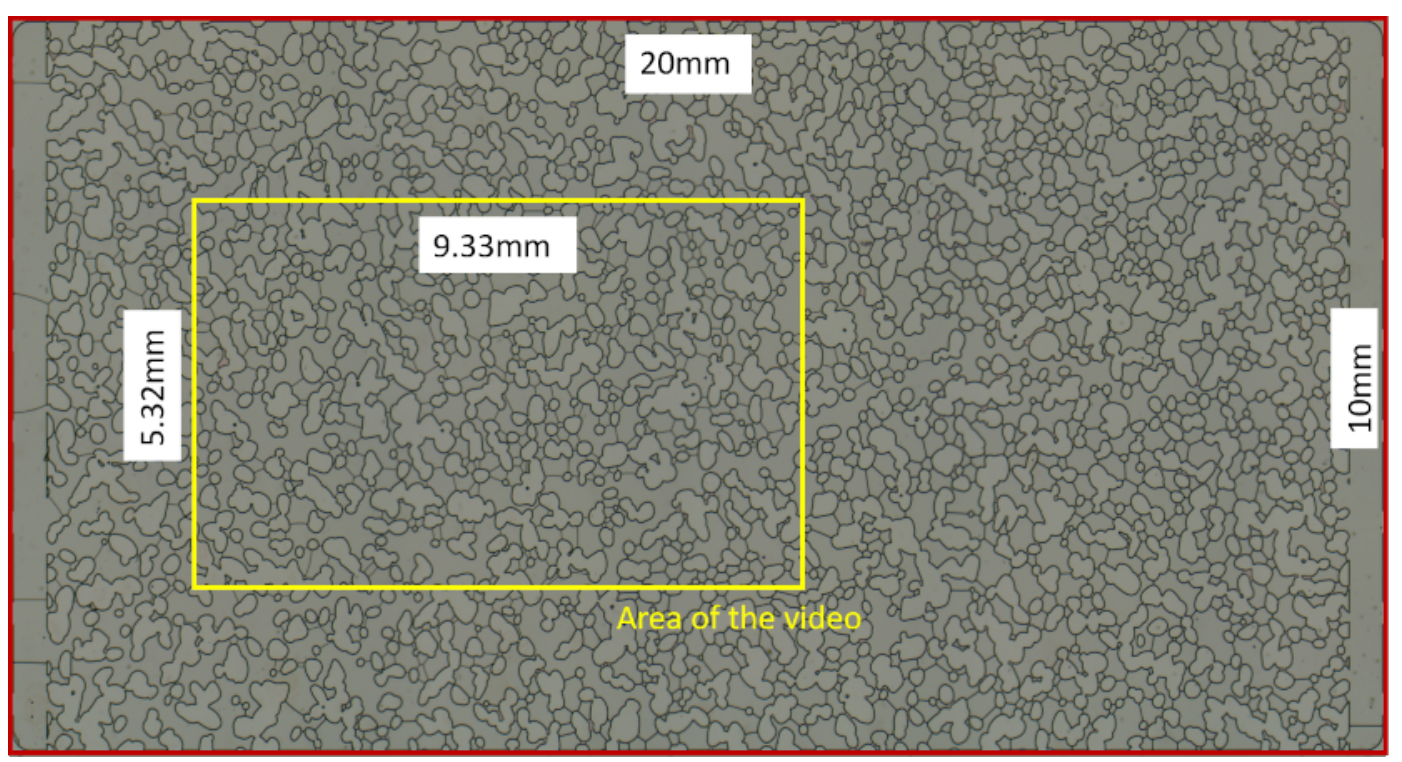

Figure 4.3: The area analyzed in the micromodel 
The procedure used to determine the number of lamellae is discussed next. First, an image with the device completely saturated with air was used to define the configuration of the solid matrix and pore space, named as Mask (Figure 4.4).

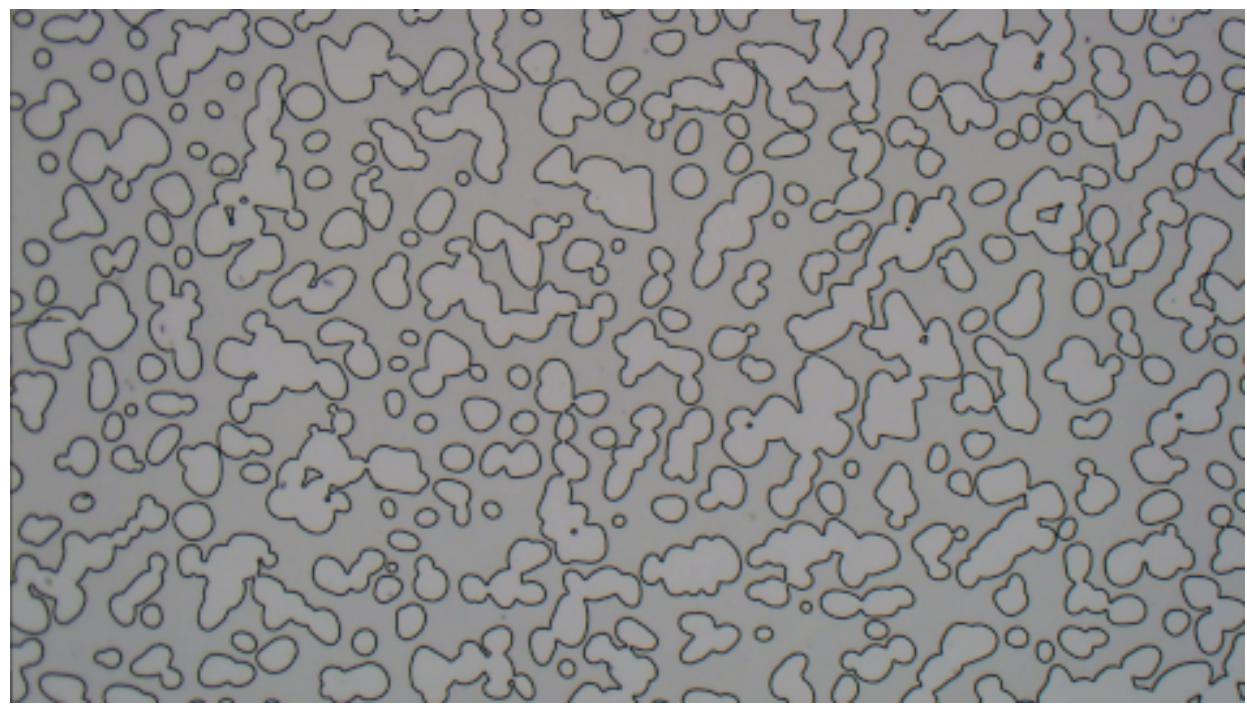

Figure 4.4: Device filled with air

During the surfactant solution displacement by gas injection, a frame obtained every 10 seconds of the video recorded was analyzed. Figure 4.5 presents an example of such image, named as Lamellae image. The presence of liquid films defining multiple gas bubbles is clear.

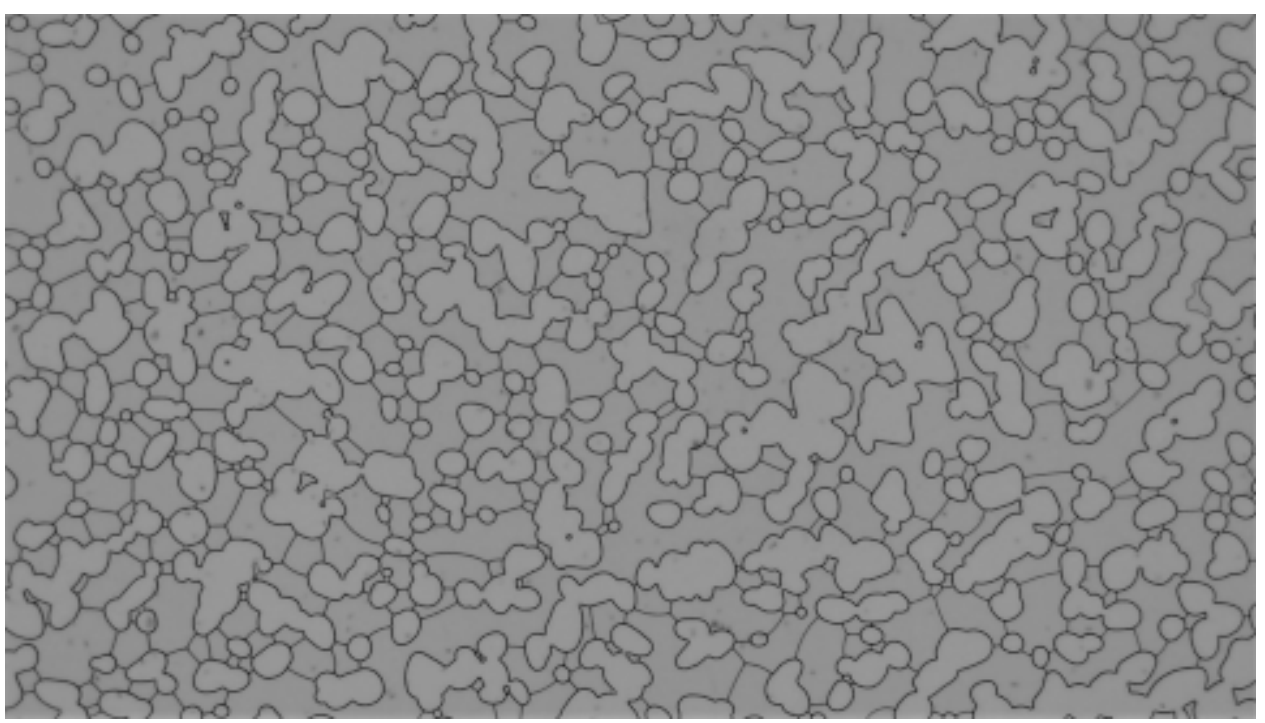

Figure 4.5: Device filled with liquid films (lamellae)

Figure 4.6 presents a flowchart with the steps involved in the image analysis process. The inputs are the images shown in Figures 4.4 and 4.5. 


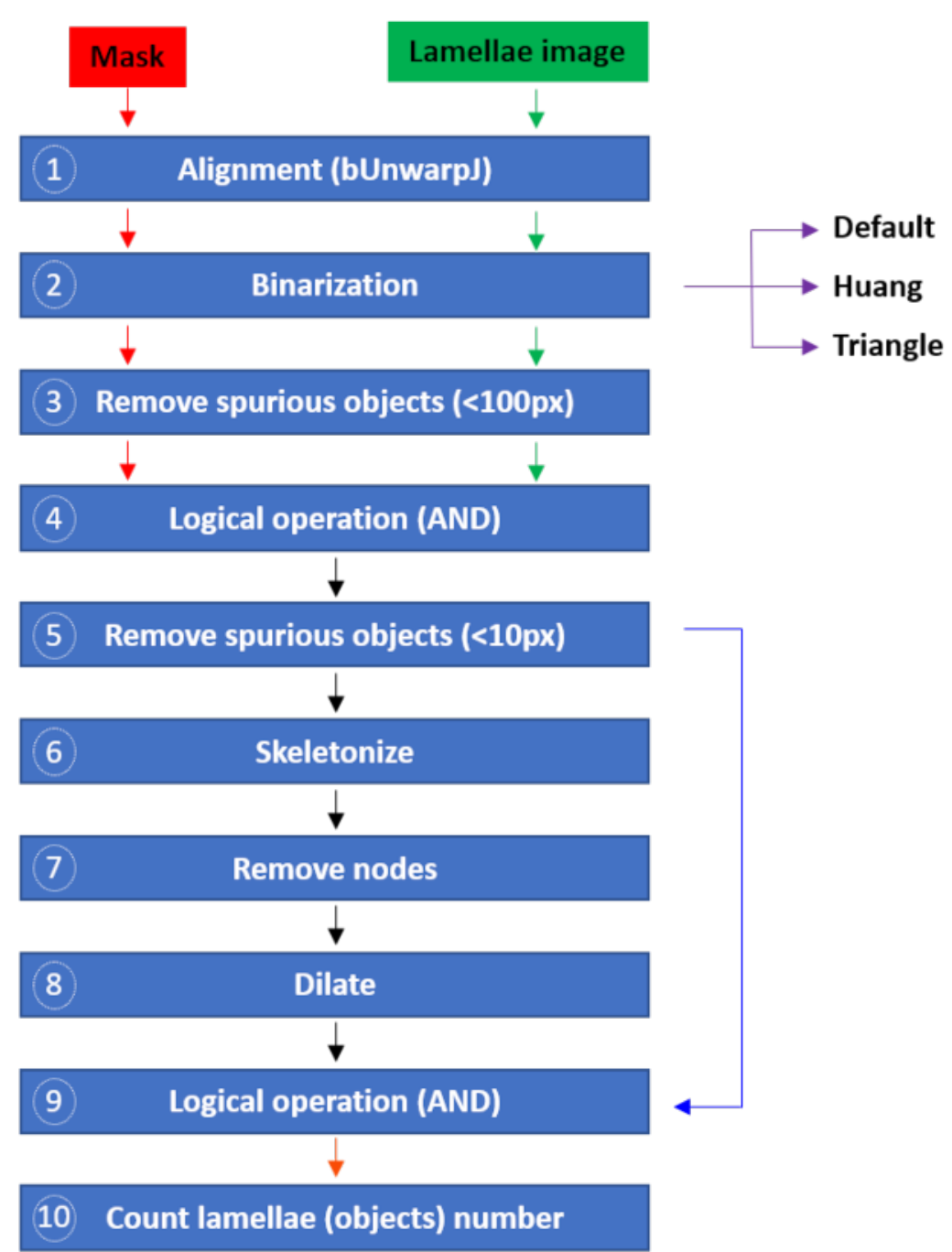

Figure 4.6: Image processing flowchart

In the first operation (Figure 4.6(1)), a plugin called bUnwarpJ was used to align the images, which is crucial for image subtraction operations that were used. The algorithm performs a simultaneous deformation in two images to make one look as similar as possible to each other.

A binarization procedure was performed (Figure 4.6(2)) using different automatic thresholds for both images. Examples are shown in Figure 4.7. Due to the differences in illumination between one experiment and another, different thresholds were tested; Default, Huang and Triangle. The choice of the best threshold for each test was made by comparing the number of lamellae obtained with the software with manual counting for all tests at different times. 

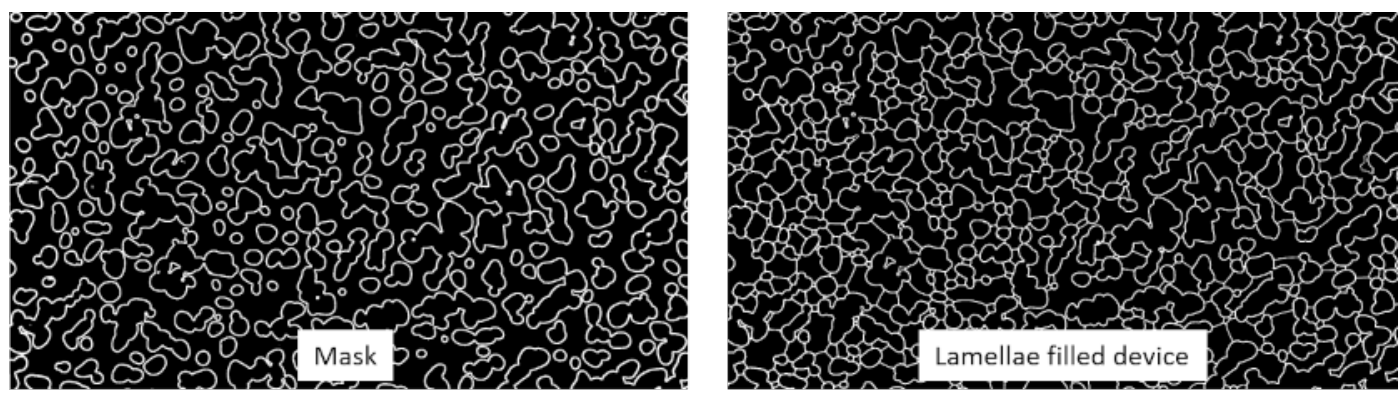

Figure 4.7: Binary images

The next step, Figure 4.6(3), refers to the removal of spurious particles, smaller than 100 pixels.

Following, a logical operation was performed between the binary, aligned images to remove the solid matrix and isolate the lamellae structures (Figure 4.6(4)). Again, spurious particles, now smaller than 10 pixels, were removed after step 4. This step is described in item 5 of Figure 4.6.

Figure 4.8(A) shows an example of the result of such operations. The following step is called Skeletonize, in which pixels were repeatably removed from the edges of objects until they are reduced to single-pixel-wide shapes (Figure 4.6(6)). In the skeletonized images, the nodes defined as the junction of two or more lamellae are identified and removed (Figure 4.6(7)). Nodes are marked by red circles in Figure 4.8(B). The removal of the nodes separates lamellae that are connected into different objects.
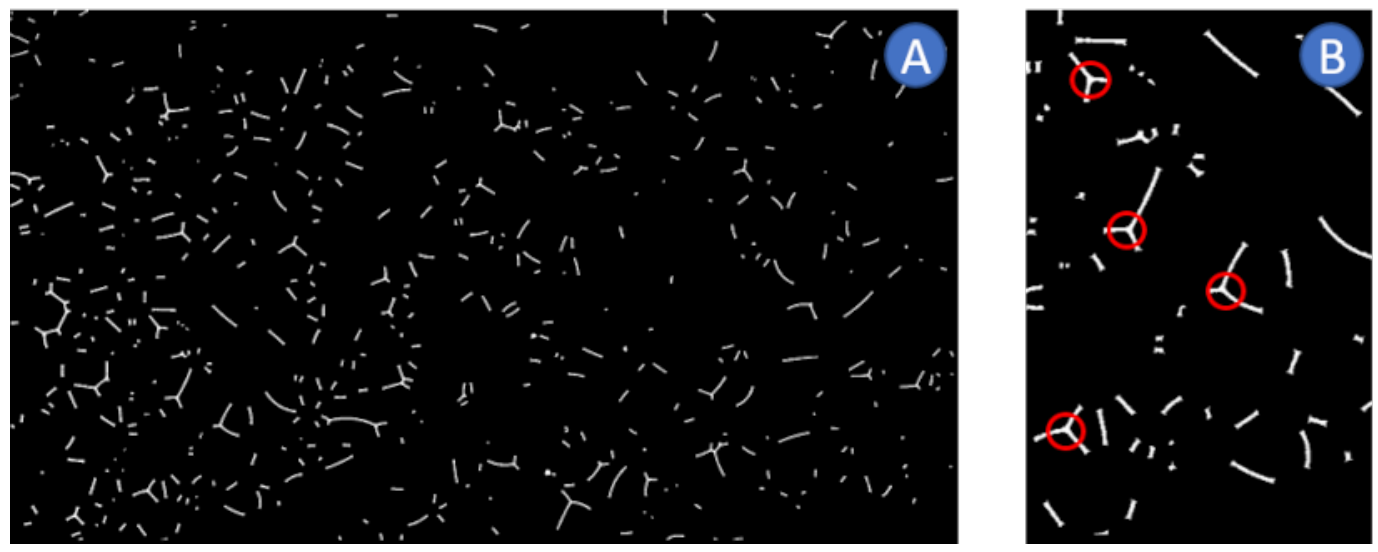

Figure 4.8: A)Lamellae identified by subtraction B)Nodes

A dilation operation was performed to recover the thickness lost by the lamella during the skeletonization step (Figure 4.6(8)). Another logical operation was performed and obtain lamellae without the nodes (Figure 4.6(9)). At this moment, each individual object consists of one lamella. 
The number of objects (lamellae) is then quantified (Figure 4.6(10)) for each frame and it is possible to build a graph with the evolution of the number of lamellae present in the observed area throughout the experiment.

Figure 4.9 shows an example of an image with the counted lamellae, marked in red, over an image of the porous medium.

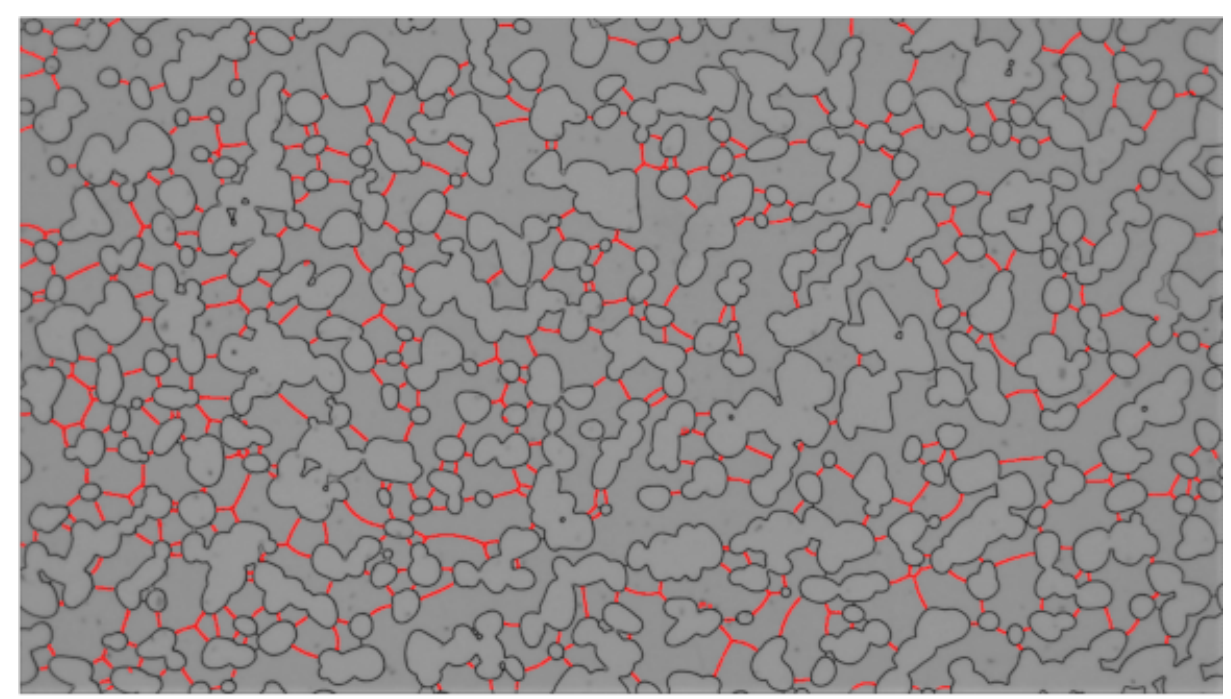

Figure 4.9: Lamellae marked in red

\section{3}

\section{Results}

The idea of the drainage experiments is to analyze the effect of surfactant concentration on foam formation. They were carried out as described in section 4.1 and monitored with a pressure transducer and a camera.

\subsection{1}

\section{Gas displacing pure water}

As a base case, the first experiment consisted of pure water displacement by gas injection. The evolution of the inlet pressure for an injection flow rate of $\mathrm{q}=1 \mathrm{ml} / \mathrm{h}$ is presented in Figure 4.10. The inlet pressure rises in the initial stages of the displacement process to overcome the capillary pressure and then falls as the lower viscosity phase (gas) displaces the higher viscosity phase (water). The inlet pressure reaches steady state after approximately 20 min (145 pore volumes). The phase distribution after steady state is shown in Figure 4.11. The red fluid is water and the gray fluid is gas. Because of the very high viscosity ratio between the phases, the injected gas forms a preferential path that percolates the porous medium. The amount of water remaining in the micromodel after gas injection was approximately $70 \%$. 


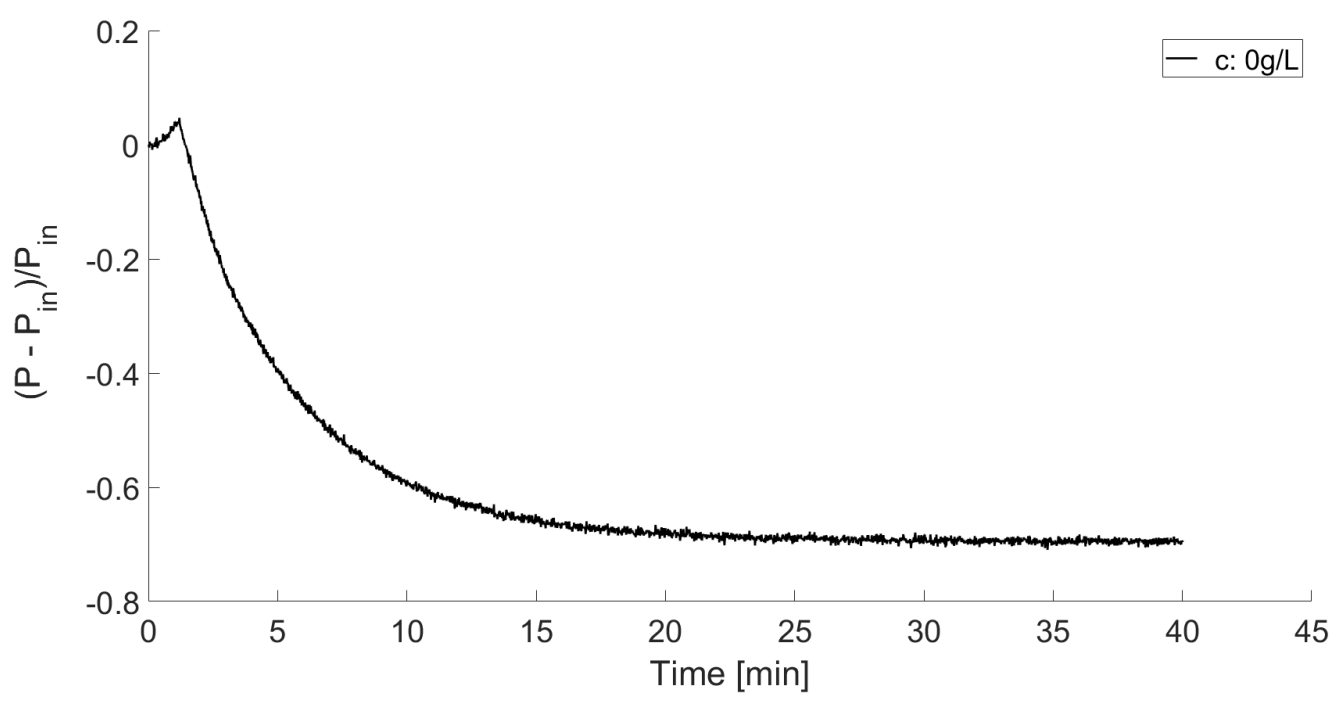

Figure 4.10: Normalized pressure behavior during gas injection - no surfactant added

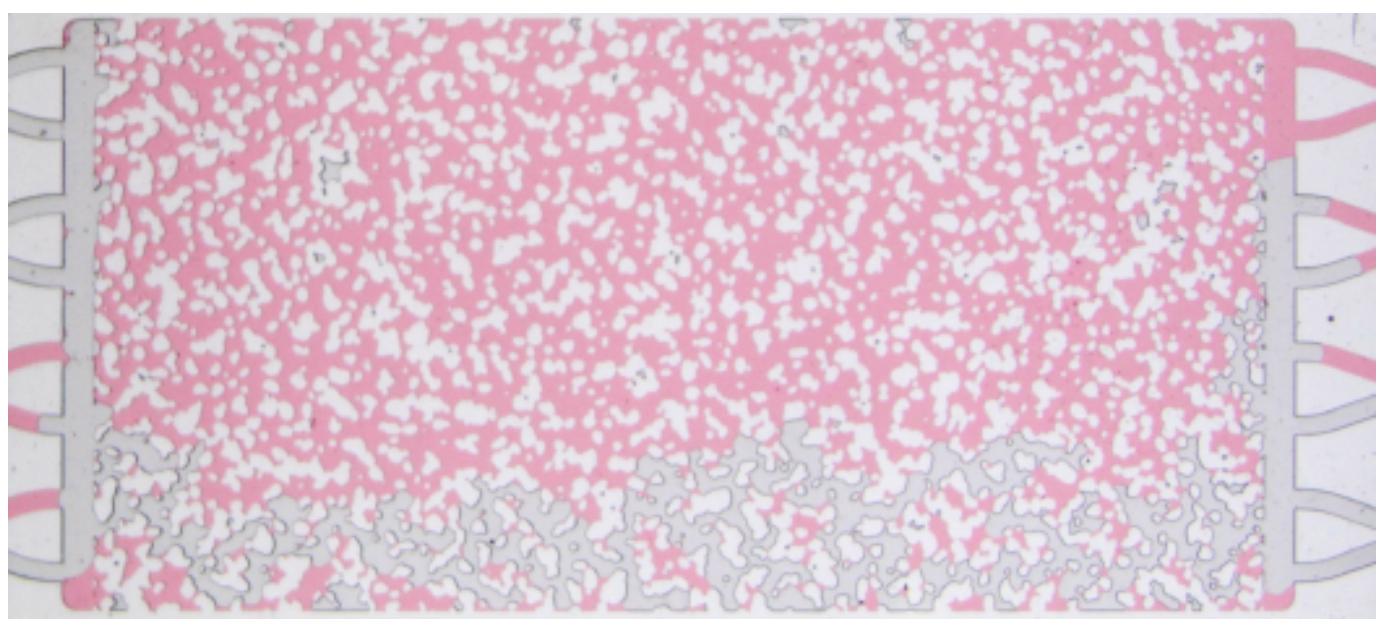

Figure 4.11: Phase distribution after gas injection - no surfactant added

Foam is not formed in this experiment because of the high surface tension and because there is no Marangoni effect stabilizing the liquid films.

\subsection{2}

\section{Foam formation in the surfactant solution displacement experiments}

During displacement of surfactant solution by gas, it was possible to observe the different mechanisms associated with foam formation and destruction.

Evidences of these mechanisms are presented next:

Leave behind mechanism was observed in the glass micromodel at the beginning of all experiments when surfactant was present in the aqueous phase. In Figure 4.12, a yellow bubble invades a pore body saturated with surfactant 
solution, frames A and B, forming two new lamellae, shown as red lines in frame C.

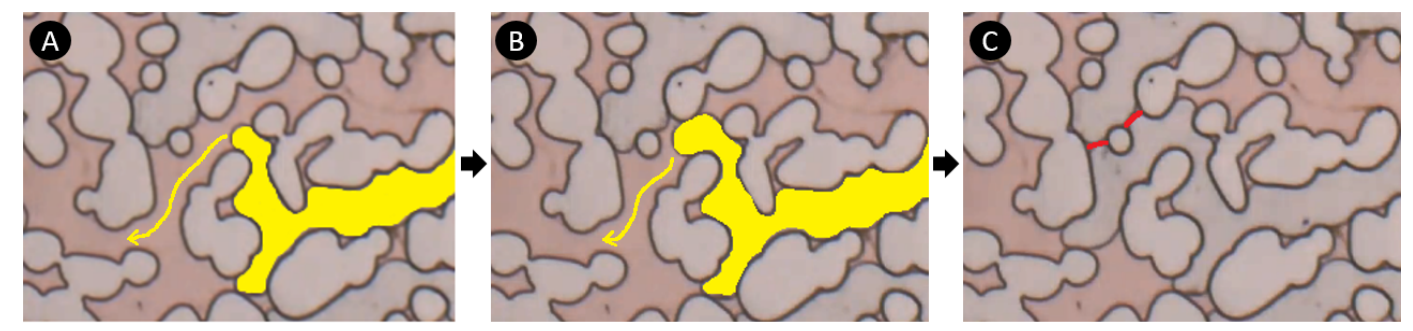

Figure 4.12: Leave behind - A) adjacent pore bodies saturated with surfactant solution - B) gas invasion - C) leave-behind lamellae

Another foam formation mechanism identified in the porous medium was Lamella division. Figure 4.13 shows a digitally colored red lamella flowing past a solid grain and being divided into two new lamellae (blue and green).

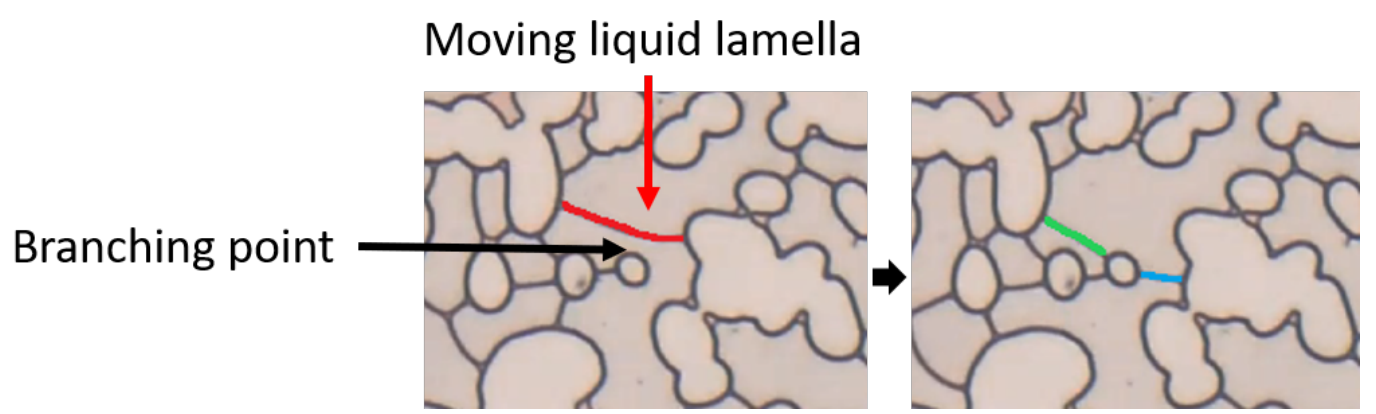

Figure 4.13: Lamella division

During the tests, it was also possible to observe the Snap-off phenomenon. Figure 4.14 shows a bubble (red) flowing through a pore throat and splitting into two bubbles, a new bubble (blue) and the original bubble now with a smaller size (green).
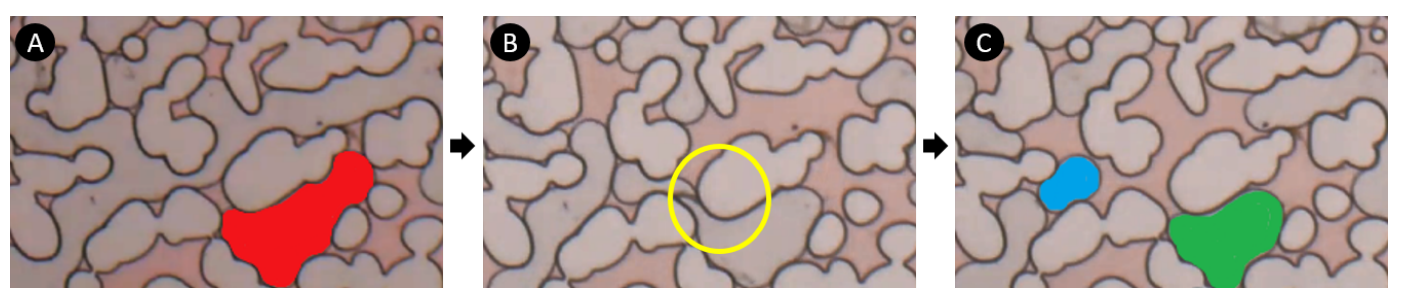

Figure 4.14: Snap-off - A original bubble (red) - B gas-filled pore throat - C new bubble (blue) created after snap-off

An example of Pinch off is presented in Figure 4.15. A bubble, digitally colored in yellow, squeezes another bubble (red) forming a third bubble, colored in blue. 


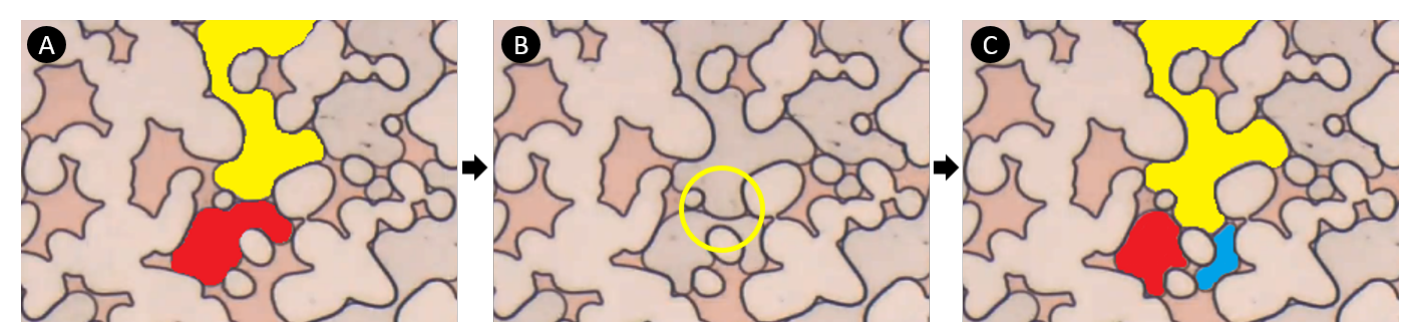

Figure 4.15: Pinch-off - A - bubbles enter pore throat - B red bubble squeezed by yellow bubble - C bubble pinched add lamellae (blue)

Concerning the foam destruction mechanisms, Coarsening was also observed in the microfluidic device. The gas diffuses from the smaller bubble towards larger bubbles and a foam cell collapses, as shown in Figure 4.16.
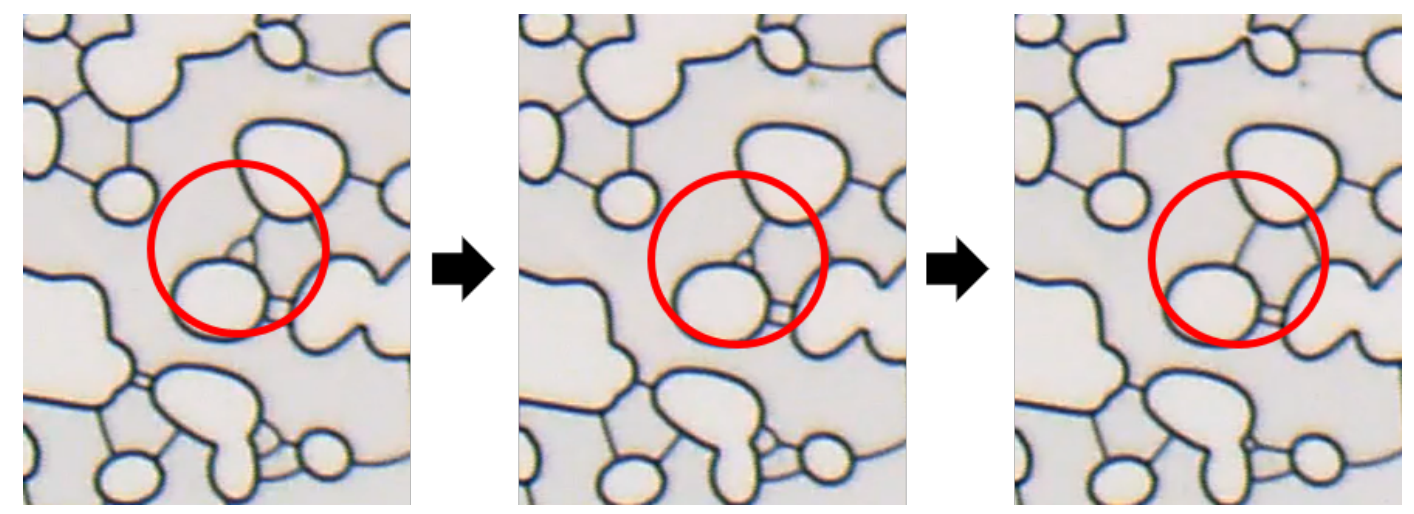

Figure 4.16: A three-sided cell marked with a red circle disappeared due to diffusion

Adding surfactant to the water completely changes the gas distribution inside the porous medium. At a surfactant concentration of $\mathrm{c}=0.235 \mathrm{~g} / \mathrm{L}$, below the CMC, a few liquid films are generated mostly by leave-behind mechanism. This mechanism creates lamellae parallel to the flow direction that provide little resistance to gas flow.

The evolution of the inlet pressure at an injection flow rate of $\mathrm{q}=1 \mathrm{ml} / \mathrm{h}$ is presented in Figure 4.17. As a basis of comparison, the inlet pressure for the pure water experiment is also shown. At this concentration, $\mathrm{c}=0.235 \mathrm{~g} / \mathrm{L}$, the maximum pressure is slightly lower than the maximum pressure obtained with pure water because of the lower interfacial tension and consequently lower capillary pressure.

The inlet pressure during surfactant solution displacement by gas stabilizes at a higher value than that observed in the pure water experiment. The higher values can be associated with the added resistance of the gas flow associated with the lamellae that were formed. 


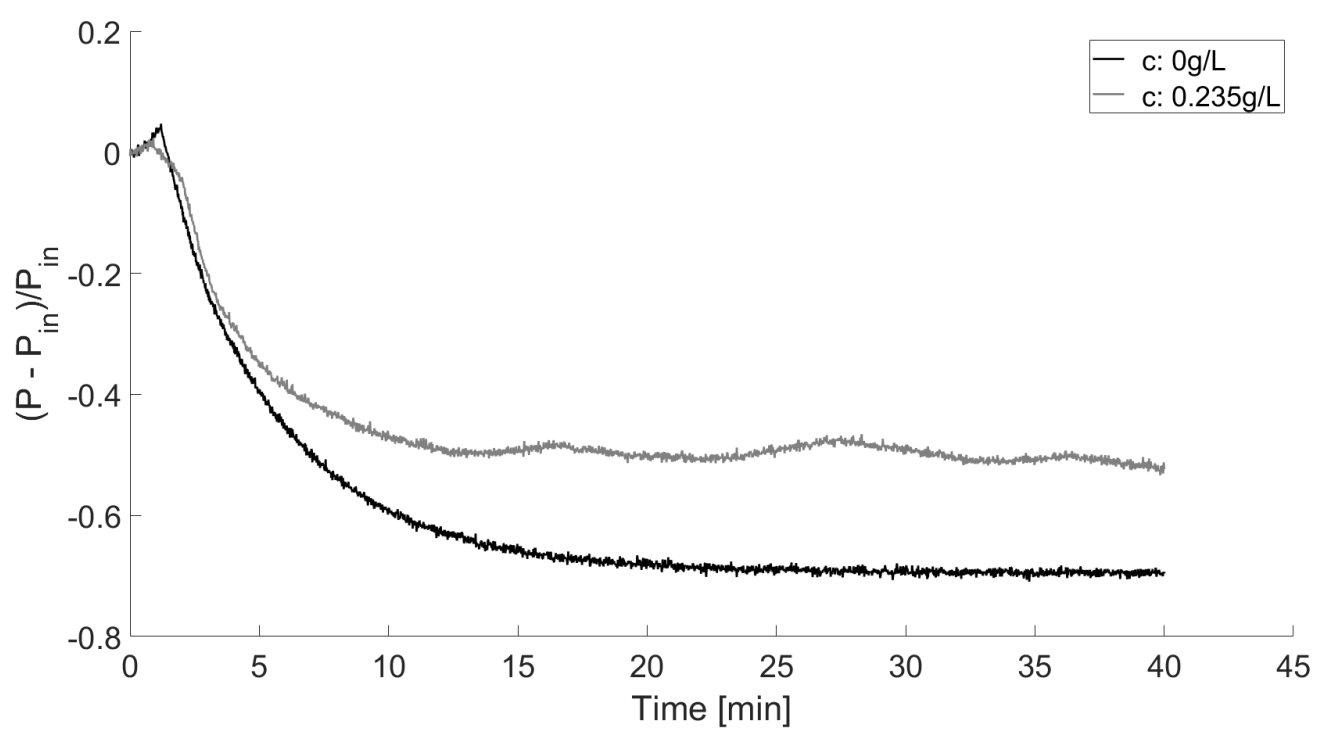

Figure 4.17: Normalized pressure behavior during gas injection

The phase distribution at steady state is shown in Figure 4.18. The aqueous phase saturation (surfactant solution $\mathrm{c}=0.235 \mathrm{~g} / \mathrm{L}$ ) is much lower $(45 \%)$ than in the pure water experiment (70\%). The mobility reduction of the gas phase led to flow diverging. Two main gas preferential paths can be observed in the figure.

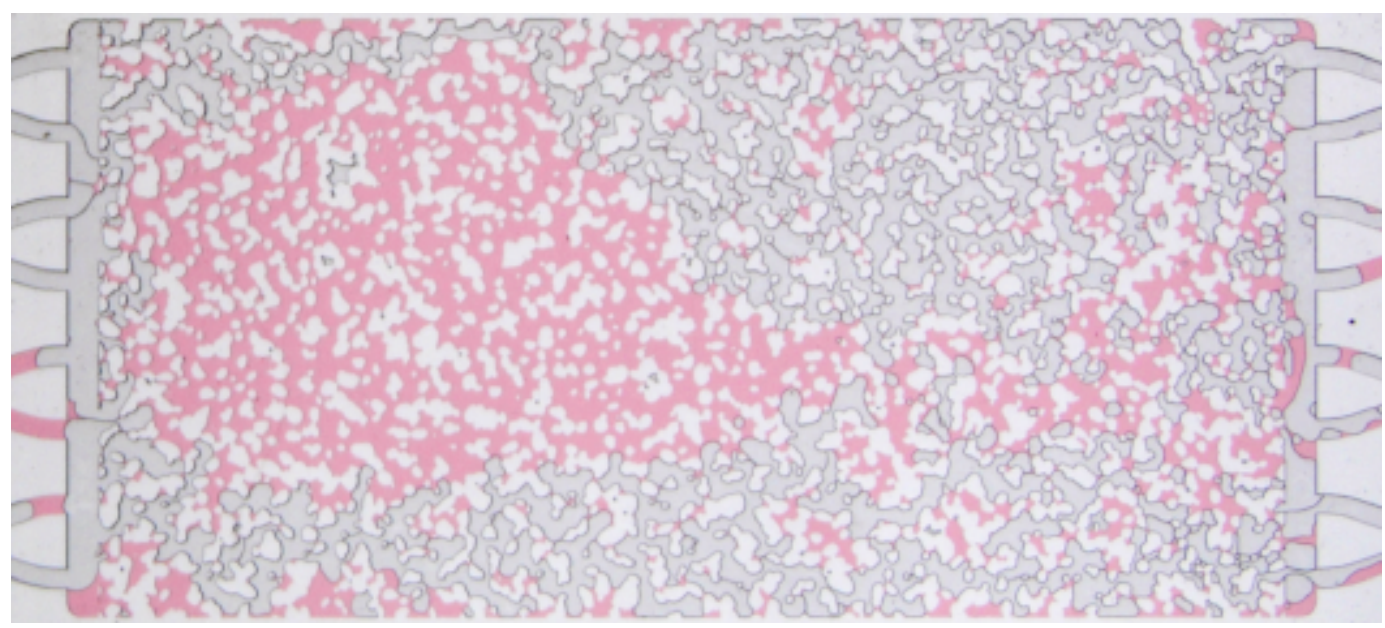

Figure 4.18: Phase distribution after gas injection - surfactant concentration below $\mathrm{CMC}(\mathrm{c}=0.235 \mathrm{~g} / \mathrm{L})$

At a concentration of $\mathrm{c}=0.47 \mathrm{~g} / \mathrm{L}$ the gas flows discontinuously and the flow resistance of the liquid films formed contributes to an increase in the inlet pressure. At this condition, foam is formed not only by the leave-behind mechanism, but also by snap-off, lamellae division and pinch-off.

Figure 4.19 presents the time evolution of the inlet pressure at $\mathrm{q}=1 \mathrm{ml} / \mathrm{h}$ for different values of the surfactant concentrations below the CMC. For pure 
water and low surfactant concentration close to $0.1 \times \mathrm{CMC}(\mathrm{c}=0.235 \mathrm{~g} / \mathrm{L})$, which was presented in Figure 4.17 and repeated in the plot as reference, the inlet pressure rises for a very short time and then falls quickly, until stabilizing at a value lower than the inlet pressure at the moment gas enters the porous medium.

In the displacement of a surfactant solution with higher concentrations, the inlet pressure increases for a long time, reaches a maximum value and falls before reaching a steady-state plateau. Foam formation lasts for a long time. As lamellae are formed, the flow resistance increases, leading to higher inlet pressures. After a while, most of the water has been displaced and the lamellae become thin and break, leading to a percolated path for the gas flow. This corresponds to the sharp drop in the inlet pressure. The critical thickness for lamellae breakups reduces as the surfactant concentration rises, which explains the delay of the pressure decrease.

The time and value of the maximum pressure rises with the surfactant concentration. At $\mathrm{c}=0.47 \mathrm{~g} / \mathrm{L}(\approx 0.2 \times \mathrm{CMC})$, the maximum pressure occurs at $\mathrm{t} \approx 20 \mathrm{~min}(145$ pore volumes $) ;$ at $\mathrm{c}=1.55 \mathrm{~g} / \mathrm{L}(\approx 0.7 \times \mathrm{CMC})$, the maximum pressure occurs at $\mathrm{t} \approx 40 \mathrm{~min}$ (290 pore volumes).

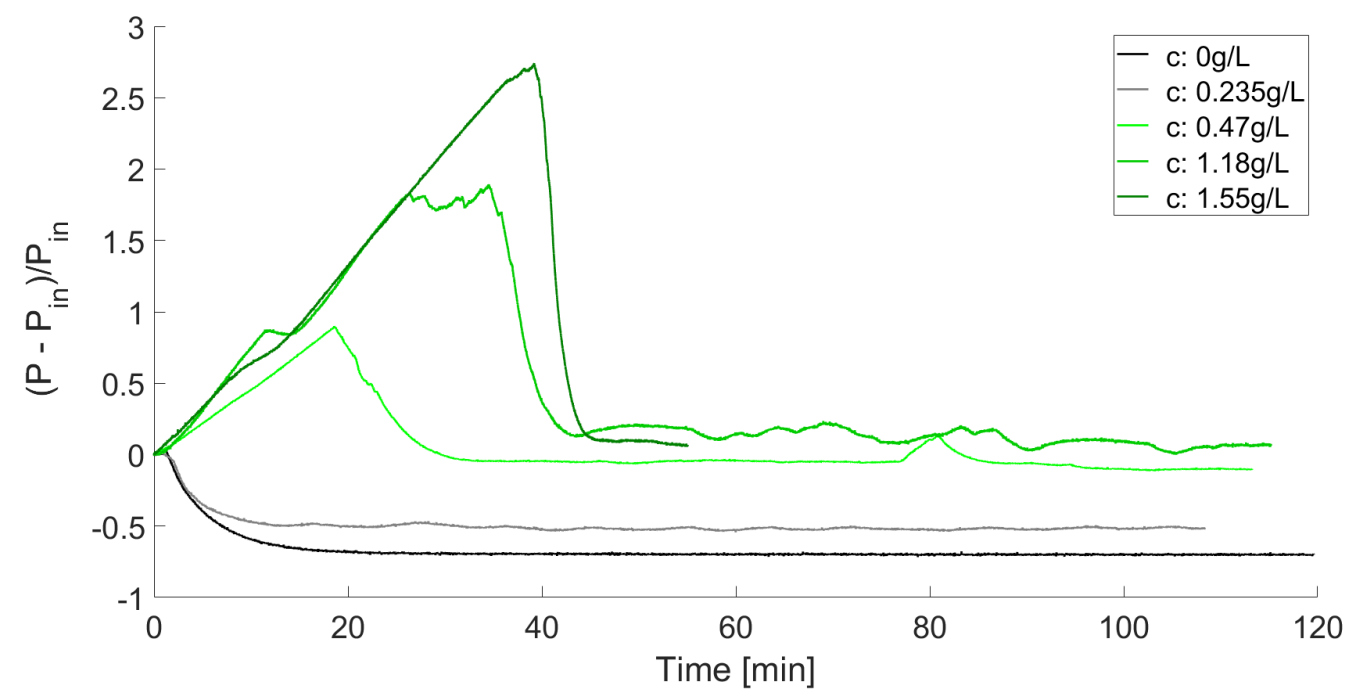

Figure 4.19: Normalized pressure behavior during gas injection - surfactant concentrations below the CMC

The trend continues as the surfactant concentration increases even further. Figure 4.20 presents the inlet pressure evolution at $\mathrm{q}=1 \mathrm{ml} / \mathrm{h}$ up to $\mathrm{c}=$ $15.5 \mathrm{~g} / \mathrm{L}(\approx 6.5 \times \mathrm{CMC})$. The change in behavior above the $\mathrm{CMC}$ is clear, it is possible to observe two different plateaus in the pressure curves, one below the CMC and the other above. The value of the maximum pressure and the 
time it occurs still rises with surfactant concentration. At $\mathrm{c}=15.5 \mathrm{~g} / \mathrm{L}(\approx 6.5$ $\times \mathrm{CMC}$ ), the maximum pressure occurs at $\mathrm{t} \approx 100 \mathrm{~min}$ (730 pore volumes).

It is interesting to note that the slope of the inlet pressure data during the foam formation process is approximately the same for all the surfactant concentrations explored.

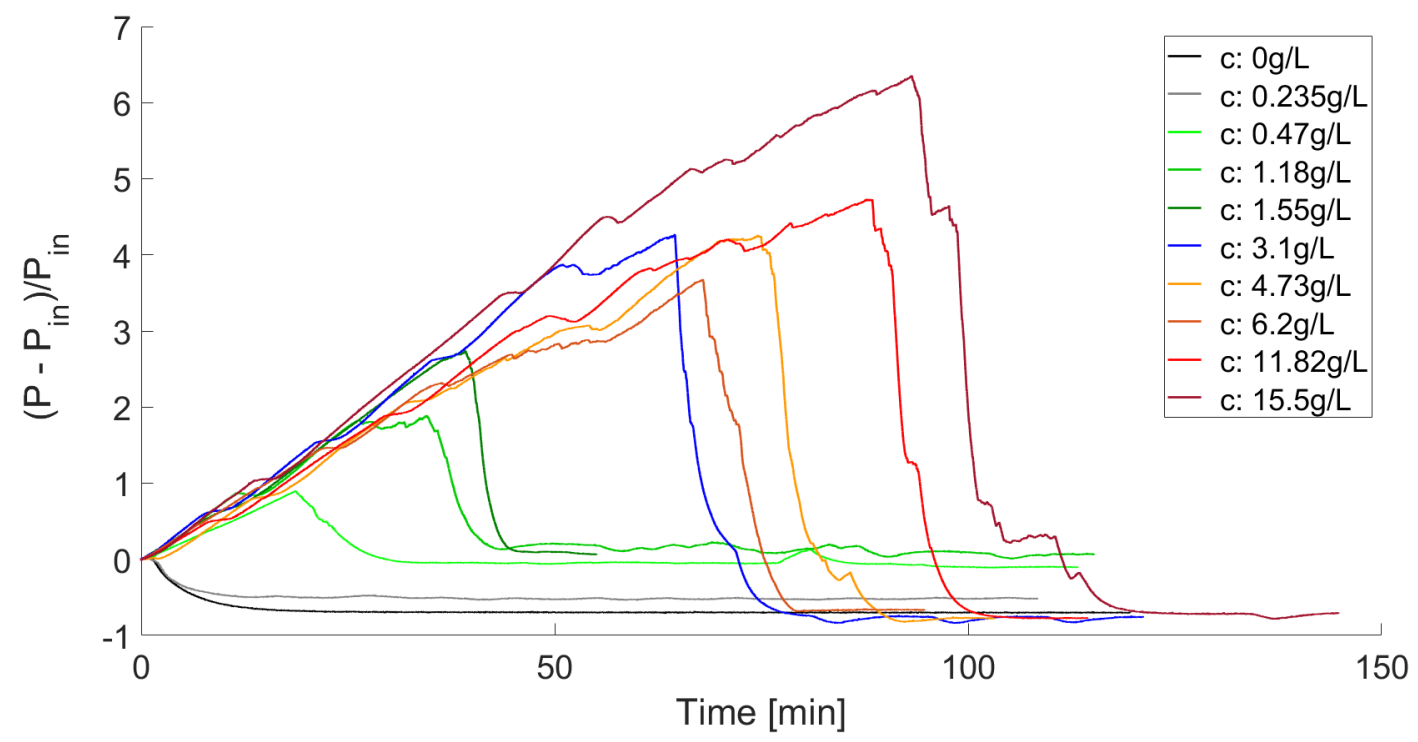

Figure 4.20: Normalized pressure behavior during gas injection

The normalized maximum pressure is shown in Figure 4.21 and the time it occurs in Figure 4.22 as a function of surfactant concentration. It is possible to notice a gradual increase in the normalized maximum pressure with increasing surfactant concentration (Figure 4.21). A sharp increase is observed below the CMC. The maximum pressure stabilizes near the CMC and then starts to increase again. Same trend was observed for the time when maximum pressure was reached during gas injection (Figure 4.22). 


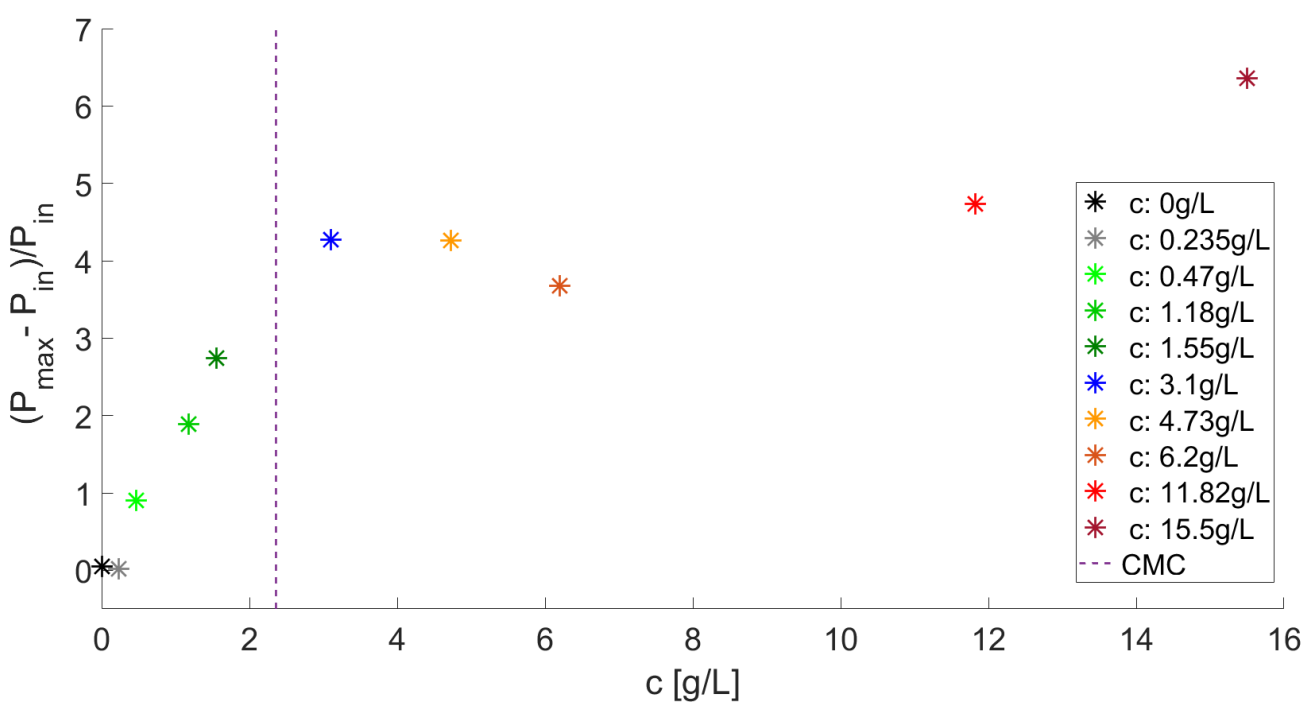

Figure 4.21: Maximum pressure for different concentrations

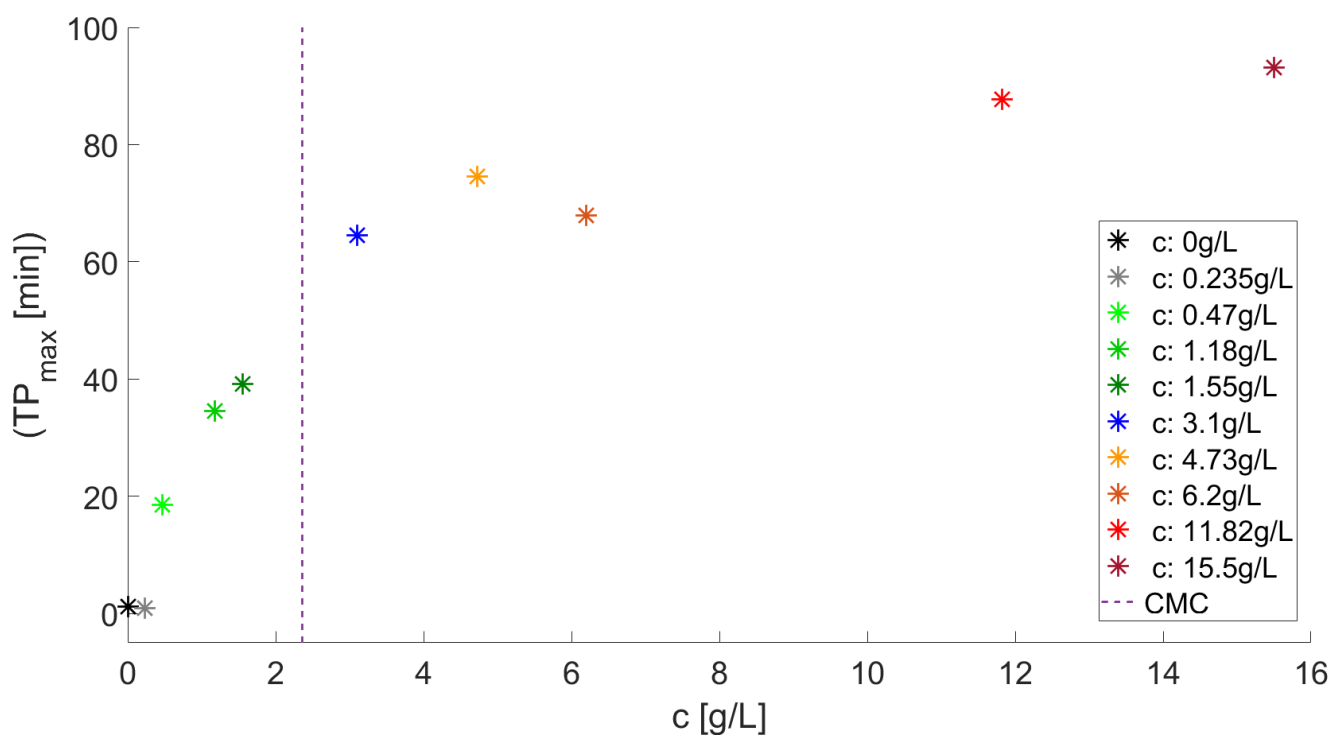

Figure 4.22: Time when the maximum pressure was reached for different concentrations

Foam strength can be determined by the inlet pressure measurements. Stronger foam, has a higher flow resistance that leads to higher inlet pressure.

Increasing the surfactant concentration to well above the CMC dramatically increases the pressure drop. Generating a more effective foam from a continuous gas foam requires mobilizing these lamellae so that they can multiply by lamella division and by repeated snap-off at the unoccupied pore throats.

According to Aronson [37], strong foam in porous media with large flow resistance is produced by surfactant solutions with high repulsive disjoining pressures. The reported behavior was also observed in the experiments pre- 
sented here. At higher concentrations (well above the CMC) and high disjoining pressures, more lamellae can be created and a higher resistance is imposed to the gas flow represented by the increase in the pressure gradient.

Rossen et al. [57] studied the ability of foam to reduce gas mobility after a long period of gas injection. In a field test, one slug of surfactant solution was injected followed by months of gas injection, they concluded that foam continued to reduce mobility by a modest amount even after long injection of gas. However, foam did weaken progressively as it dried out. They also commented that foam models assuming that foam remains strong at irreducible water saturation significantly overestimates foam effectiveness over extended periods of time.

It is important to note that the steady-state inlet pressure at the end of gas injection varies with the surfactant solution concentration. The results are summarized in Figure 4.23. In the displacement of pure water, the dimensionless final inlet pressure is approximately $\bar{P}(t)=\left(P(t)-P_{\text {in }}\right) / P_{\text {in }} \approx$ -0.8 . The steady state inlet pressure rises when surfactant concentration is near the CMC, reaching $\bar{P}(t \rightarrow \infty) \simeq 0$. At higher surfactant concentration, the final dimensionless pressure is lower, close to $\bar{P} \approx-0.8$. At very high surfactant concentration, the formed lamellae are thinner and easier to be displaced, leading to a smaller final inlet pressure.

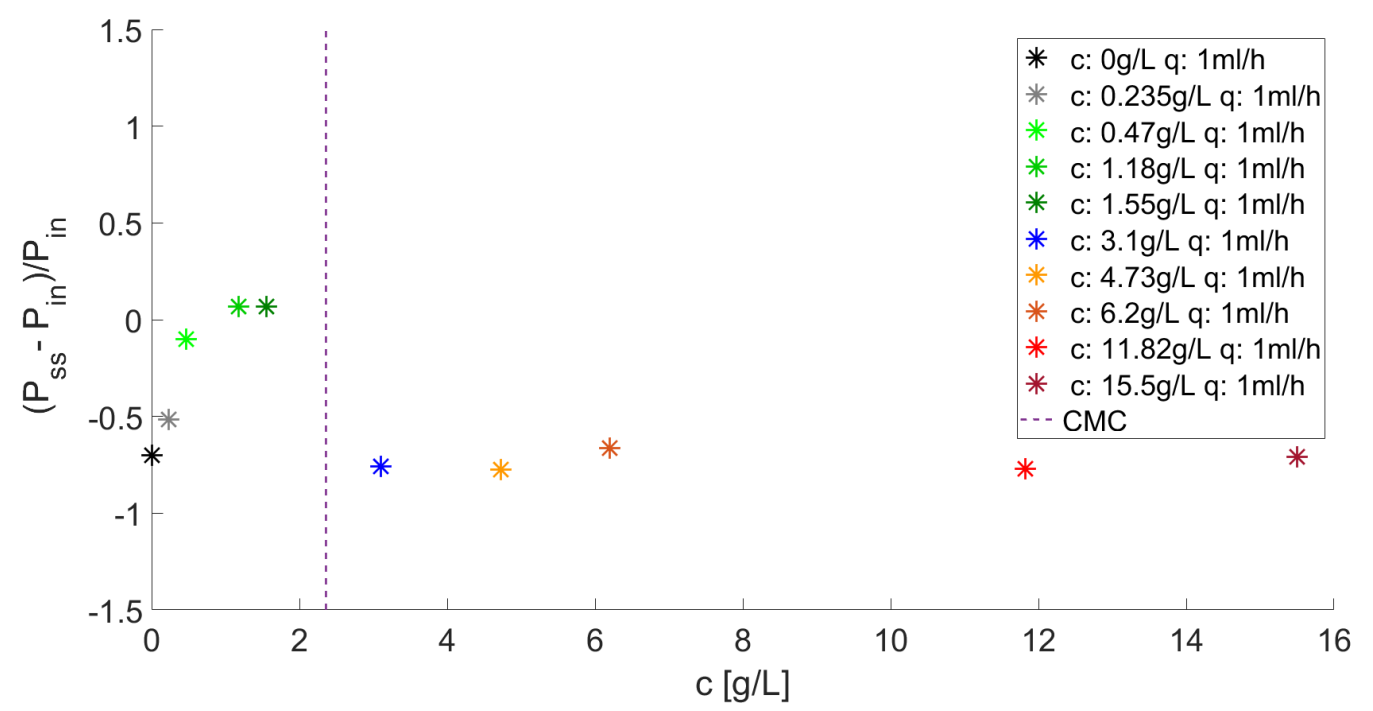

Figure 4.23: Pressure at steady state condition for different concentrations

At high enough surfactant concentration, as the surfactant solution is displaced by the gas phase, pore-spanning lamellae are formed in the early stages of the process. Because of the relatively high aqueous phase saturation, the pore space is occupied either by a wet foam or a bubbly liquid, as shown in Figure 4.24(A), which presents an image of the downstream part of the porous 
medium at $\mathrm{c}=15.5 \mathrm{~g} / \mathrm{L}$ in the early stages of the experiment. As time passes and the liquid phase is forced out, an evolution towards a dry foam can be observed, as shown in Figure 4.24(B), which shows the phase distribution at later stages of the displacement process.

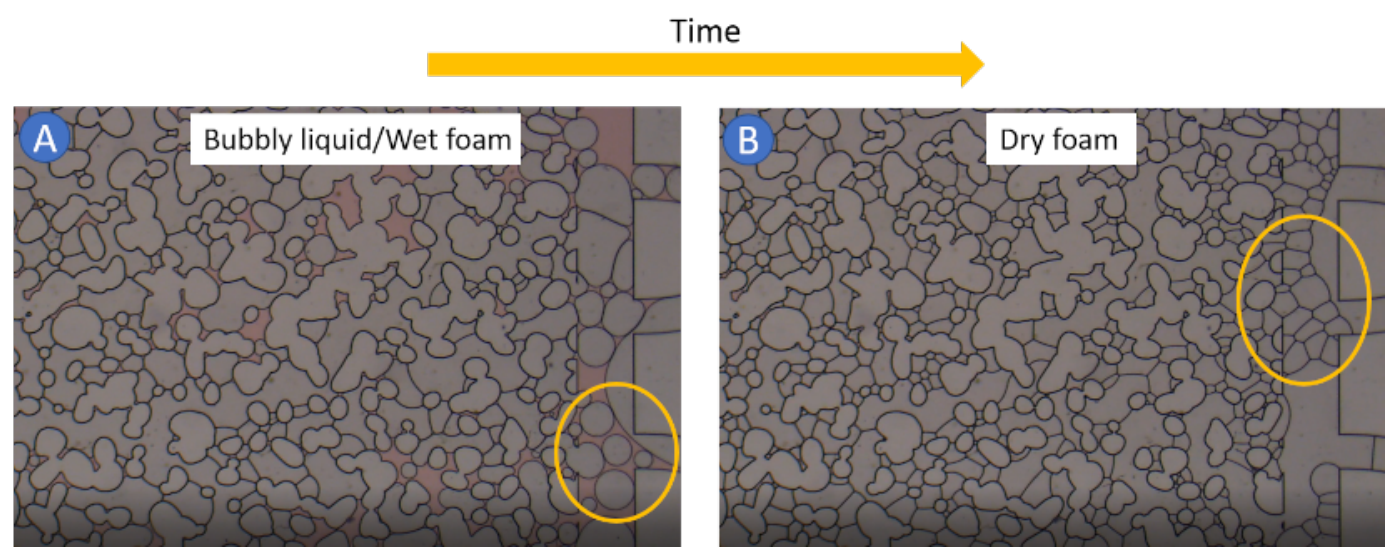

Figure 4.24: Liquid fraction at the beginning of the experiment (A) and at the end $(\mathrm{B}) \mathrm{c}=15.5 \mathrm{~g} / \mathrm{L}$

Because of the lower mobility of the gas phase as foam is formed, the remaining water phase saturation at the end of the process falls as the surfactant concentration increases. The phase distribution at steady state for a surfactant concentration of $\mathrm{c}=0.47 \mathrm{~g} / \mathrm{L}$ is presented in Figure 4.25 . The remaining water saturation (marked in red) is much lower $(8 \%)$ than that observed with pure water (70\%) (Figure 4.11) and $\mathrm{c}=0.235 \mathrm{~g} / \mathrm{L}(45 \%)$ (Figure 4.18).

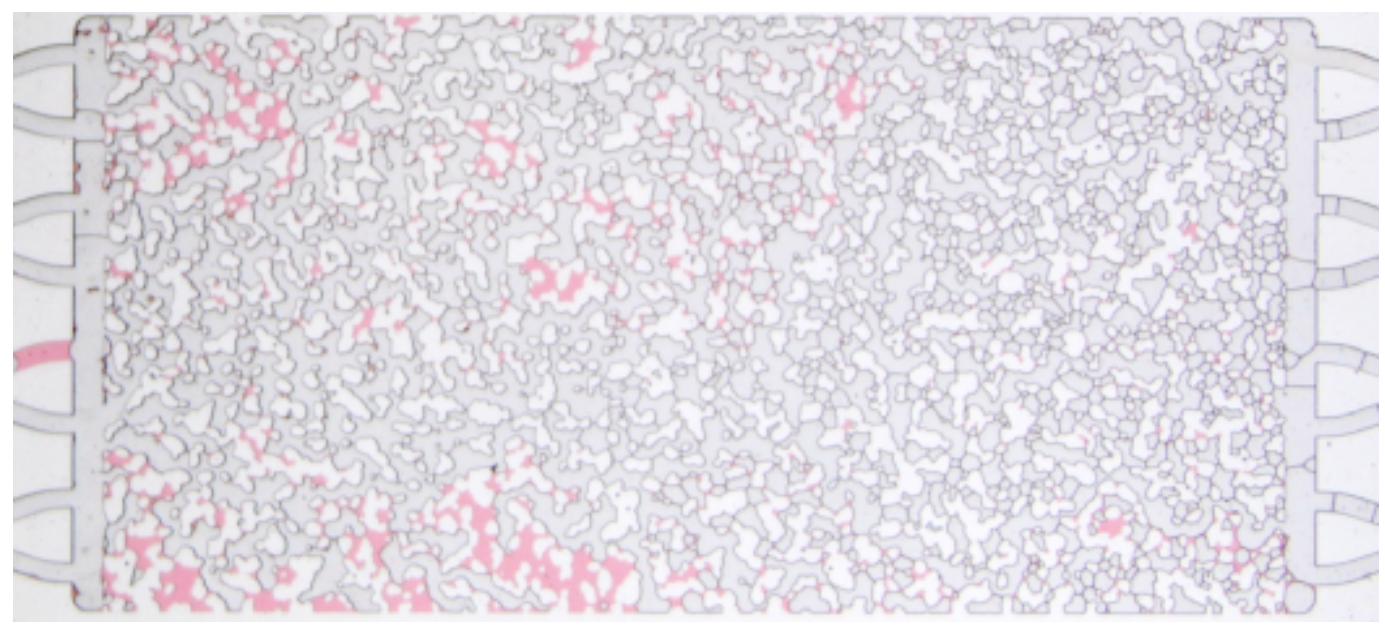

Figure 4.25: Phase distribution after gas injection - surfactant concentration below the $\mathrm{CMC} \mathrm{c}=0.47 \mathrm{~g} / \mathrm{L}$

A large number of lamellae can be observed in the gas occupied pores. With a concentration even higher, water is present in the porous space only as 
thin films, forming the lamellae of the generated foam; as it is clear in Figure $4.26(\mathrm{c}=1.18 \mathrm{~g} / \mathrm{L})$ and Figure $4.27(\mathrm{c}=15.5 \mathrm{~g} / \mathrm{L})$.

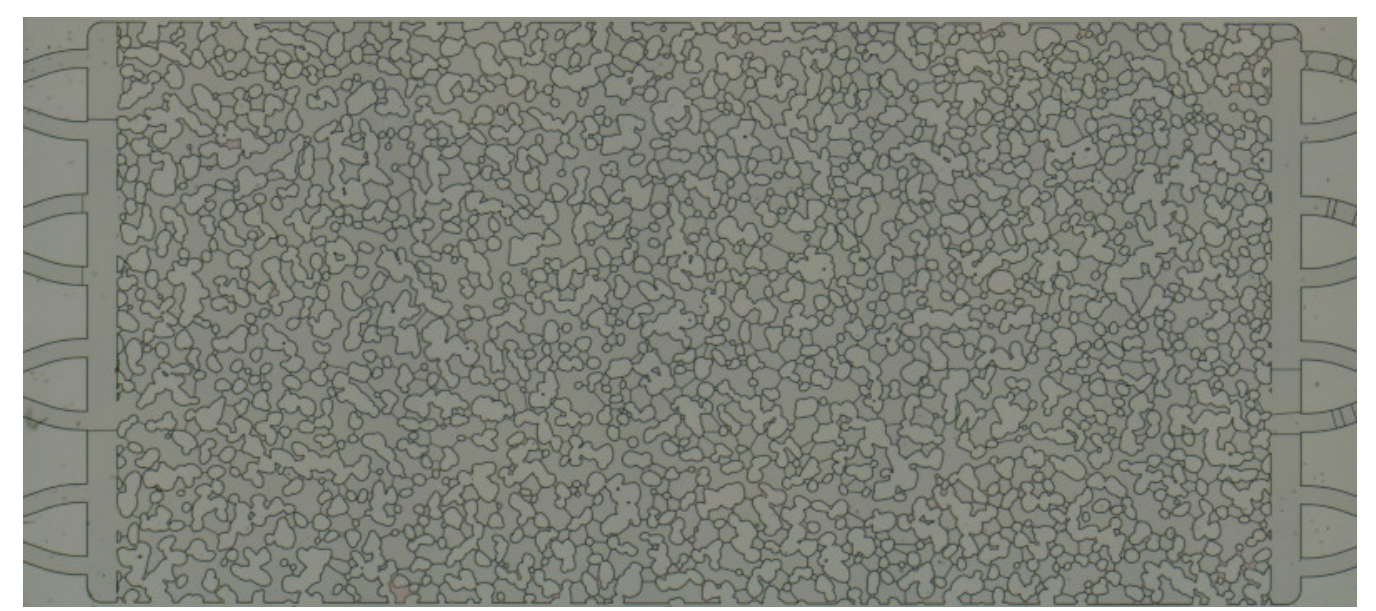

Figure 4.26: Phase distribution after gas injection - surfactant concentration near the $\mathrm{CMC} \mathrm{c}=1.18 \mathrm{~g} / \mathrm{L}$

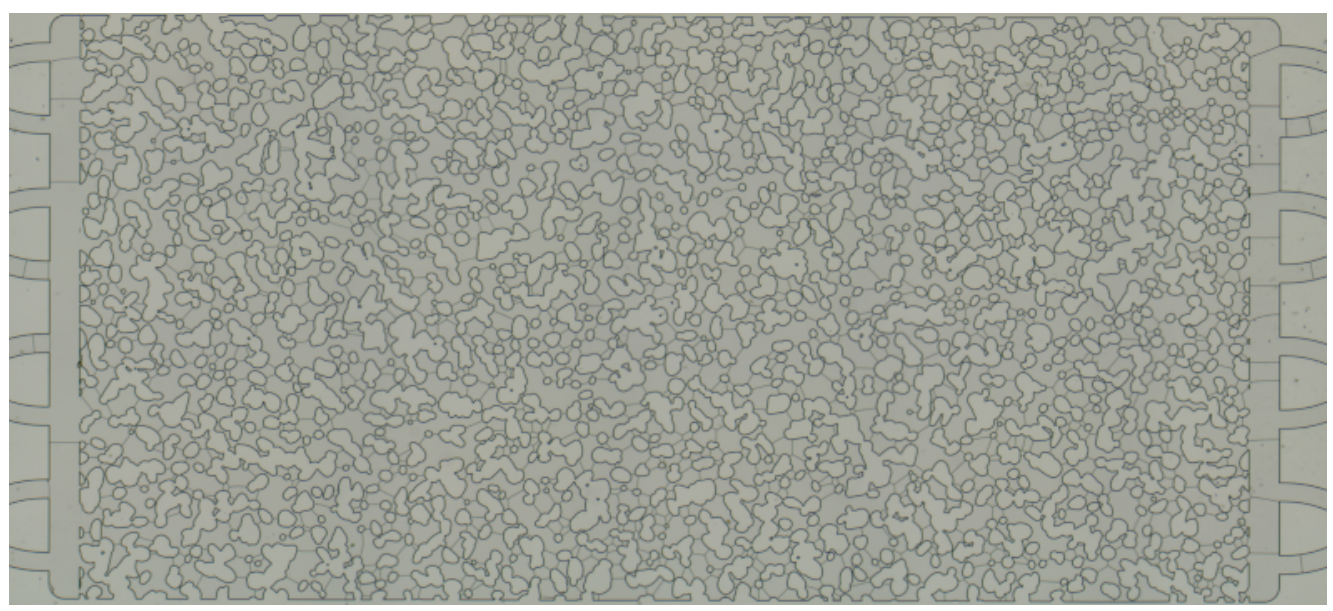

Figure 4.27: Phase distribution after gas injection - surfactant concentration well above the $\mathrm{CMC} \mathrm{c}=15.5 \mathrm{~g} / \mathrm{L}$

The flow resistance and, consequently, the inlet pressure is directly associated with the number of lamellae formed in the pore space. To better understand this relationship, we used image processing to count the number of lamellae inside the window defined in Figure 4.3 as a function of time for the displacement process at surfactant solution near and above CMC. Figure 4.28 presents the evolution of lamellae density (number/unit area) and inlet pressure for $\mathrm{c}=3.1 \mathrm{~g} / \mathrm{L}(\approx 1.3 \times \mathrm{CMC})$ and $\mathrm{q}=1 \mathrm{ml} / \mathrm{h}$. The rate of lamella formation is very high in the early stages of the displacement process, $t<2$ min. After approximately 2 minutes, the lamellae density reaches $10 / \mathrm{mm}^{2}$ and remains approximately the same for a long time, as inlet pressure rises. When the dimensionless inlet pressure is close to $\bar{P}=3$, a fast increase in the number 
of lamellae is observed, reaching a maximum value close to $20 / \mathrm{mm}^{2}$. After the maximum value of the inlet pressure is achieved, the number of lamellae drops together with the inlet pressure. At steady state, both the inlet pressure and lamellae density remain almost constant.

Figure 4.29 presents the evolution of lamellae density at $\mathrm{c}=15.5 \mathrm{~g} / \mathrm{L}(6.5$ $\times$ CMC). The behavior is similar, with regions of sharp increase or decrease in the number of lamellae and regions of more or less constant lamellae density. The decrease in the inlet pressure occurs simultaneously with the fall of the number of lamellae.

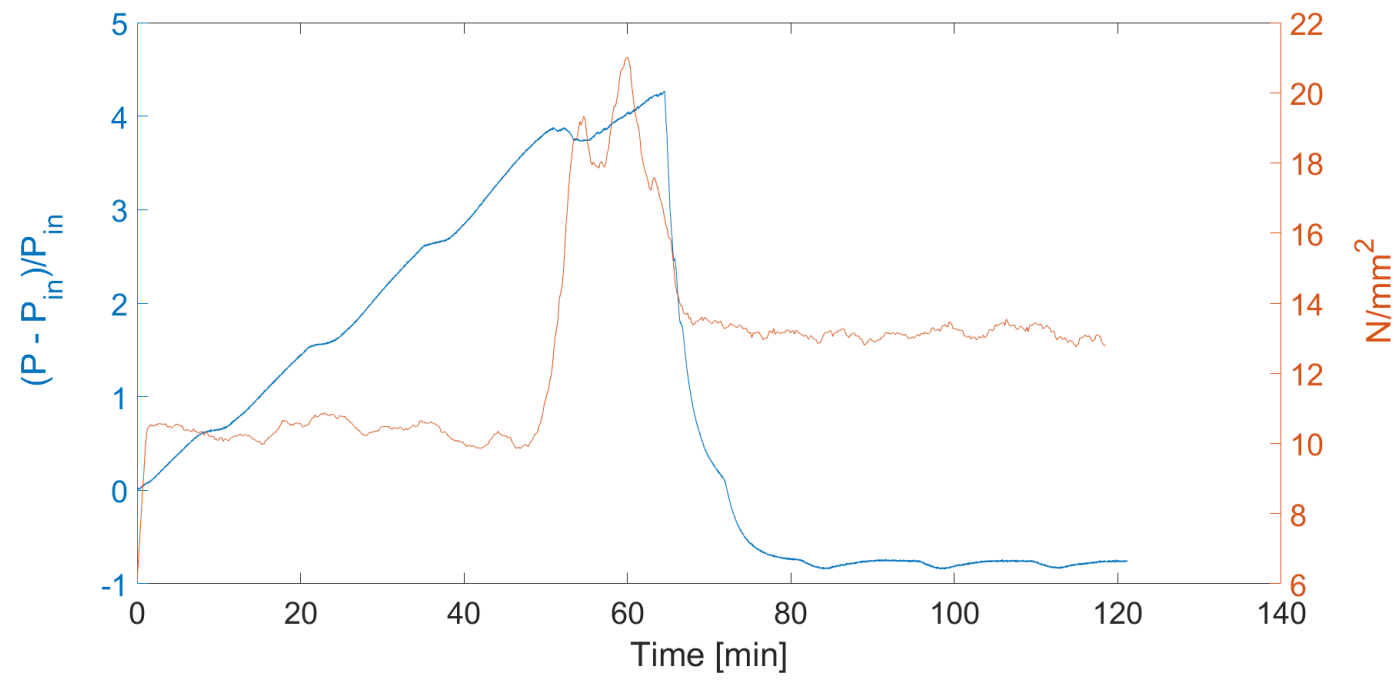

Figure 4.28: Normalized pressure and lamellae number per millimeter square as a function of time (concentration $=3.1 \mathrm{~g} / \mathrm{L}$ and gas flow rate $=1 \mathrm{ml} / \mathrm{h}$ )

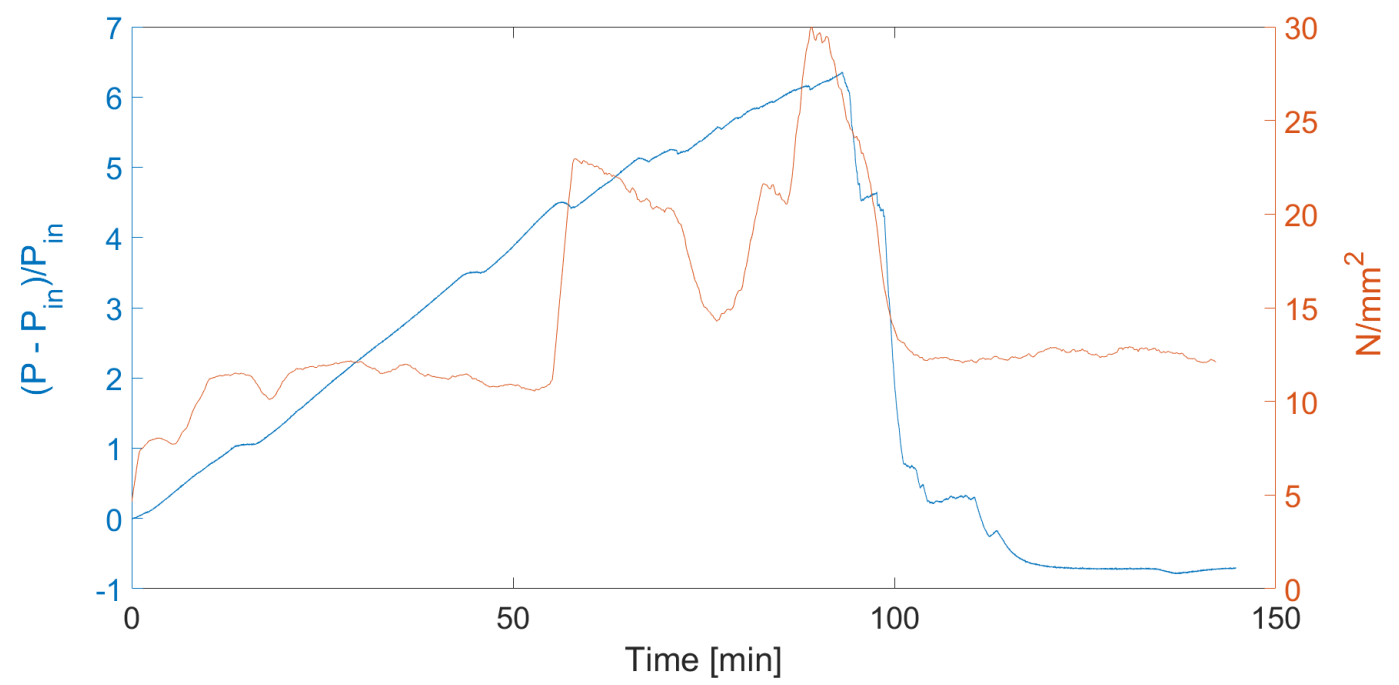

Figure 4.29: Normalized pressure and lamellae number per millimeter square as a function of time (concentration $=15.5 \mathrm{~g} / \mathrm{L}$ and gas flow rate $=1 \mathrm{ml} / \mathrm{h}$ ) 
The evolution of the lamellae density as a function of time for different surfactant concentrations is shown in Figure 4.30. The overall behavior is similar to that presented in Figures 4.28 and 4.29. At steady state, the density was between 10 and $12 / \mathrm{mm}^{2}$ for all experiments, despite the different final inlet pressure.

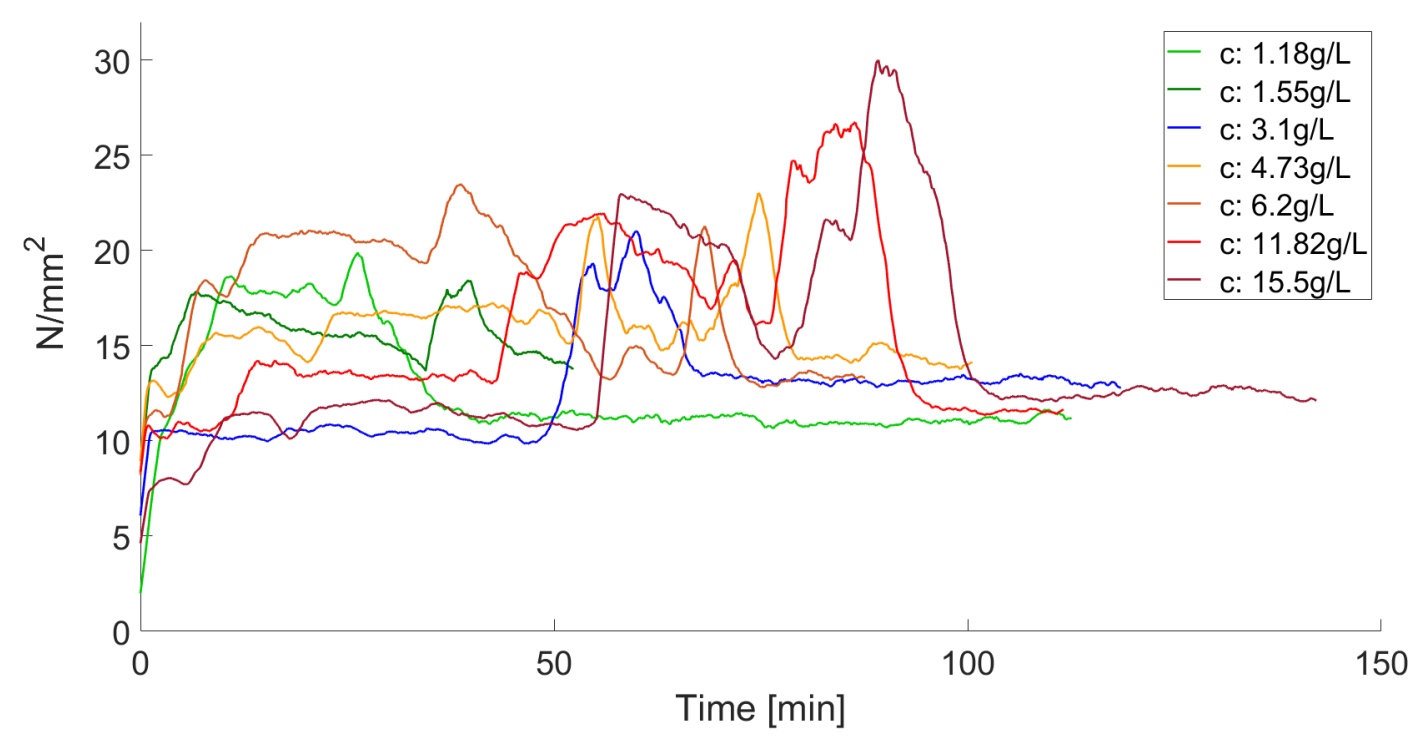

Figure 4.30: Lamellae number per millimeter square vs time

There is always a clear maximum and its value and the time it occurs rises with the surfactant concentration. Figure 4.31 presents the maximum lamellae density as a function of surfactant concentration.

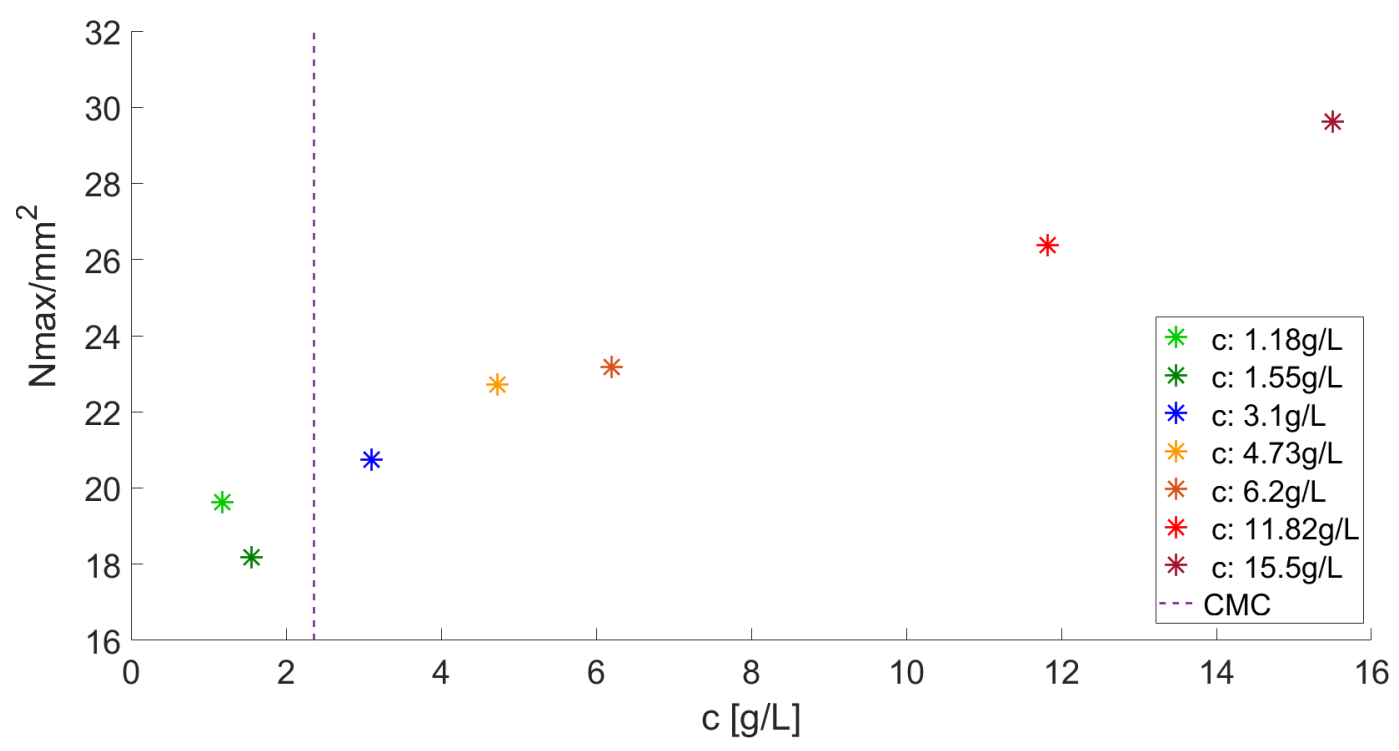

Figure 4.31: Maximum lamellae density vs surfactant solution concentration

The time evolution of the lamellae density was measured inside a region closer to the inlet side of the porous media. In order to verify the spatial 
distribution of lamellae in the porous space, we evaluate the number of lamellae along the microfluidic model after steady state is achieved. The micromodel was divided in four quarters to study this distribution, named as Q1, Q2, Q3 and Q4, numbered from the inlet to the outlet side (Figure 4.32). The region analyzed in the transient regime is located in the Q1 and Q2 regions.

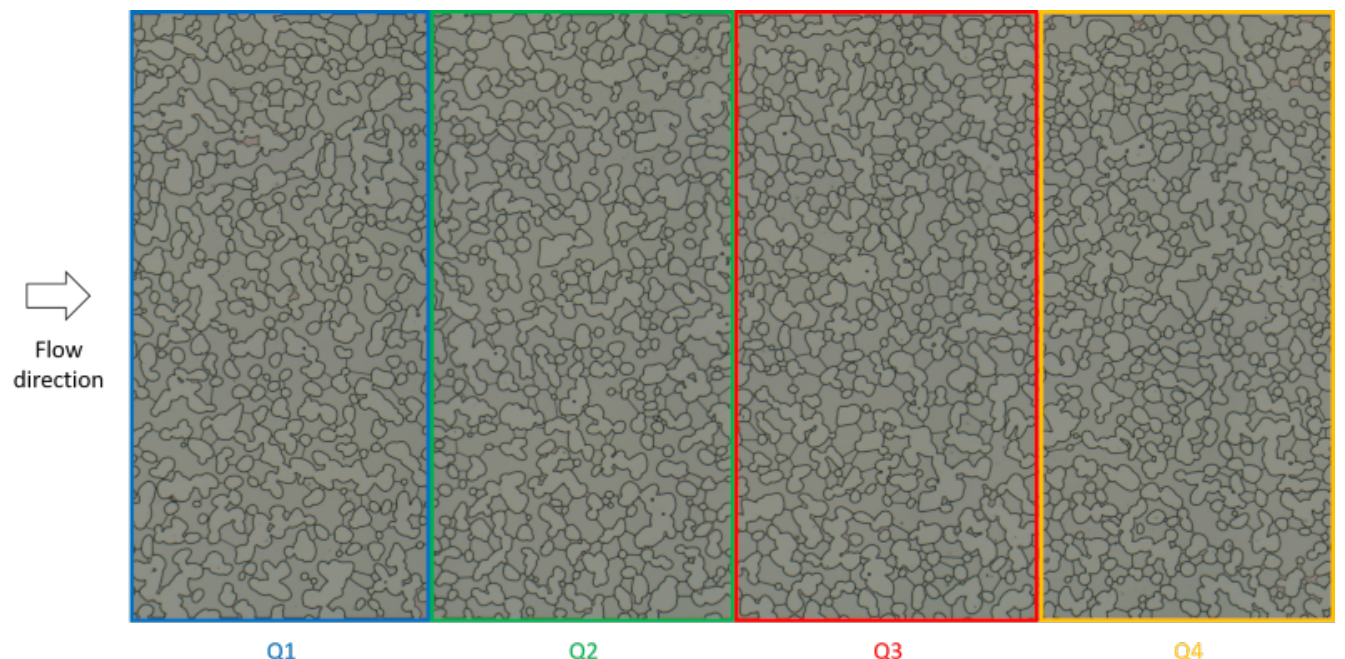

Q2

Q3

Q4

Figure 4.32: Micromodel divided in four quarters (Q1, Q2, Q3 and Q4)

The number of lamellae at the end of the experiment on the entire device was calculated and the values at different surfactant concentration near and above the CMC are shown in Figure 4.33.

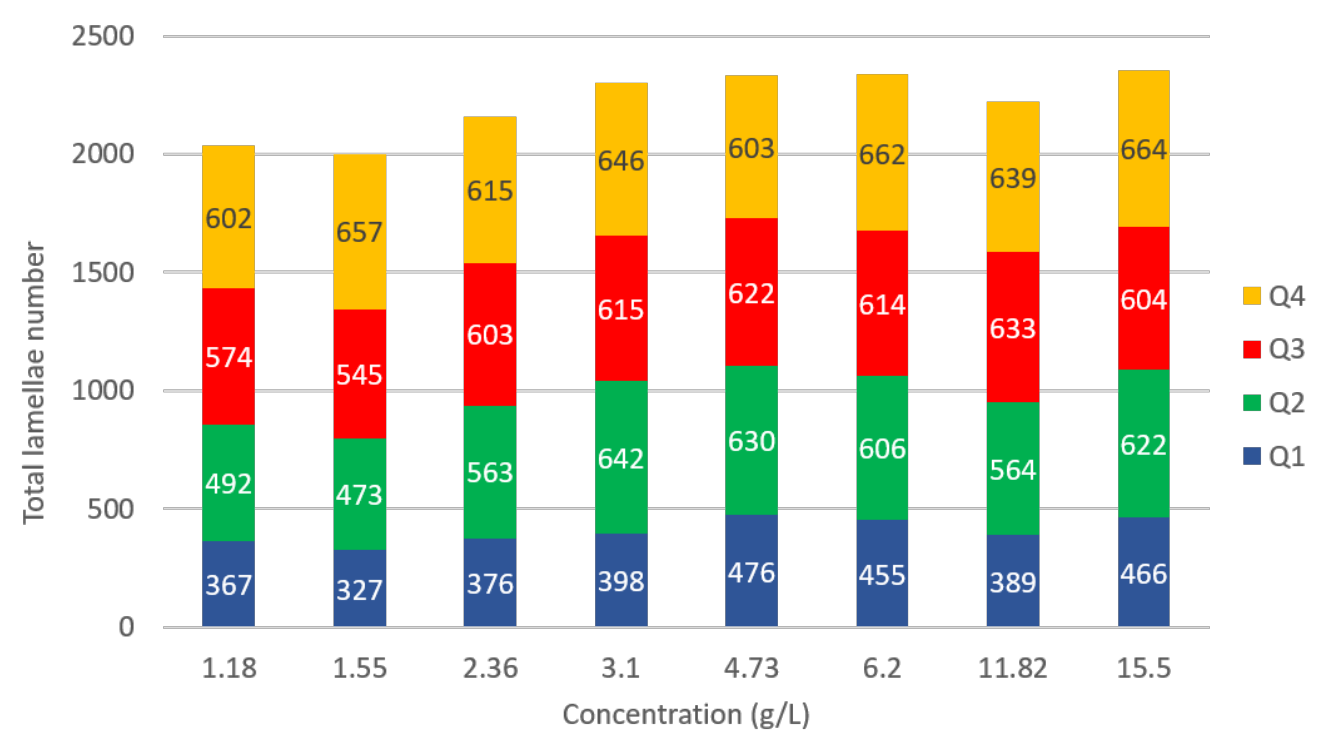

Figure 4.33: Total lamellae number for each concentration as a function of the four regions studied

A higher concentration of lamellae can be observed in the final portion of the micromodel, closer to the exit. In almost all experiments the number 
was maximum at the last quarter Q4, representing around $30 \%$ of the total lamellae number. The total lamellae numbers were between 2002 and 2356 that are compatibles with the values found in the observation area during transient analysis. The micromodel has $200 \mathrm{~mm}^{2}$ and the values at the equilibrium in Figure 4.30 were around 10 and $12 \mathrm{~N} / \mathrm{mm}^{2}$.

The finer texture near the exit can be explained by lamella division. The lamellae created inside the micromodel are multiplied due to the porous media geometry.

\subsection{3}

\section{Reproducibility}

The reproducibility of these experiments was confirmed running the experiment at the same conditions and comparing the measured inlet pressure and lamellae density. The experiments at $\mathrm{c}=1.55 \mathrm{~g} / \mathrm{L}$ and $\mathrm{c}=15.5 \mathrm{~g} / \mathrm{L}$ are presented in Figure 4.34, which shows the inlet pressure, and Figure 4.35, which shows the lamellae density.

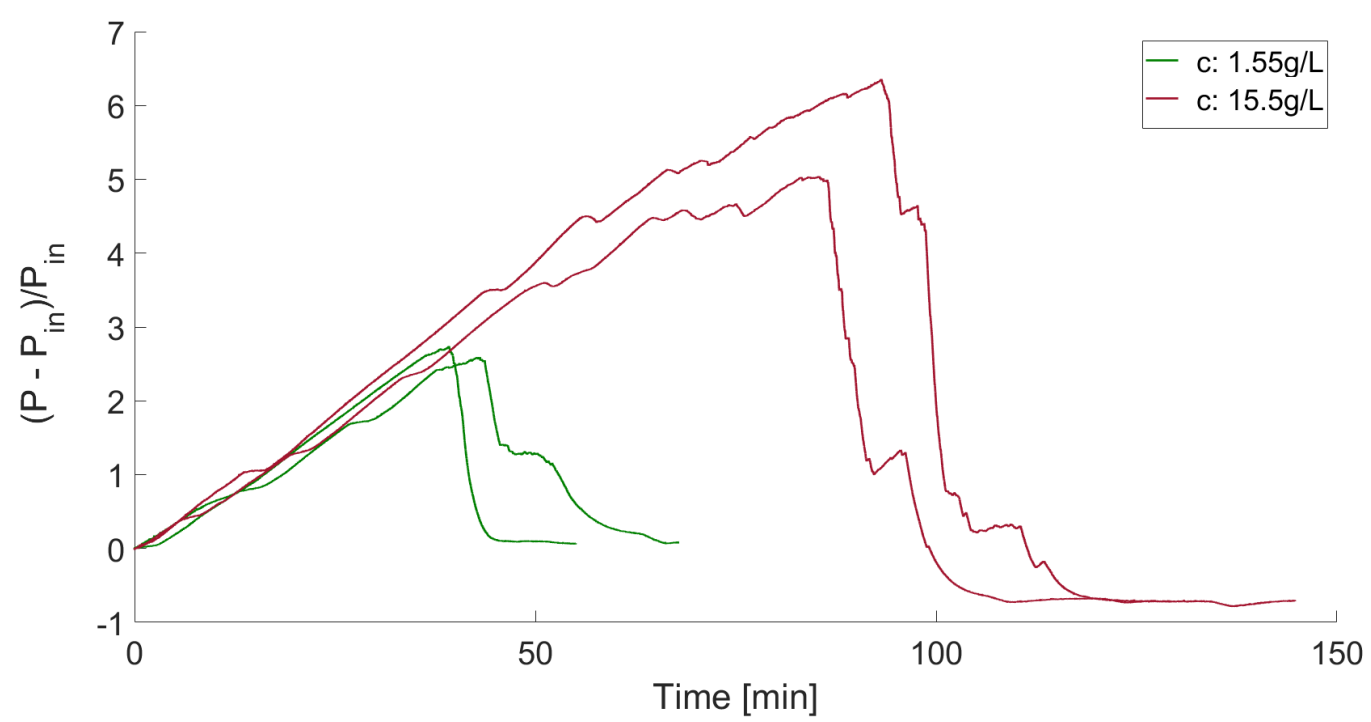

Figure 4.34: Normalized pressure for low surfactant concentration (green curves) and high surfactant concentration (dark red curves) as a function of time 


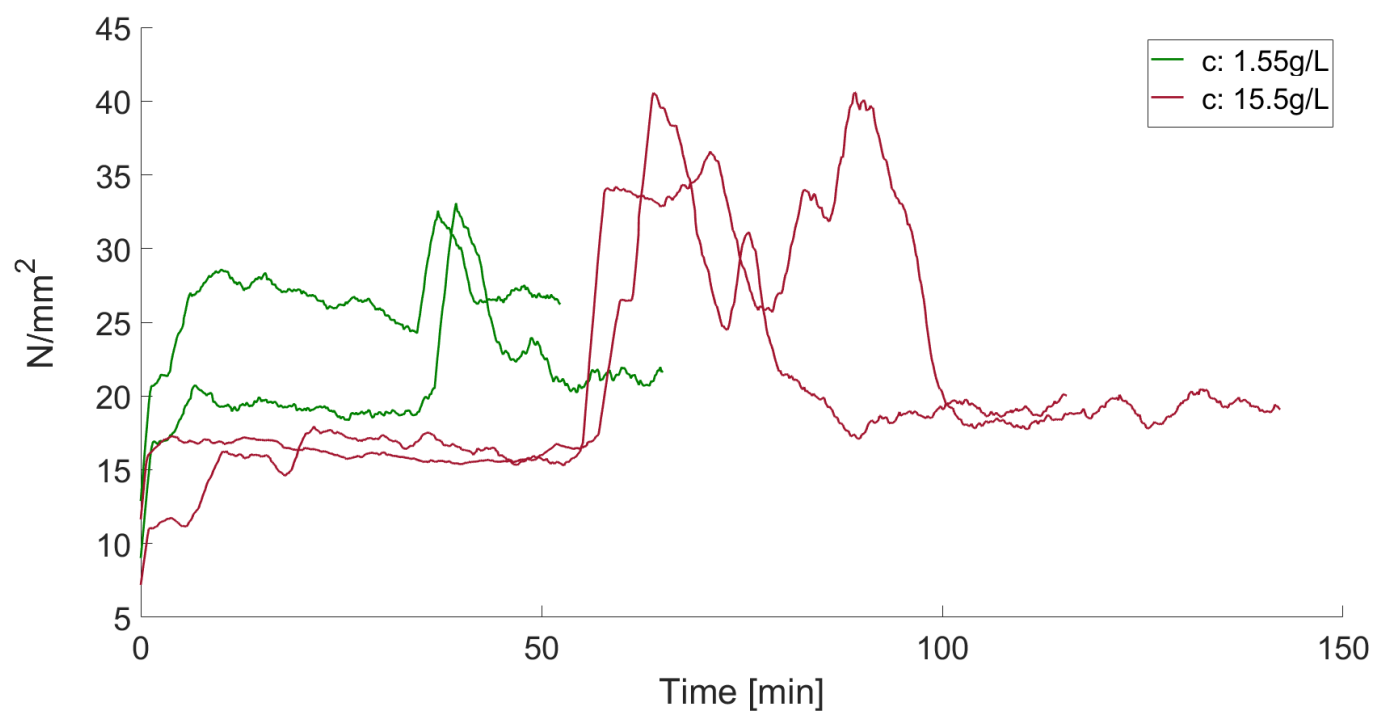

Figure 4.35: Lamellae number per millimeter square for low surfactant concentration (green curves) and high surfactant concentration (dark red curves) as a function of time

The dynamics of the experiments at the same conditions are very similar.

Table 4.1 summarizes the main features of the inlet pressure and lamellae density evolution curves; i.e. maximum and steady state inlet pressures and lamellae density.

Analyzing the numbers in Table 4.1 we can verify that the maximum pressure values are similar (around 3 and $6 \mathrm{psi}$ ) as well as the maximum lamellae number (around 33 and 40 lamellae per millimeter square). The idea is to prove that in the same conditions, the same amount of lamellae would be produced, generating similar resistance for the gas to flow.

\begin{tabular}{c|c|c|c|c|c} 
Test number & $\mathrm{c}[\mathrm{g} / \mathrm{L}]$ & $P_{\max }[\mathrm{psi}]$ & $N_{\max } / \mathrm{mm}^{2}$ & $P_{s s}[\mathrm{psi}]$ & $N_{s s} / \mathrm{mm}^{2}$ \\
\hline 1 & \multirow{2}{*}{1.55} & 3.21 & 32.57 & 0.92 & 25.96 \\
2 & & 3.33 & 33.08 & 1.00 & 21.29 \\
\hline 1 & \multirow{2}{*}{15.5} & 5.48 & 40.57 & 0.26 & 19.67 \\
2 & & 6.02 & 40.58 & 0.24 & 18.93
\end{tabular}

Table 4.1: Experimental values to ratify the test reproducibility

\subsection{4}

\section{Effect of injection flow rate}

According to Chou [58], increasing flow rate accelerates the evolution towards steady state. The same trend was observed in our experiments, as discussed next. 
The experiments reported before were performed at $\mathrm{q}=1 \mathrm{ml} / \mathrm{h}$. The effect of flow rate was studied by repeating the experiments at three concentrations $(\mathrm{c}=1.55 \mathrm{~g} / \mathrm{L}, \mathrm{c}=4.7 \mathrm{~g} / \mathrm{L}$ and $\mathrm{c}=15.5 \mathrm{~g} / \mathrm{L})$ at higher flow rates. Figure 4.36 presents the evolution of the inlet dimensionless pressure as a function of concentration for both flow rates. The pressure at $t=0$ is higher at $q$ $=2 \mathrm{ml} / \mathrm{h}$, as expected.

When comparing experiments with the same concentration in Figure 4.36 , independently of the flow rate, the highest-pressure value achieved is similar.

The main difference is the slope of the curves. The slope is larger at higher gas flow rates $(2 \mathrm{ml} / \mathrm{h})$ and the maximum pressure is reached faster. Using the result at $\mathrm{c}=4.7 \mathrm{~g} / \mathrm{L}$ (yellow curve) as an example, the maximum value for the pressure at both flow rates is around $5 \mathrm{psi}$, but it was reached after approximately $30 \mathrm{~min}$ of gas injection at $2 \mathrm{ml} / \mathrm{h}$ and after approximately $75 \mathrm{~min}$ at $1 \mathrm{ml} / \mathrm{h}$.

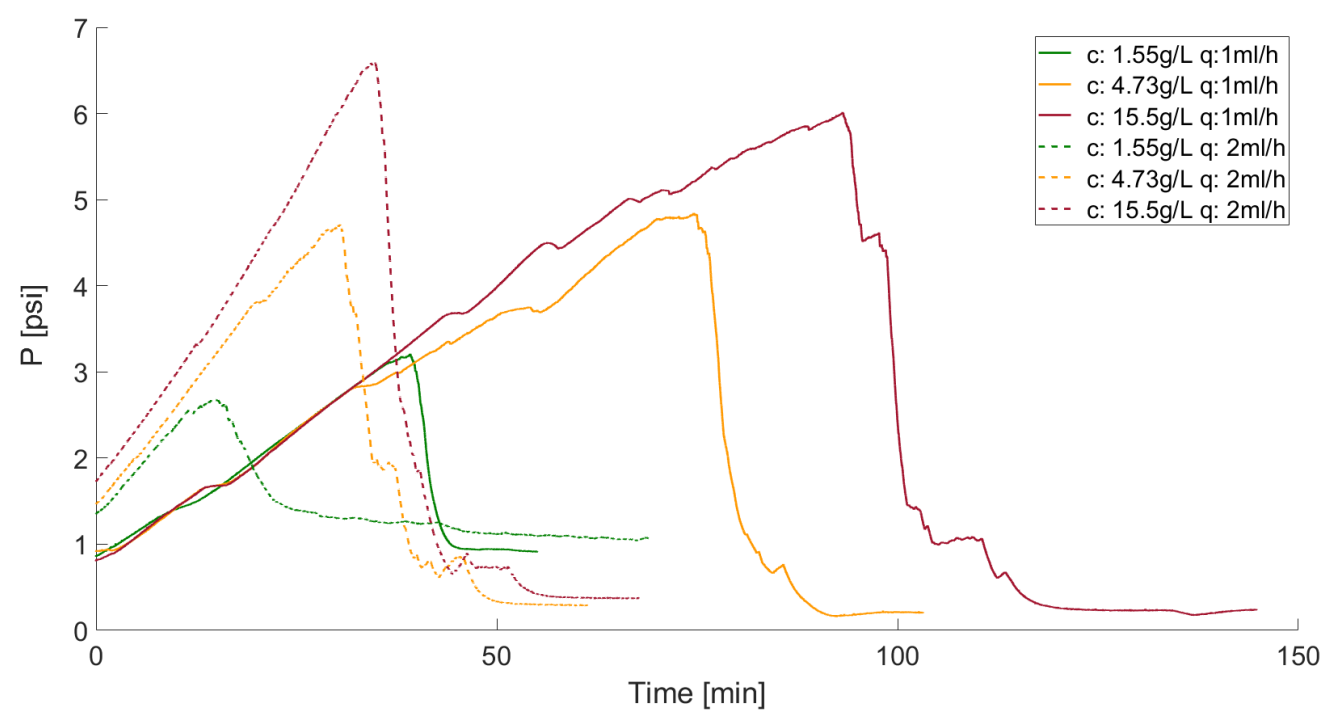

Figure 4.36: Pressure as a function of time during gas injection - different gas flow rates

Figure 4.37 shows the dimensionless pressure behavior. It is possible to observe that the slope of the dimensionless pressure is the same for both flow rates. Nonetheless, the maximum dimensionless pressure value is lower for higher flow rates (because of the higher initial inlet pressure). 


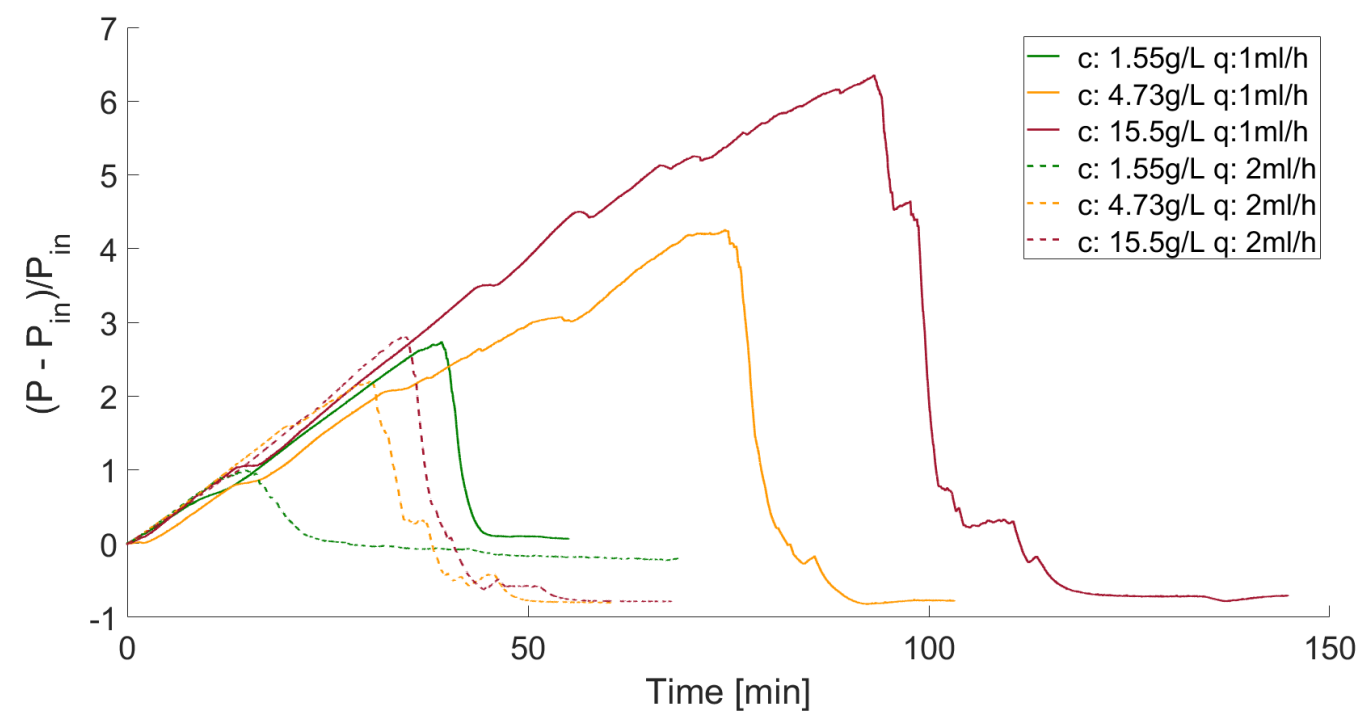

Figure 4.37: Normalized pressure as a function of time during gas injection different gas flow rates

The pressure value at steady state exhibits the same behavior, as shown in detail in Figure 4.38. The values are similar for each concentration, regardless of the flow rate. The pressure at steady state for concentrations near CMC $(0.7 \times \mathrm{CMC})$ is $\bar{P} \approx 0$ and for concentrations of $2 \times \mathrm{CMC}$ and $6.5 \times \mathrm{CMC}$ is $\bar{P} \approx-0.8$.

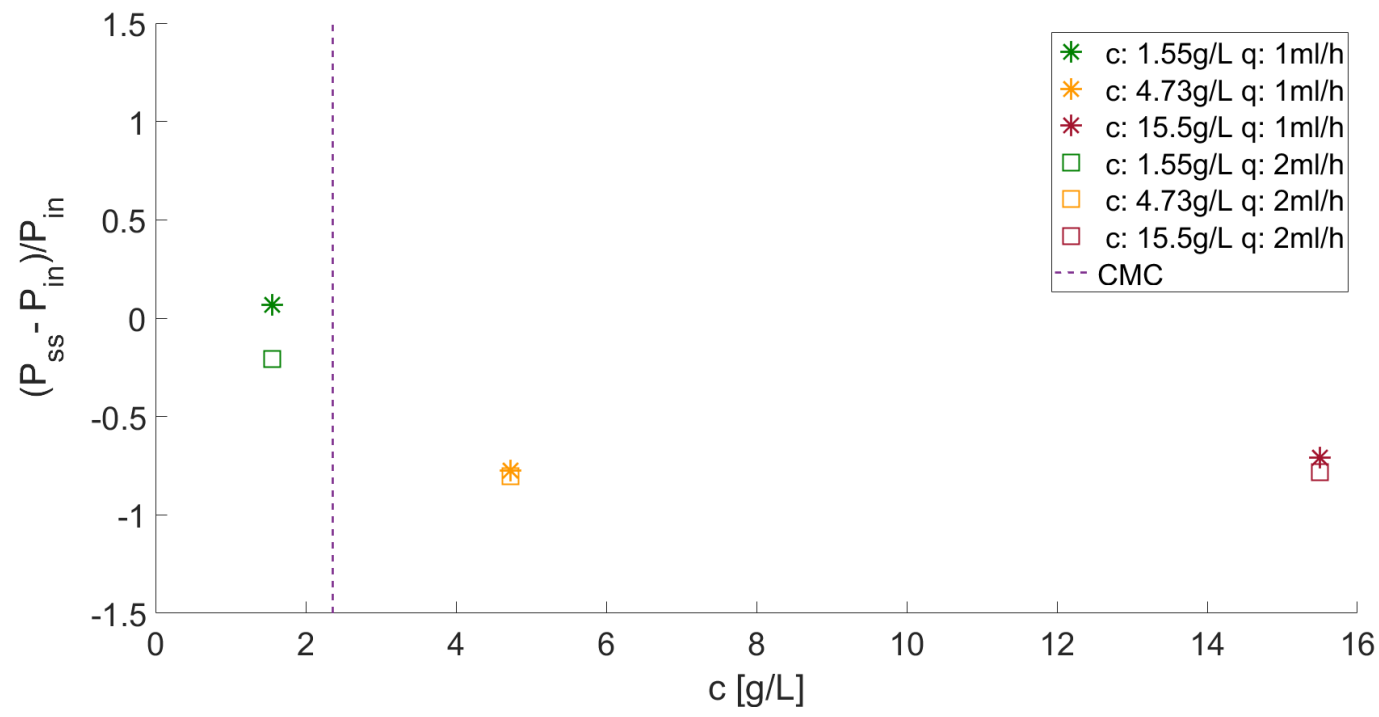

Figure 4.38: Normalized pressure at steady state conditions for different flow rates 


\section{5 \\ Oil displacement by surfactant-alternating-gas (SAG) injec- tion}

To better understand the pore scale dynamics of oil displacement during alternated injection of surfactant solution and gas, three-phase flow experiments were conducted using the glass micromodel.

\section{1}

\section{Experimental setup and procedure}

The experimental setup (Figure 5.1) consisted of a syringe pump (Harvard Apparatus) used for injecting the fluids (aqueous and gas phases) through gas-tight glass syringes (Hamilton). A three-way valve was included to connect the pressure acquisition system. The pressure transmitter was a Wika model S10, that have $0.2 \%$ accuracy and measuring range from 0 to 1 bar. A Nikon SMZ745T stereoscope coupled with a $0.5 \times$ reduction lens made it possible the visualization of the entire device.

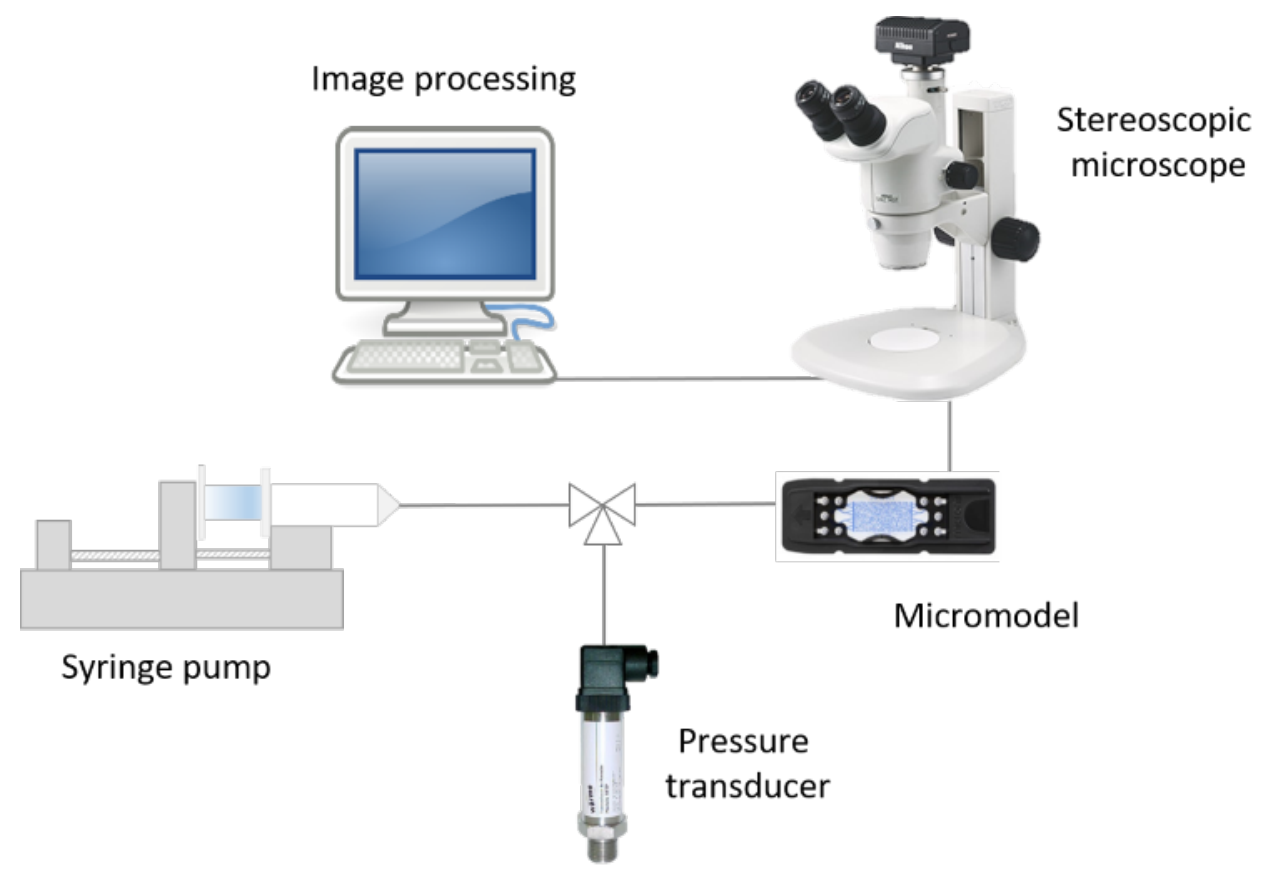

Figure 5.1: Scheme of the experimental setup composed by a syringe pump, a pressure transducer, the micromodel, a stereoscopic microscope and a computer to do the image processing 
The same microfluidic device used in Chapter 4 was saturated with mineral oil. The oil was dyed using a paraffin wax dye (Saramanil) with black color for better visualization. The oil viscosity was $16.5 \mathrm{cP}$ at room temperature.

Water or surfactant solution, dyed in red, was injected in the micromodel using a relatively low flow rate, equal to $0.1 \mathrm{ml} / \mathrm{h}$, as shown in Figure 5.2. Surfactant concentration varied from $0 \mathrm{~g} / \mathrm{L}$ to $15.5 \mathrm{~g} / \mathrm{L}$. After 15 pore-volume (15 PV) of aqueous phase injection, a considerable amount of oil was displaced and a percentage was left in the pore space.

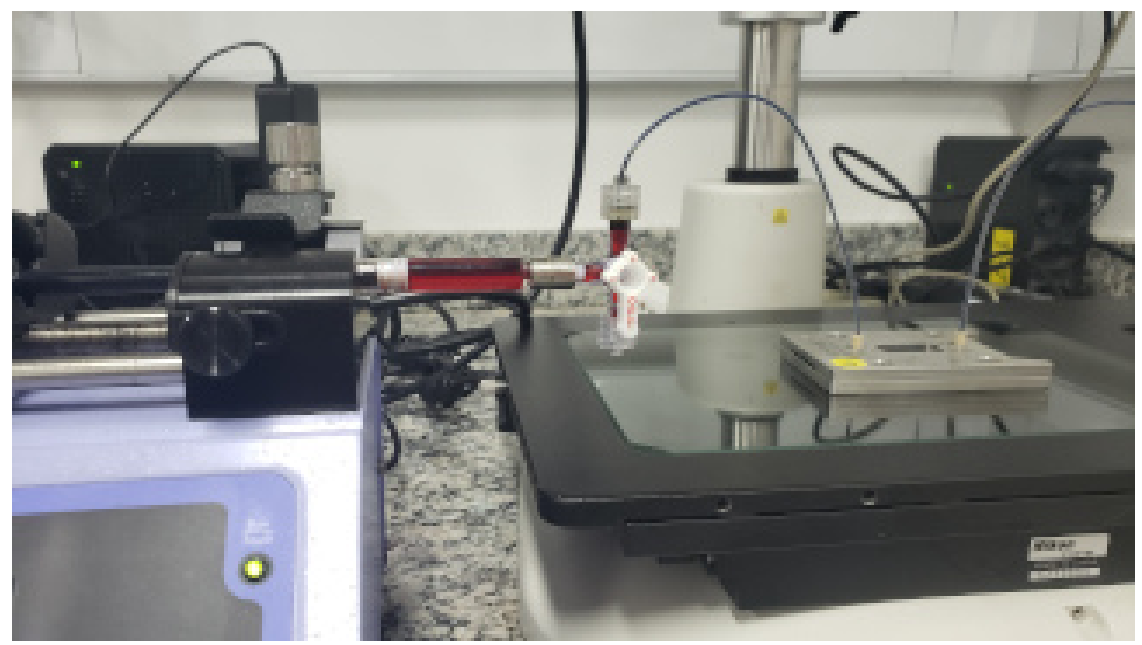

Figure 5.2: Surfactant solution injection in the micromodel saturated with oil

The process continued with gas injection. For the experiments in which the aqueous phase was pure water, the injection of gas mimics a WAG (wateralternating-gas) process. For the cases in which a surfactant solution was used, the process mimics the SAG (surfactant-alternating-gas) process. Air was injected into the porous medium at a constant volumetric flow rate, equal to $2 \mathrm{ml} / \mathrm{h}$, until a steady state was reached.

During gas injection, the inlet pressure was measured and the flow dynamics was visualized using a high-resolution color DS-Fi3 Microscope camera.

\section{2}

\section{Image processing}

ImageJ software was used to evaluate the amount of oil in the pore space at the end of each step of the experimental procedure. The first image analyzed was with the device completely saturated with oil (Figure 5.3(A)). This image was used as the mask of the solid matrix used in the image operation needed to compute oil saturation. 

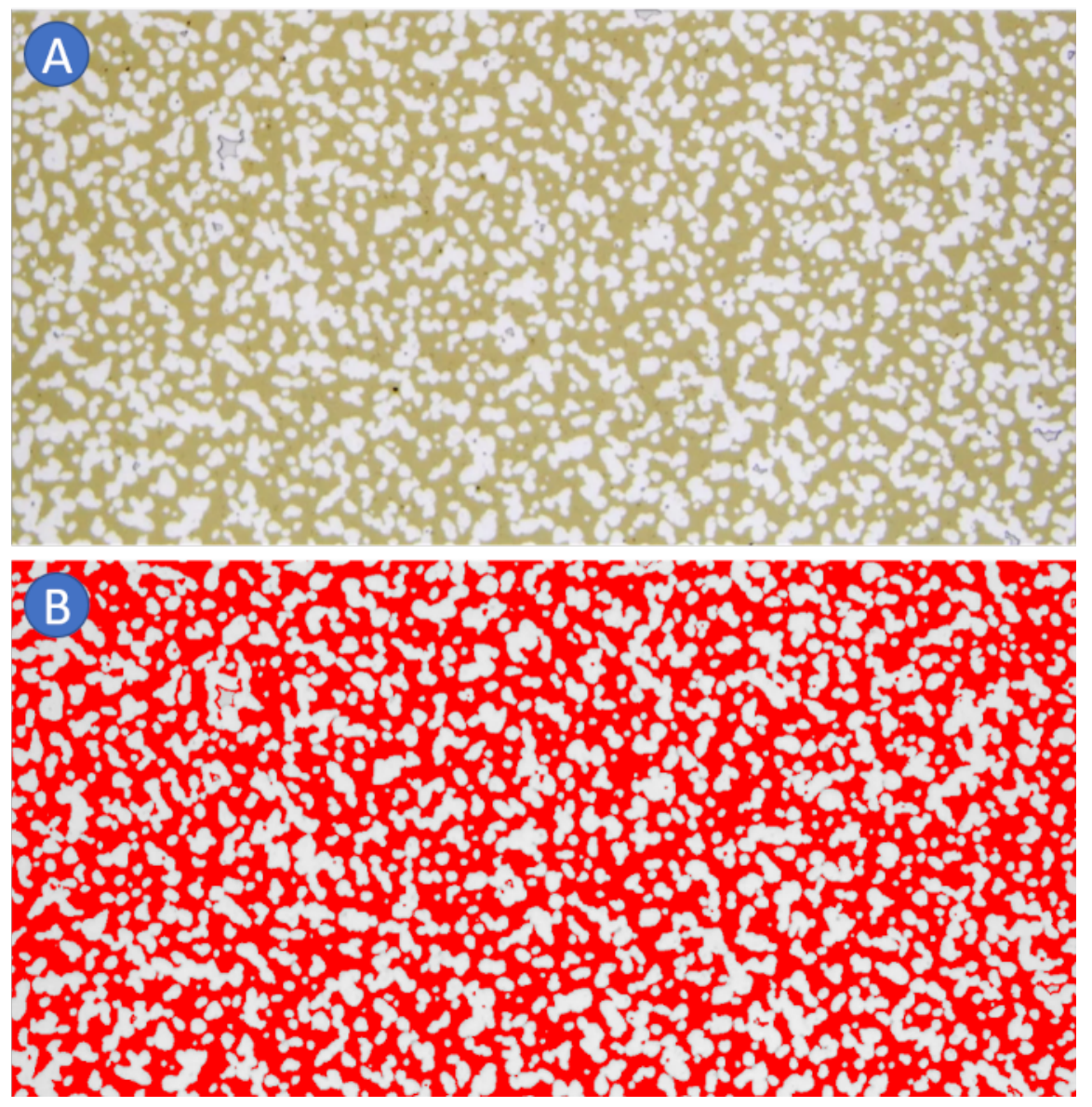

Figure 5.3: Micromodel saturated with oil (A) Original image (B) Mask

The pore space was digitally colored in red by splitting the RGB (red, green, blue) channels to get an image with better contrast, as shown in Figure $5.3(\mathrm{~B})$.

An automatic threshold (Default Threshold) was applied to the image to separate the fluid (pore space) and the glass matrix. Figure 5.4 shows two peeks in the color distribution function corresponding to the shades of gray present in the image. The threshold was used to calculate the percentage of the image occupied by the oil, which corresponded to the pore volume of the device. The calculated porosity was $\varphi=0.55$ that is slightly lower than the data sheet value. 


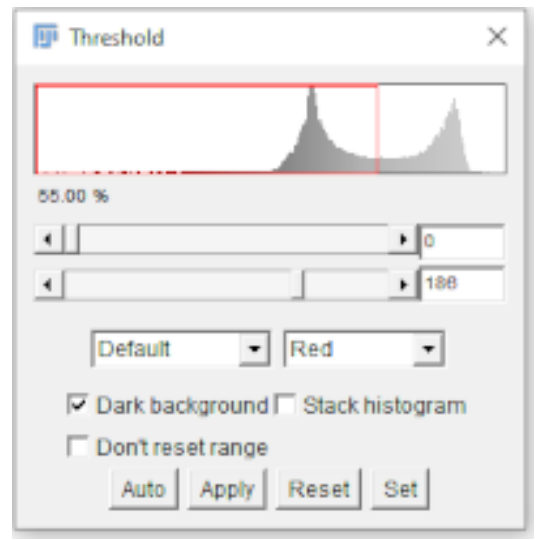

Figure 5.4: Image threshold for the mask

A similar procedure was used to evaluate the oil saturation after the aqueous phase injection. Figure 5.5 shows the pore space occupation after water injection of a typical experiment. The oil corresponds to the brown phase and the aqueous phase corresponds to the red phase. A binary image is obtained after splitting the channels, as shown in Figure 5.6.

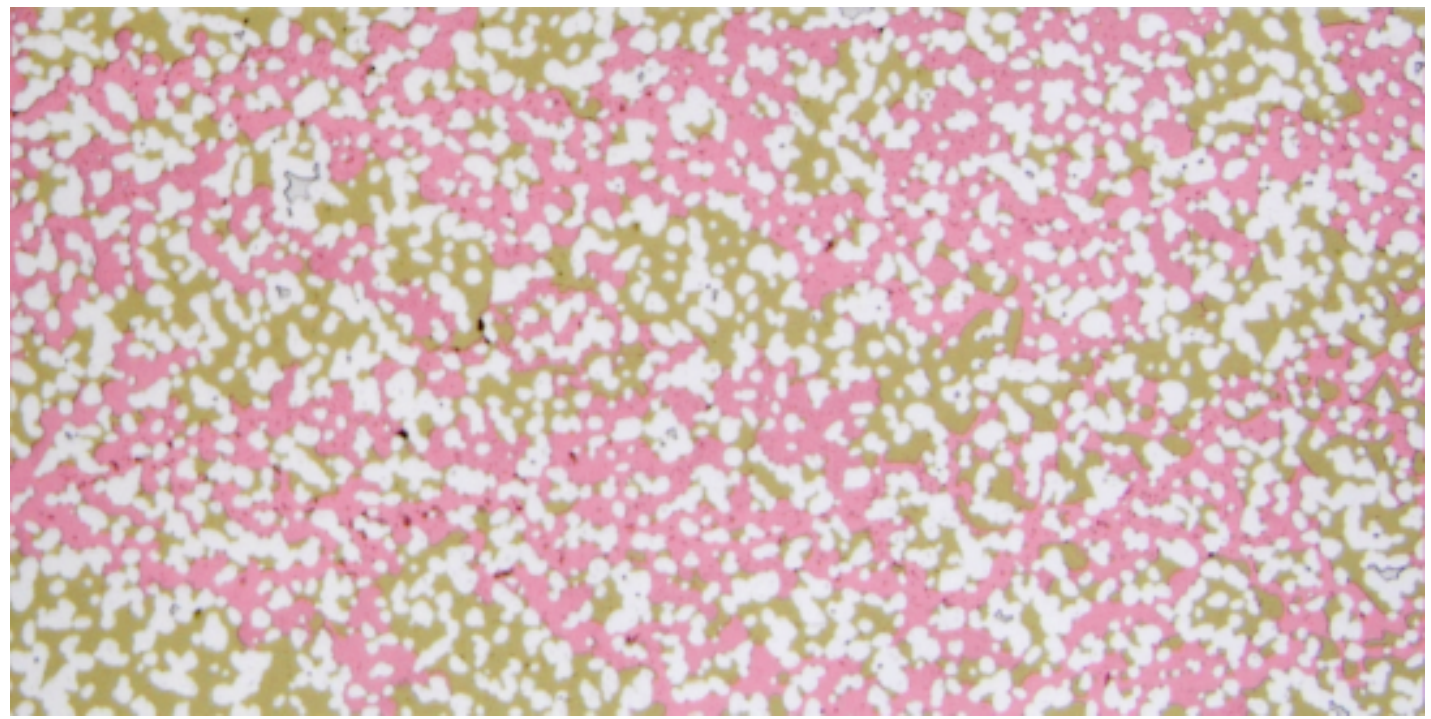

Figure 5.5: Fluid distribution after aqueous solution injection. Oil appears in brown and surfactant solution in red 


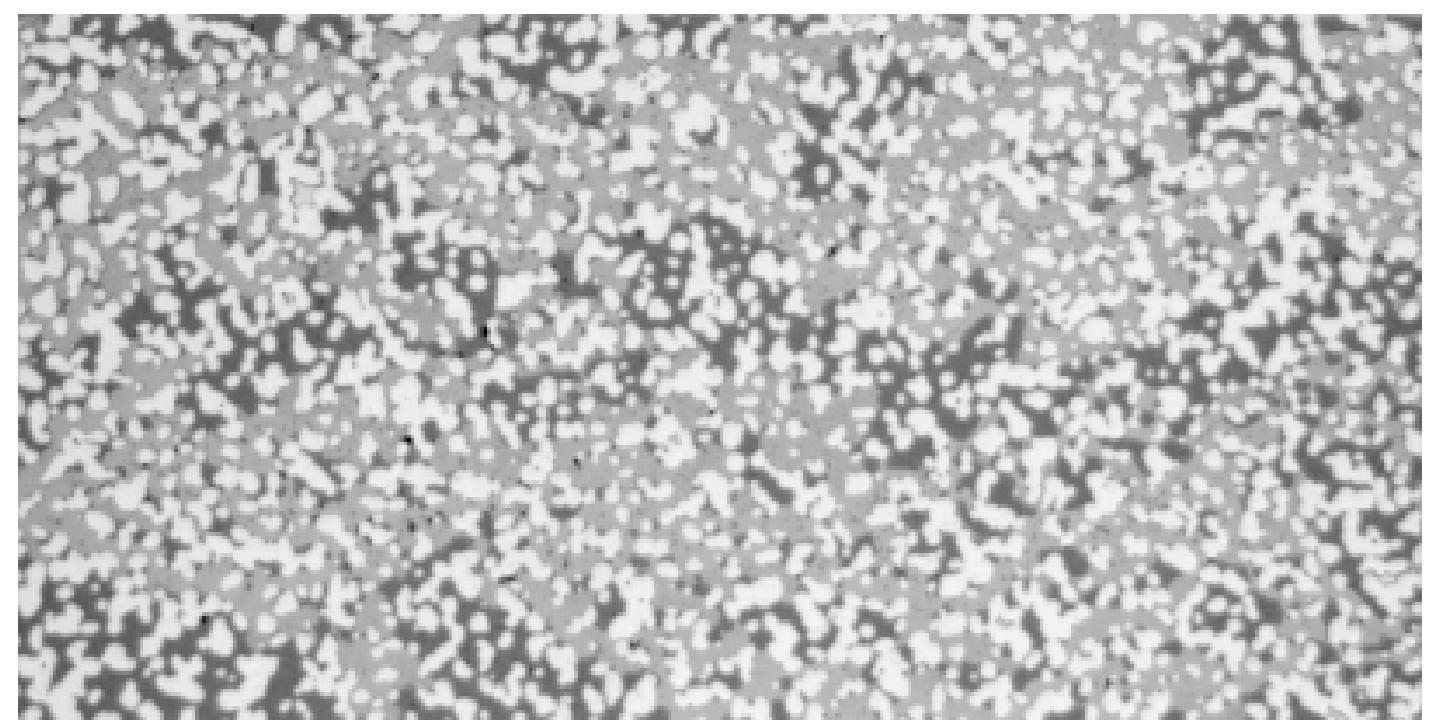

Figure 5.6: Binary image after aqueous solution injection. The darkest regions correspond to the oil phase

The color histogram of the image presented in Figure 5.6 is shown in Figure 5.7. Three peaks can be observed, which correspond to almost white (solid matrix), light gray (water) and dark gray (oil).

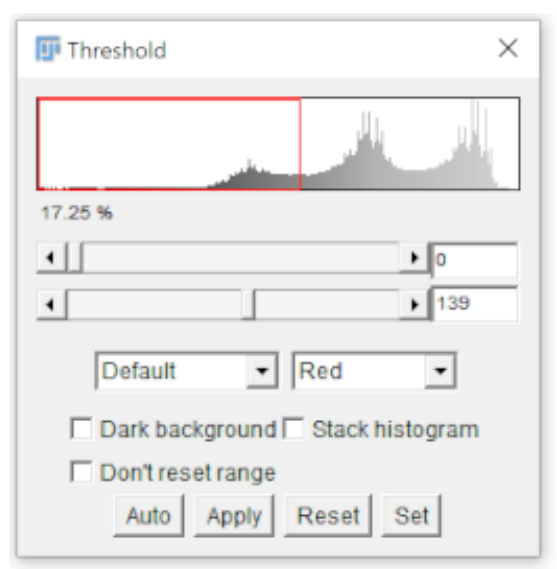

Figure 5.7: Image threshold

Figure 5.8 shows the final image obtained after the threshold selection, in which the oil phase appears in red color. At this point, the remaining oil saturation can be computed. 


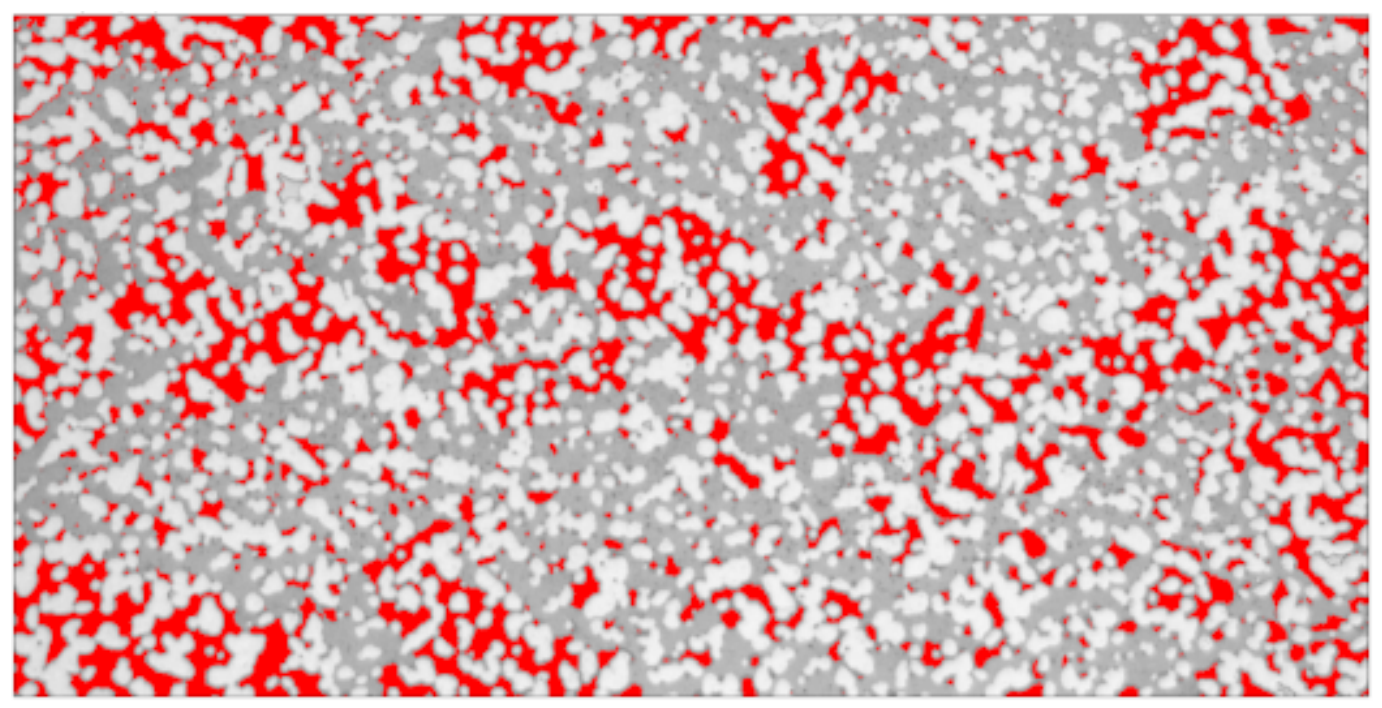

Figure 5.8: Oil phase identified and marked in red after surfactant solution injection

The same procedure was used for the image taken after gas injection. Figure 5.9 shows one example of the resulting image. Again, the remaining oil saturation can be computed.

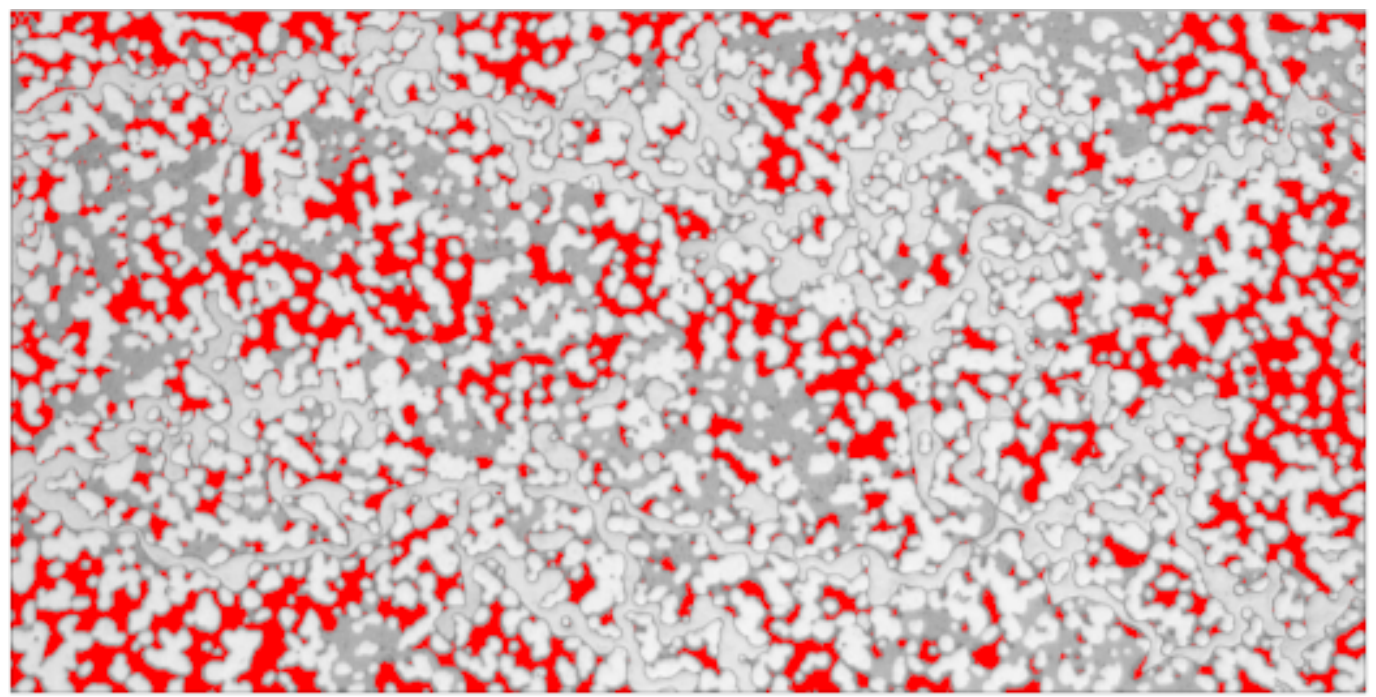

Figure 5.9: Oil phase identified and marked in red after surfactant solution and gas injection

\section{3 \\ Results}

The oil displacement by SAG experiments were designed to investigate the impact of the presence of surfactant and its concentration on the efficiency of oil displacement by foam. They were carried out following the procedures presented in section 5.1. 
Oil displacement by water-alternating-gas (WAG) injection was first analyzed and used as a base case. Figure 5.10 shows the phase distribution in the pore space at the end of pure water injection (Figure 5.10(A)), after 15 PV, and gas (Figure 5.10(B)), after $800 \mathrm{PV}$. The water injection was carried out at a flow rate of $0.1 \mathrm{ml} / \mathrm{h}$, corresponding to a capillary number of $5 \times 10^{-6}$ and viscosity ratio $\mu_{o} / \mu_{w}=17.5$. The water phase is the red phase in the images. Oil is the darker (brown) phase.
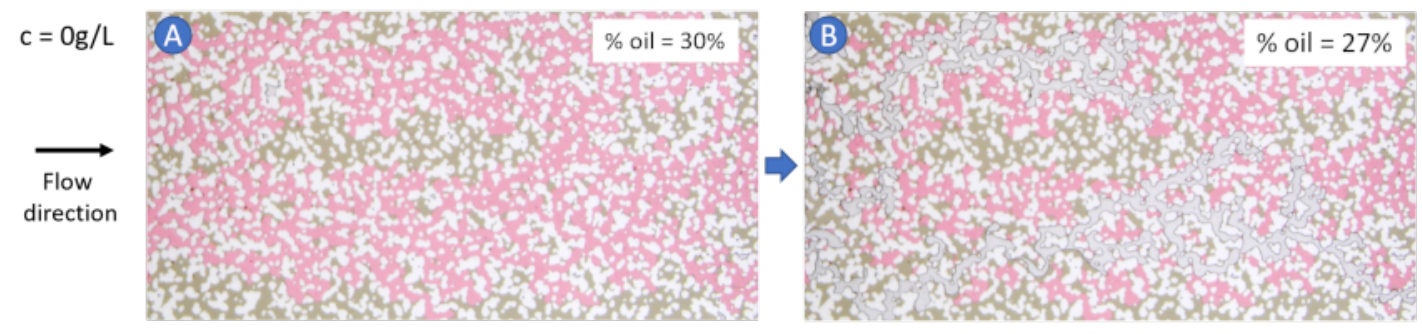

Aqueous phase Oil

Figure 5.10: Fluid saturation after (A) Pure water injection (B) Gas injection

At the end of water injection, oil remains trapped in several regions of the micromodel. The remaining oil saturation was approximately $S_{o} \approx 0.30$. Gas injection was done at $2 \mathrm{ml} / \mathrm{h}$ to guarantee foam formation during the experiment. The path taken by the gas phase is through the water-filled pores and the amount of oil displaced during gas injection is small. The remaining oil saturation after the injection of 800 pore volumes of gas was $S_{o} \approx 0.27$.

The evolution of the gas inlet pressure is presented in Figure 5.11. In the early stages of gas injection, the pressure rises, reaches a maximum value 11 minutes after the beginning of injection, which corresponds to 160 pore volumes, and then stabilizes, achieving a steady state condition. 


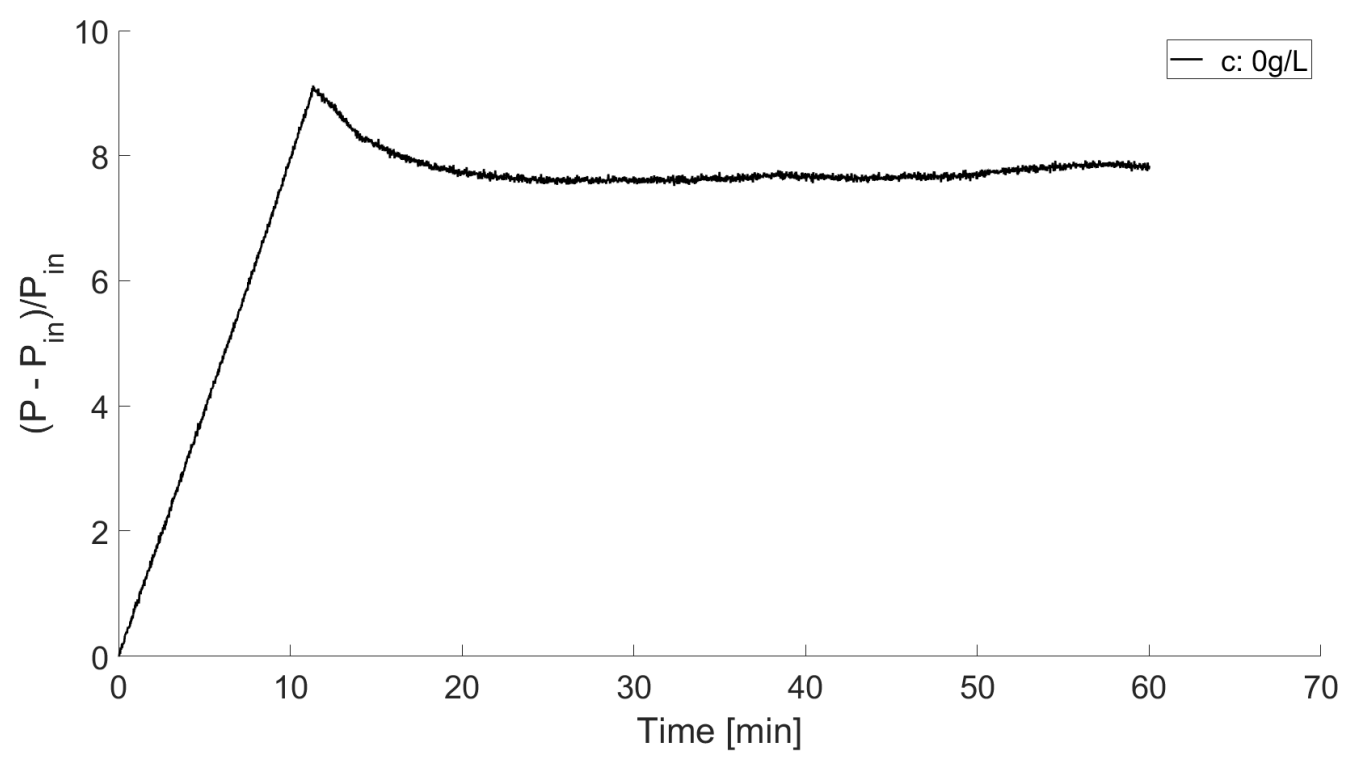

Figure 5.11: Normalized pressure during gas injection

The next experiments show the relevance of adding surfactant to the injected water and the impact of surfactant concentration on foam formation, flow diversion and consequent oil displacement. A concentration of $0.47 \mathrm{~g} / \mathrm{L}$ of SDS was added to the injection water. The surfactant solution was then injected into the porous medium, which was completely saturated with oil. The injection rate used was also $0.1 \mathrm{ml} / \mathrm{h}$. This value of flow rate corresponds to a capillary number $\mathrm{Ca}=3.1 \times 10^{-5}$. The viscosity ratio was $\mu_{o} / \mu_{w}=17.8$. Next, air was injected with a gas-tight syringe into the micromodel at a flow rate of $2 \mathrm{ml} / \mathrm{h}$.

Figure 5.12 shows phase distribution at the end of surfactant solution injection (A), after $15 \mathrm{PV}$, and gas (B), after $800 \mathrm{PV}$. Steady state was achieved after the injection of 200 pore volumes of the gas phase. The remaining oil saturation after the injection of the surfactant solution was approximately the same as after water injection, $S_{o} \approx 0.32$, despite the higher capillary number. Analyzing the images in detail, it is possible to note that the gas flows through pores occupied by the surfactant solution, with very weak flow diversion. The extra oil recovery after the injection of 800 pore volumes of gas was only $\Delta S_{o}$ $\approx 0.04$. The amount of surfactant added to the water in this case is not enough to form strong foam inside the micromodel. 


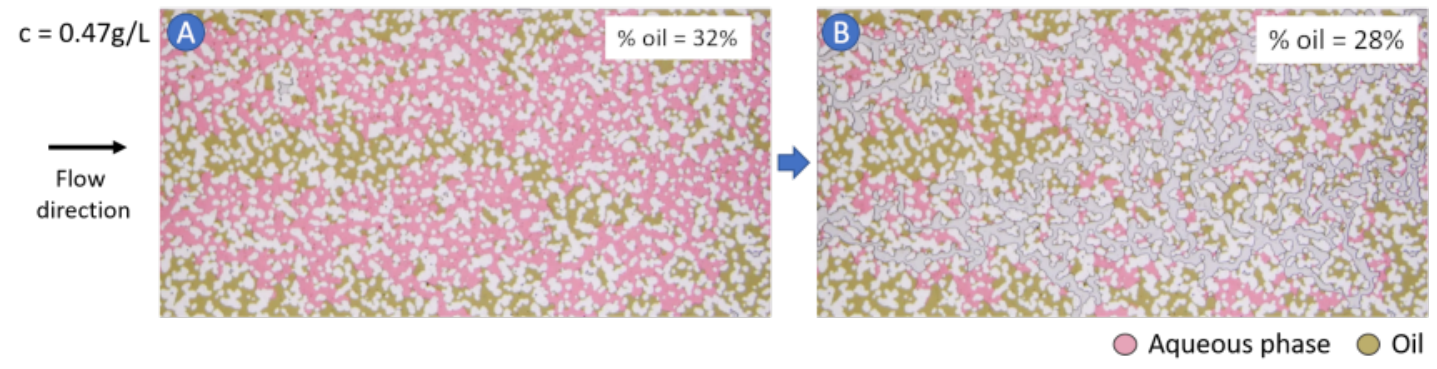

Figure 5.12: Phase distribution after (A) Surfactant solution injection (c= $0.47 \mathrm{~g} / \mathrm{L})(\mathrm{B})$ Gas injection

Pressure behavior during the gas injection is shown in Figure 5.13. The inlet pressure increases until reaching a plateau of $\bar{P} \approx 9$ after approximately 15 min (200 PV). The steady state dimensionless pressure is slightly higher than that obtained in the WAG process, shown in Figure 5.11.

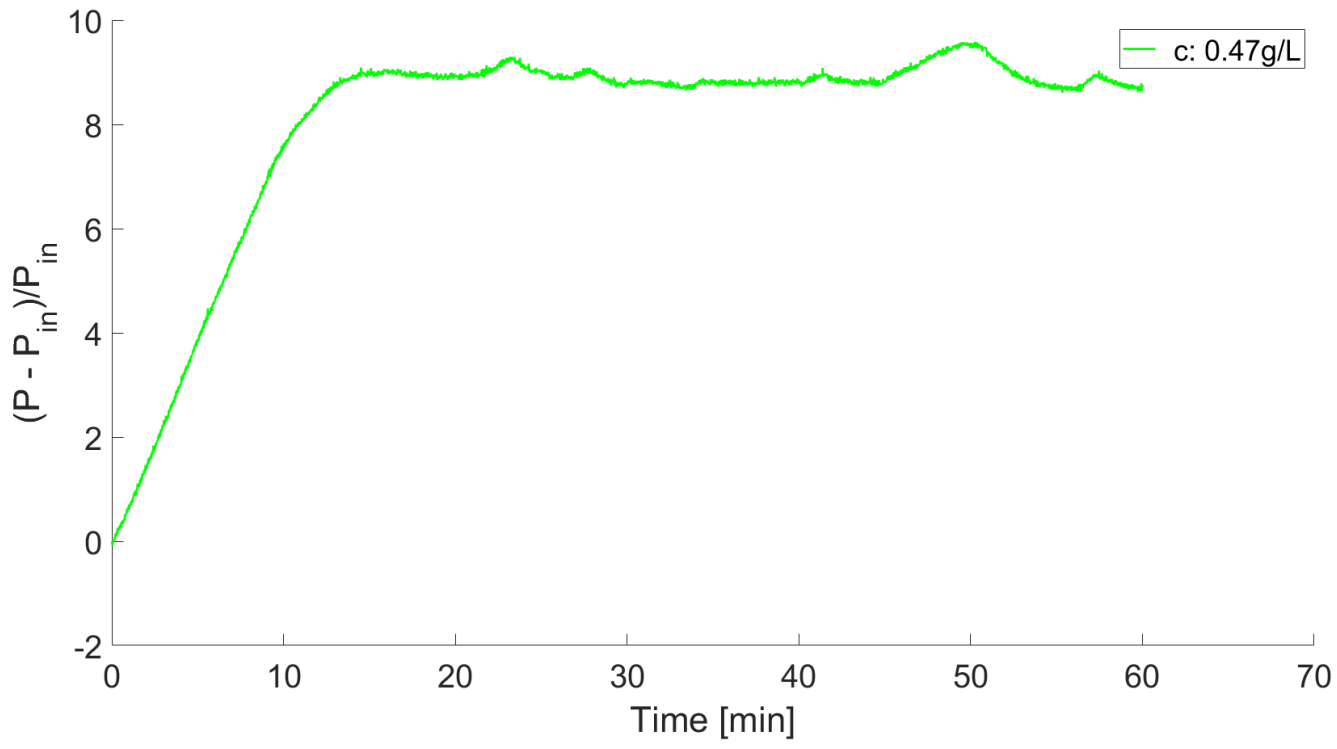

Figure 5.13: Normalized pressure during gas injection (SDS concentration $\mathrm{c}=$ $0.47 \mathrm{~g} / \mathrm{L})$

Experiments were conducted at a concentration of $\mathrm{c}=6.2 \mathrm{~g} / \mathrm{L}, 2.6 \times$ CMC. Figure 5.14 shows phase distribution at the end of surfactant solution injection (A), after $15 \mathrm{PV}$, and gas (B), after $800 \mathrm{PV}$. Steady state was reached after the injection of 600 pore volumes of gas. The injection of the surfactant solution was performed at $\mathrm{Ca}=3.3 \times 10^{-5}$. The remaining oil saturation was similar to that obtained at lower surfactant concentration, $S_{o} \approx$ 0.30. It is possible to notice foam being formed inside the micromodel during gas injection. A large number of lamellae can be seen in Figure 5.14(B). The lamellae created increased flow resistance, thus diverting gas towards oil- 
filled pores and displacing trapped oil. The remaining oil saturation after gas injection was $S_{o} \approx 0.16$, a reduction of $\Delta S_{o} \approx 0.14$.

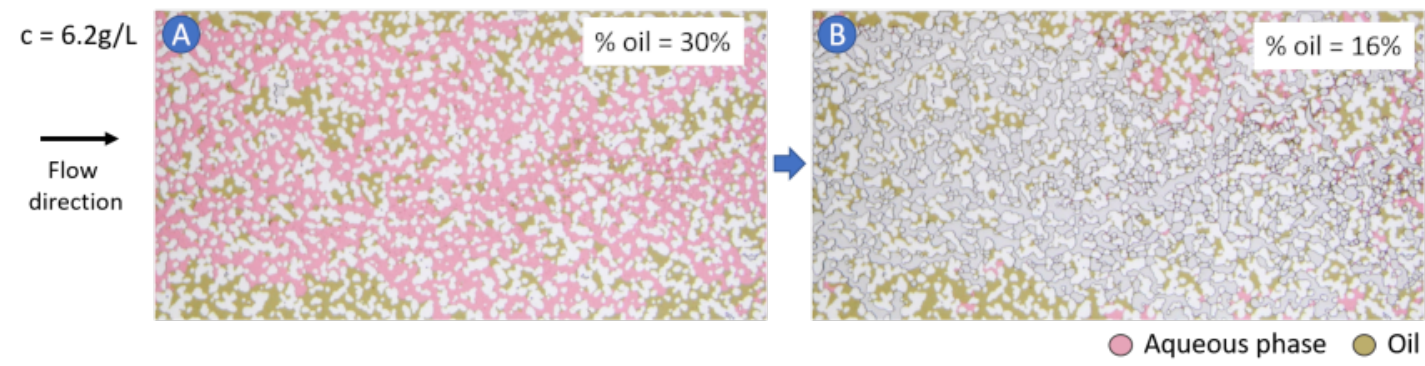

Figure 5.14: Phase distribution after (A) Surfactant solution injection (c = 6.2 $\mathrm{g} / \mathrm{L})(\mathrm{B})$ Gas injection

Figure 5.15 presents the evolution of the dimensionless inlet pressure. The curve shows a peak much higher than previous experiments. The gas inlet pressure reaches a value of $\bar{P} \approx 12$ and then decreases to a plateau at $\bar{P}=6$. The increase in the pressure is explained by the foam formation and the increase in the flow resistance that happens when concentration is higher than the CMC. In this case, the interfacial tension is the same as at the $\mathrm{CMC}$, $\sigma=34.4 \mathrm{mN} / \mathrm{m}$, but the effect caused by foam formation completely changes flow behavior and oil recovery.

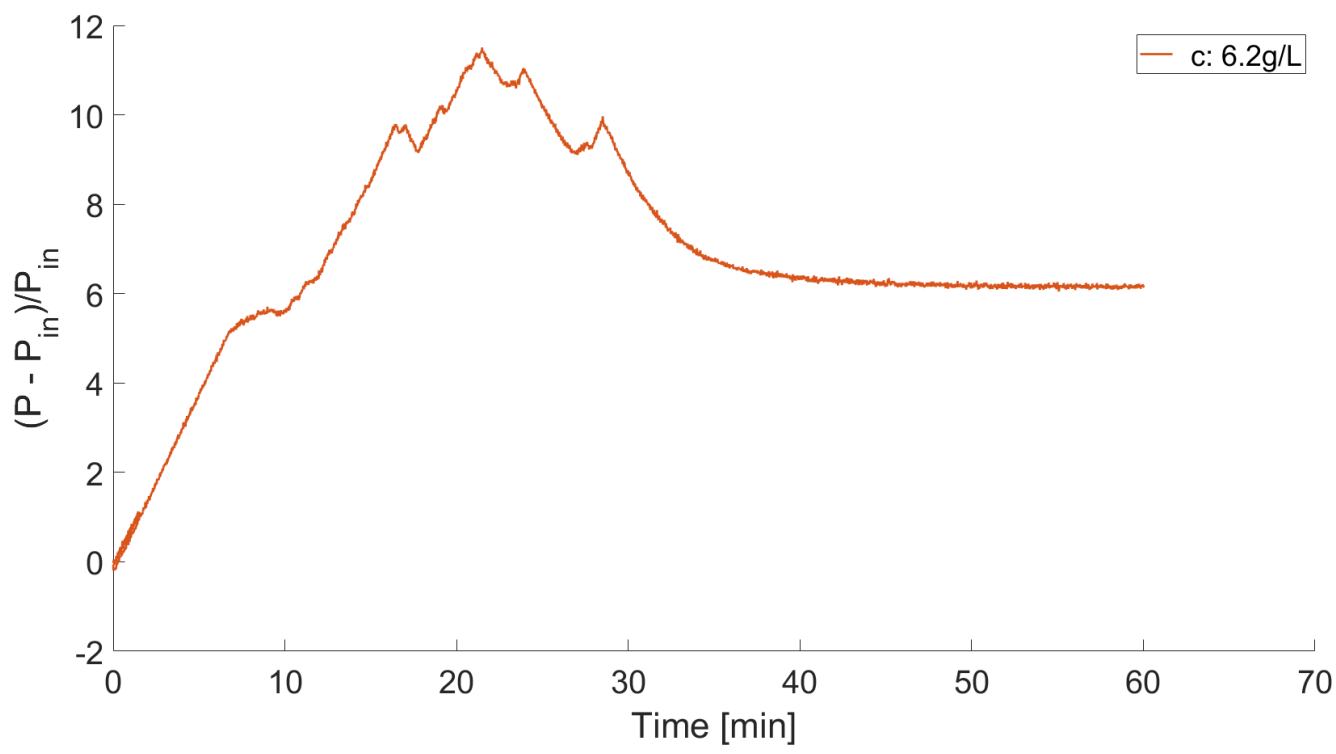

Figure 5.15: Normalized pressure during gas injection (SDS concentration $\mathrm{c}=$ $6.2 \mathrm{~g} / \mathrm{L})$

The effect of foam is even more pronounced if the surfactant concentration is well above the $\mathrm{CMC}, \mathrm{c}=15.5 \mathrm{~g} / \mathrm{L}, 6.5 \times \mathrm{CMC}$. For these experiments, the interfacial tension is also $\sigma=34.4 \mathrm{mN} / \mathrm{m}$. Figure 5.16 shows phase distribution at the end of surfactant solution injection (A), after $15 \mathrm{PV}$, and 
gas injection (B), after $800 \mathrm{PV}$. The injection of the surfactant solution was performed at $\mathrm{Ca}=3.5 \times 10^{-5}$. The remaining oil saturation after surfactant solution injection was $S_{o} \approx 0.18$, lower than the previous experiments, even with the same interfacial tension.

In all the experiments performed, 15 pore volumes of the aqueous phase were injected at a low flow rate $(0.1 \mathrm{ml} / \mathrm{h})$. These parameters were chosen to ensure that there was enough oil in the micromodel prior to gas injection. Thus, these conditions do not guarantee that the steady state regime was reached in all tests after the aqueous phase injection. This could be the explanation for the lower saturation value $\left(S_{o} \approx 0.18\right)$ obtained in the higher surfactant concentration case $(\mathrm{c}=15.5 \mathrm{~g} / \mathrm{L})$. Higher surfactant concentration could also accelerate the reduction of the interfacial tension until reaching steady state value.

Analyzing the Figure 5.16(B), it is possible to see that almost all the surfactant solution available in the micromodel was replaced by foam.

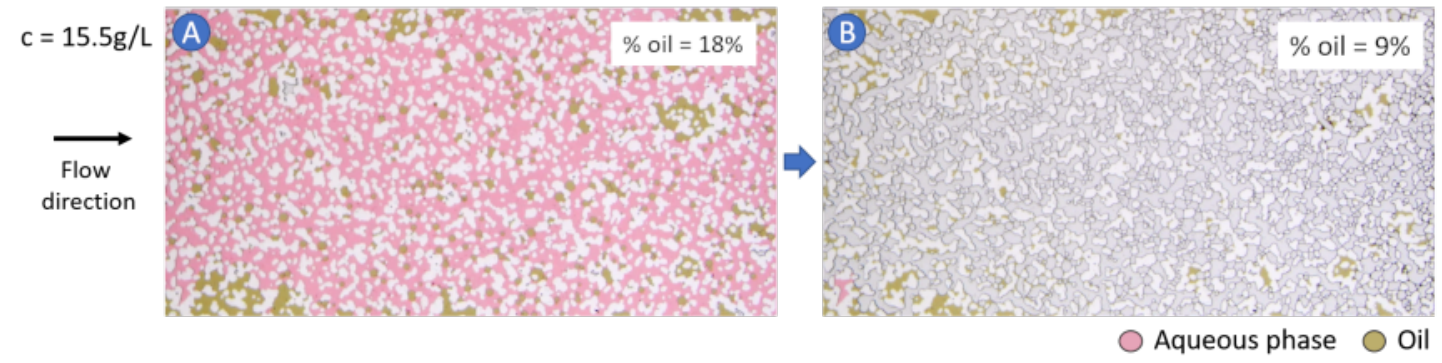

Figure 5.16: Phase distribution after (A) Surfactant solution injection (c= $15.5 \mathrm{~g} / \mathrm{L})(\mathrm{B})$ Gas injection

Figure 5.17 presents the evolution of the dimensionless inlet pressure for $\mathrm{c}=15.5 \mathrm{~g} / \mathrm{L}$. The gas inlet pressure reaches a value of $\bar{P} \approx 19$ after $25 \mathrm{~min}$ (360 PVs) and then falls to a plateau at $\bar{P}=2$ after $40 \mathrm{~min}(600 \mathrm{PVs})$. The high value of the maximum normalized pressure during gas injection explains the higher oil recovery. 


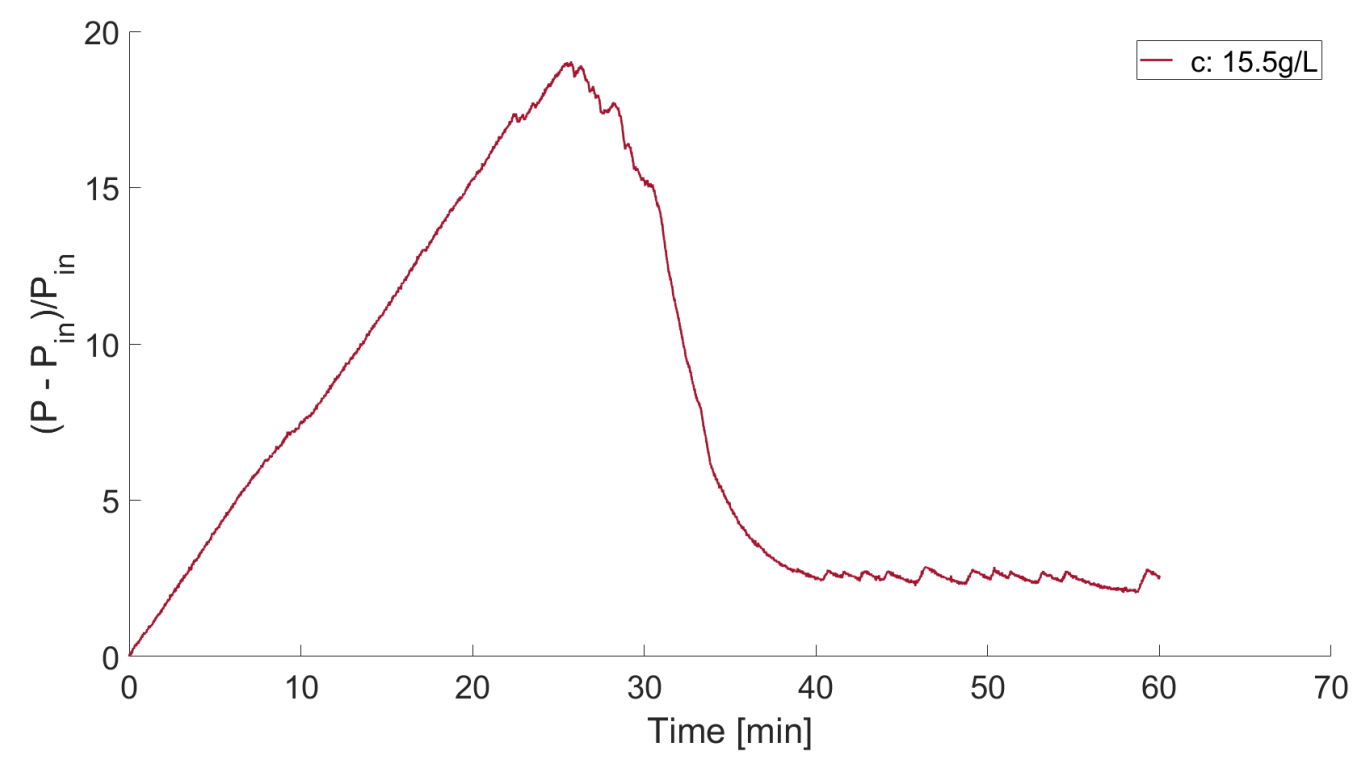

Figure 5.17: Normalized pressure during gas injection (SDS concentration $\mathrm{c}=$ $15.5 \mathrm{~g} / \mathrm{L})$

Figure 5.18 compares the normalized pressure curves for the experiments at different surfactant solution concentrations, previously shown.

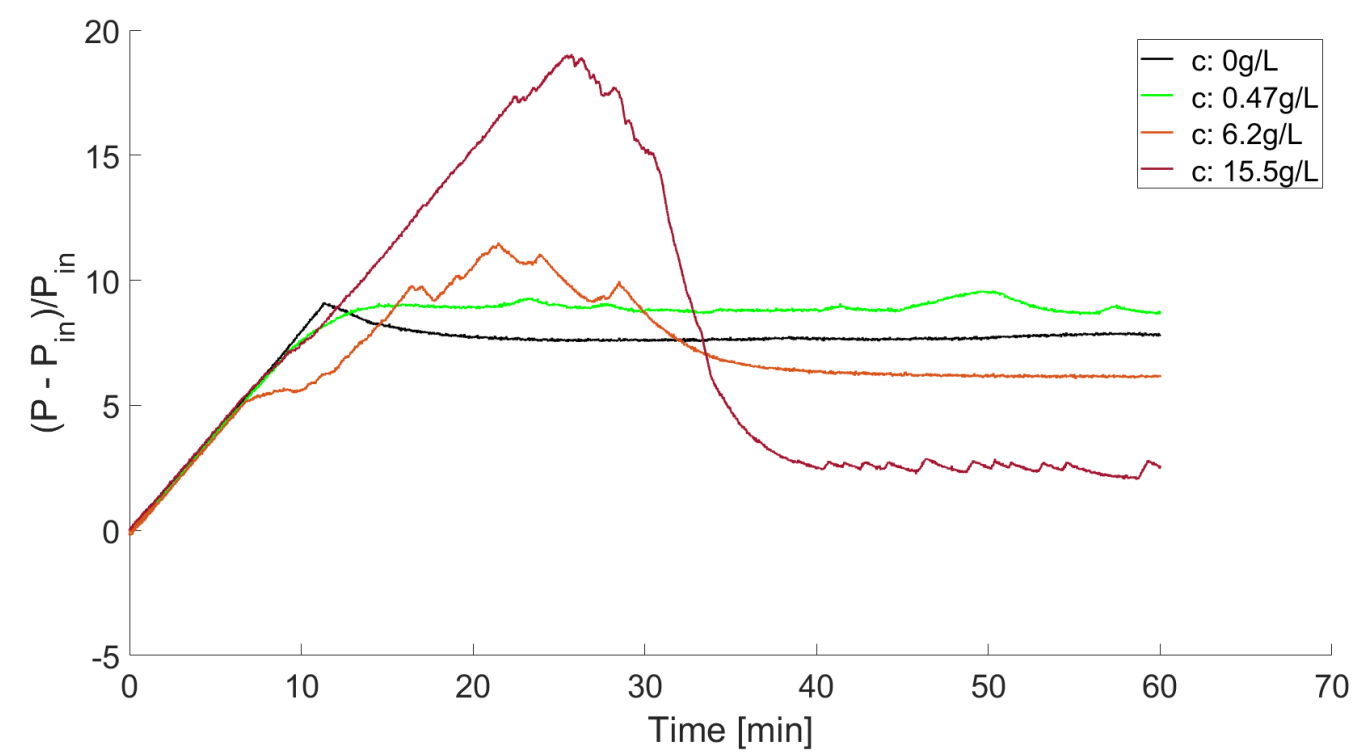

Figure 5.18: Normalized pressure during gas injection for different concentrations after surfactant solution injection

It's possible to note that a high surfactant concentration provides a higher injection pressure associated with a higher number of lamellae being produced, thus, allowing stronger mobility control and more efficient oil displacement.

The remaining oil saturation at the end of each injection step is shown in Table 5.1. The importance of a high surfactant concentration can be noticed. The effectiveness of flow diversion caused by strong foam formed 
at high surfactant concentration is clear. At the highest value of surfactant concentration, the reduction of the remaining oil saturation with respect to that obtained at the end of the aqueous phase injection was close to $50 \%$.

\begin{tabular}{c|c|c|c}
$\mathbf{c}(\mathrm{g} / \mathrm{L})$ & $\begin{array}{c}\text { \% oil remaining after } \\
\mathbf{1 5} \mathbf{P V} \text { aqueous } \\
\text { phase injection }\end{array}$ & $\begin{array}{c}\text { \% oil remaining } \\
\text { after gas injection }\end{array}$ & \% reduction \\
\hline 0 & 29.73 & 26.94 & 9.36 \\
\hline 0.47 & 31.76 & 27.67 & 12.88 \\
\hline 6.2 & 30.2 & 15.51 & 48.65 \\
\hline 15.5 & 18.07 & 8.94 & 50.51
\end{tabular}

Table 5.1: \% of oil available after each step for different concentrations

\subsubsection{1}

\section{Reproducibility}

The reproducibility of the oil displacement experiments was confirmed by comparing the pressure profiles and the remaining oil saturation after gas injection in different experiments performed at same conditions. One example is shown in Figure 5.19 that presents two different experiments with gas flow rate of $2 \mathrm{ml} / \mathrm{h}$ and surfactant concentration was $\mathrm{c}=3.1 \mathrm{~g} / \mathrm{L}$.

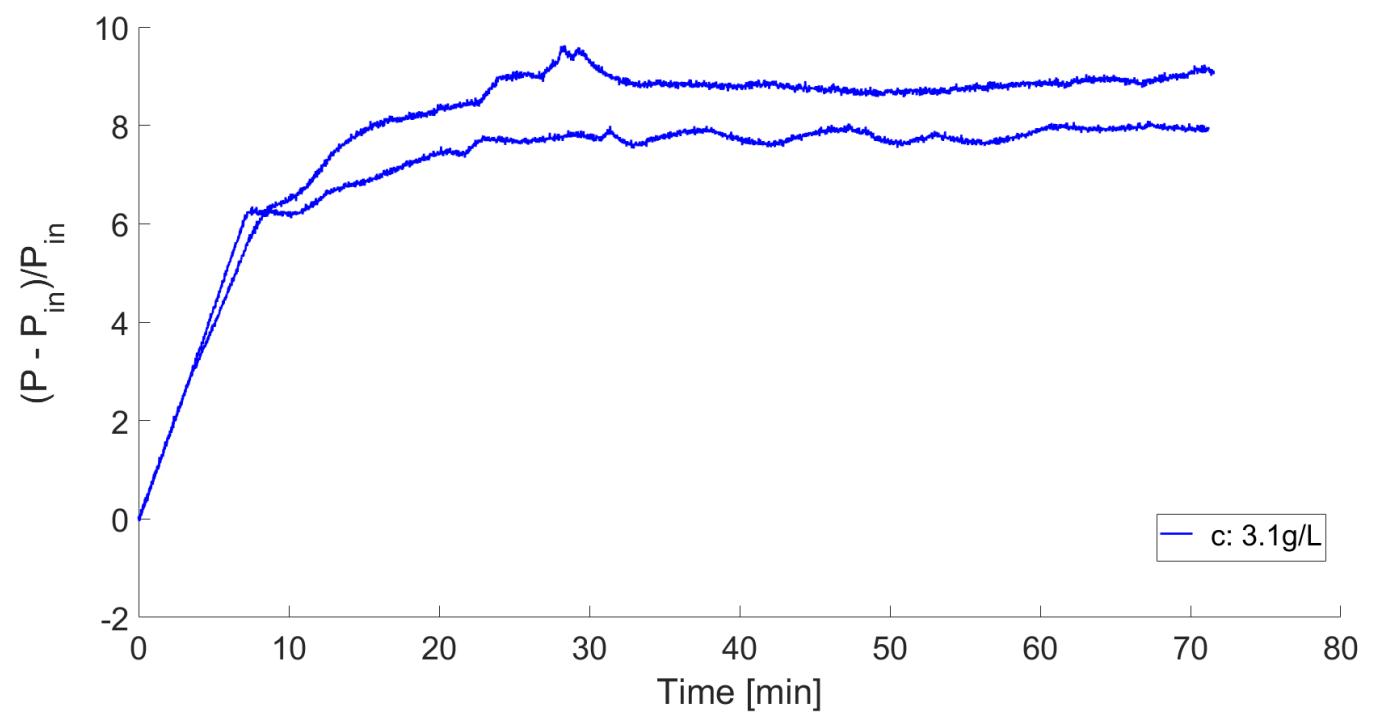

Figure 5.19: Normalized pressure for two different tests at the same concentration as a function of time

The pressure curves show the same behavior as well as the remaining oil saturation in the micromodel device after gas injection. The values in the last column in Table 5.2 are almost the same, approximately $15 \%$. 
Chapter 5. Oil displacement by surfactant-alternating-gas (SAG) injection 92

\begin{tabular}{c|c|c|c} 
c (g/L) & Test number & $\begin{array}{c}\text { \% oil after 15PV } \\
\text { aqueous solution injection }\end{array}$ & $\begin{array}{c}\text { \% oil after } \\
\text { gas injection }\end{array}$ \\
\hline 3.1 & 1 & 21.59 & 15.8 \\
\hline 3.1 & 2 & 17.25 & 15.74
\end{tabular}

Table 5.2: \% of oil available after each step for different experiments at the same concentration 


\section{Conclusions}

In the final chapter of this thesis, the general conclusions drawn from the study of foam formation during surfactant solution drainage by gas and oil displacement during SAG injection using a 2D porous medium are presented. Suggestions for future work are presented in Section 6.3.

\section{1}

\section{Foam formation during drainage of a surfactant solution}

The first study focused on foam formation by injecting gas into a glass micromodel saturated with a surfactant solution. This was a two-phase analysis where an aqueous solution (water or surfactant solution) was injected into the micromodel in order to saturate it, then gas was injected until steady state regime was reached. The data obtained with the experiment was the inlet pressure and a video in a region of interest in the micromodel during the test, and a picture of the entire micromodel at the end of the experiment, after steady state was reached.

From the analysis of the pressure data combined with the dynamic and static lamella count, it was possible to see that the thin films formed (lamellae) create resistance to flow, increasing the magnitude of the viscous forces against capillary forces. The first conclusion is that the pressure behavior does not depend only on the surface tension. The IFT was the same for all experiments above the $\mathrm{CMC}$, but the maximum pressure and the time it occurs increase as a function of the surfactant concentration.

During the experiments, the main mechanisms of foam formation were identified: Leave-behind, Lamella Division, Snap-off and Pinch-off. The repeated occurrence of mechanisms such as Lamella Division and Snap-off was able to multiply the number of lamellae in the micromodel, especially in its final region. A foam destruction mechanism was also identified: Coarsening.

Two regimes based on gas mobility reduction were identified, Weak and Strong foam regimes. In Strong foam regime cases, the critical pressure gradient for foam generation was reached. Stronger foam, has a higher flow resistance that leads to higher inlet pressure. The formation of lamellae generates an 
increase in the apparent viscosity of the foam, leading to a reduction in the gas mobility.

Increasing the surfactant concentration to values well above the CMC dramatically increases the pressure drop. Generating a more effective foam from a continuous gas foam requires mobilizing these lamellae so that they can multiply by lamella division and by repeated snap-off at the unoccupied pore throats.

At higher concentrations (well above the CMC) and high disjoining pressures, more lamellae can be created and a higher resistance is imposed to the gas flow represented by the increase in the pressure gradient.

The areal density of lamellae (number per millimeter square) as a function of time and the total number of lamellae at the end of the experiment were measured. The detailed information on the lamellae formation was related to the macroscopic flow and pressure behavior.

The flow resistance and, consequently, the inlet pressure is directly associated with the number of lamellae formed in the pore space. The rate of lamella formation was very high in the early stages of the displacement process and remains approximately the same for a long time, as inlet pressure rises. A fast increase in the number of lamellae is observed near the maximum pressure. After the maximum value of the inlet pressure is achieved, the number of lamellae drops together with the inlet pressure. After steady state is reached, both the inlet pressure and lamellae density remain almost constant.

A higher concentration of lamellae can be observed in the final portion of the micromodel, closer to the exit. The finer texture near the exit can be explained by lamella division. The lamellae created inside the micromodel are multiplied due to the porous media geometry.

The surfactant concentration changes the population of the lamellae that compose the foam, impeding the free motion of gas through the porous media. Higher concentrations lead to higher lamellae numbers.

The experiments reveal that increasing flow rate accelerates the evolution towards steady state.

\section{2}

\section{Oil displacement by surfactant-alternating-gas (SAG) injection}

The second study presents the results of visualization of pore-scale fluid distribution and displacement mechanisms during oil recovery by SAG injection under conditions of low interfacial tension in a water-wet micromodel. The experiments demonstrate the importance of including surfactant in the 
injected water, as well as the effect of surfactant concentration on foam formation, flow diversion, and oil displacement.

The idea of this study was to simulate an enhanced oil recovery process with the gas injection subsequent to the injection of a surfactant solution in a porous media saturated with oil. This analysis used pressure data as well as visualization of foam formation dynamics during gas injection. The phase saturations were also calculated through image analysis.

For low surfactant concentrations, near the CMC, gas flows through pores occupied by the surfactant solution, with very weak flow diversion. The effects of foam become apparent when the surfactant concentration is increased. For surfactant concentrations well above the CMC, almost all the surfactant solution available in the micromodel was replaced by foam and a considerable amount of oil that has been trapped and left behind after surfactant solution injection is displaced.

The lamellae formed were responsible for increasing flow resistance, diverting gas to oil-filled pores, and displacing trapped oil.

Even though the interfacial tension values above the CMC are the same, the higher amount of surfactant allows a greater number of lamellae to be created.

\section{3}

\section{Future work}

Many aspects of formation and flow of foam through porous media were not explored here.

One important analysis is the evaluation of the lamellae velocity during its formation and correlation between its dynamics with the flow macroscopic behavior.

Another important point is to evaluate the effect of surfactant type. Experiments with cationic, non-ionic and zwitterionic surfactant could be done to compare the foam formation in porous media.

The evolution of the porous space geometry and wettability would also be important aspects to be evaluated as future research. 


\section{Bibliography}

[1] TISTORY. What is waterflooding? (water injection), 2018.

[2] GREEN, D. W.; WILLHITE, G. P. ; OTHERS. Enhanced oil recovery, volumen 6. Henry L. Doherty Memorial Fund of AIME, Society of Petroleum Engineers ..., 1998.

[3] TERRY, R. E.. Enhanced oil recovery. Encyclopedia of physical science and technology, 18:503-518, 2001.

[4] THOMAS, S.. Enhanced oil recovery-an overview. Oil \& Gas Science and Technology-Revue de I'IFP, 63(1):9-19, 2008.

[5] XU, Z.-X.; LI, S.-Y.; LI, B.-F.; CHEN, D.-Q.; LIU, Z.-Y. ; LI, Z.-M.. A review of development methods and eor technologies for carbonate reservoirs. Petroleum Science, 17:990-1013, 2020.

[6] BOUD, D. C.; HOLBROOK, O. C.. Gas drive oil recovery process, Dec. 30 1958. US Patent 2,866,507.

[7] CONN, C. A.; MA, K.; HIRASAKI, G. J. ; BISWAL, S. L.. Visualizing oil displacement with foam in a microfluidic device with permeability contrast. Lab on a Chip, 14(20):3968-3977, 2014.

[8] HIRASAKI, G.; JACKSON, R.; JIN, M.; LAWSON, J.; LONDERGAN, J.; MEINARDUS, H.; MILLER, C.; POPE, G.; SZAFRANSKI, R.; TANZIL, D. ; OTHERS. Field demonstration of the surfactant/foam process for remediation of a heterogeneous aquifer contaminated with dnapl. NAPL Removal: Surfactants, Foams, and Microemulsions, p. 3-163, 2000.

[9] BLAKER, T.; AARRA, M. G.; SKAUGE, A.; RASMUSSEN, L.; CELIUS, H. K.; MARTINSEN, H. A. ; VASSENDEN, F.. Foam for gas mobility control in the snorre field: the fawag project. SPE Reservoir Evaluation \& Engineering, 5(04):317-323, 2002.

[10] PORTOIS, C.; BOEIJE, C. S.; BERTIN, H. J. ; ATTEIA, O.. Foam for environmental remediation: generation and blocking effect. Transport in Porous Media, 124(3):787-801, 2018. 
[11] ZANG, J.; LI, X.; CHEN, Z.; HUANG, L.; LI, Y. ; TELPHI, Y.. An analytical model of foam resistance factor in gas foam flooding. In: SPE NIGERIA ANNUAL INTERNATIONAL CONFERENCE AND EXHIBITION. OnePetro, 2015.

[12] APAYDIN, O. G.; KOVSCEK, A. R.. Surfactant concentration and end effects on foam flow in porous media. Transport in porous media, 43(3):511-536, 2001.

[13] HIRASAKI, G. J.; LAWSON, J.. Mechanisms of foam flow in porous media: apparent viscosity in smooth capillaries. Society of Petroleum Engineers Journal, 25(02):176-190, 1985.

[14] KOVSCEK, A. R.; RADKE, C. J.. Fundamentals of foam transport in porous media. 1993.

[15] TURTA, A. T.; SINGHAL, A. K.. Field foam applications in enhanced oil recovery projects: screening and design aspects. Journal of Canadian Petroleum Technology, 41(10), 2002.

[16] ALLIANCE, T. E.. Gas foams, 2021.

[17] LANGEVIN, D.. Aqueous foams and foam films stabilised by surfactants. gravity-free studies. Comptes Rendus Mécanique, 345(1):47-55, 2017.

[18] CHEN, M.; YORTSOS, Y. ; ROSSEN, W.. Insights on foam generation in porous media from pore-network studies. Colloids and Surfaces A: Physicochemical and Engineering Aspects, 256(2-3):181-189, 2005.

[19] XIAO, S.; ZENG, Y.; VAVRA, E. D.; HE, P.; PUERTO, M.; HIRASAKI, G. J. ; BISWAL, S. L.. Destabilization, propagation, and generation of surfactant-stabilized foam during crude oil displacement in heterogeneous model porous media. Langmuir, 34(3):739-749, 2018.

[20] ROOF, J.; OTHERS. Snap-off of oil droplets in water-wet pores. Society of Petroleum Engineers Journal, 10(01):85-90, 1970.

[21] LIONTAS, R.; MA, K.; HIRASAKI, G. J. ; BISWAL, S. L.. Neighborinduced bubble pinch-off: novel mechanisms of in situ foam generation in microfluidic channels. Soft Matter, 9(46):10971-10984, 2013. 
[22] DHOLKAWALA, Z. F.; SARMA, H. ; KAM, S.. Application of fractional flow theory to foams in porous media. Journal of Petroleum Science and Engineering, 57(1-2):152-165, 2007.

[23] ZHANG, Z.; FREEDMAN, V. L. ; ZHONG, L.. Foam transport in porous media-a review. 2009.

[24] SHENG, J. J.. Enhanced oil recovery field case studies. Gulf Professional Publishing, 2013.

[25] RANSOHOFF, T.; RADKE, C.. Mechanisms of foam generation in glass-bead packs. SPE reservoir engineering, 3(02):573-585, 1988.

[26] ROSSEN, W.; GAUGLITZ, P.. Percolation theory of creation and mobilization of foams in porous media. AIChE Journal, 36(8):11761188, 1990.

[27] TANZIL, D.; HIRASAKI, G. ; MILLER, C.. Conditions for foam generation in homogeneous porous media. paper spe 75176 presented at the spe/doe improved oil recovery symposium, tulsa, oklahoma, 13-17 april 2002, 2002.

[28] GAUGLITZ, P. A.; FRIEDMANN, F.; KAM, S. I. ; ROSSEN, W.. Foam generation in porous media. In: SPE/DOE IMPROVED OIL RECOVERY SYMPOSIUM. OnePetro, 2002.

[29] KAHROBAEI, S.; FARAJZADEH, R.. Insights into effects of surfactant concentration on foam behavior in porous media. In: IOR 2019-20TH EUROPEAN SYMPOSIUM ON IMPROVED OIL RECOVERY, volumen 2019, p. 1-13. European Association of Geoscientists \& Engineers, 2019.

[30] FARAJZADEH, R.; ANDRIANOV, A.; KRASTEV, R.; HIRASAKI, G. ; ROSSEN, W. R.. Foam-oil interaction in porous media: implications for foam assisted enhanced oil recovery. Advances in colloid and interface science, 183:1-13, 2012.

[31] KELAGER, M.; ERLEBEN, K.. A nonlinear vertex-based model for animation of two-dimensional dry foam. In: GRAPP, p. 296-303, 2010.

[32] JONES, S.; GETROUW, N. ; VINCENT-BONNIEU, S.. Foam flow in a model porous medium: I. the effect of foam coarsening. Soft matter, 14(18):3490-3496, 2018. 
[33] LUO, H.; REN, G.; MA, K.; MATEEN, K.; NEILLO, V.; BLONDEAU, C.; BOURDAROT, G. ; MOREL, D.. Importance and inclusion of gas diffusion in co2 emulsion population-balance model. In: IOR 2019-20TH EUROPEAN SYMPOSIUM ON IMPROVED OIL RECOVERY, volumen 2019, p. 1-28. European Association of Geoscientists \& Engineers, 2019.

[34] DERJAGUIN, B.; LANDAU, L.. Theory of the stability of strongly charged lyophobic sols and of the adhesion of strongly charged particles in solutions of electrolytes. J. Exptl. Theoret. Phys, 14:633, 1941.

[35] VERWEY, E. J. W.. Theory of the stability of lyophobic colloids. The Journal of Physical Chemistry, 51(3):631-636, 1947.

[36] DERJAGUIN, B.; CHURAEV, N.. Disjoining pressure of thin layers of binary solutions. Journal of Colloid and Interface Science, 62(3):369-380, 1977.

[37] ARONSON, A.; BERGERON, V.; FAGAN, M. E. ; RADKE, C.. The influence of disjoining pressure on foam stability and flow in porous media. Colloids and Surfaces A: Physicochemical and Engineering Aspects, 83(2):109-120, 1994.

[38] VELIKOV, K.; VELEV, O.; MARINOVA, K. ; CONSTANTINIDES, G.. Effect of the surfactant concentration on the kinetic stability of thin foam and emulsion films. Journal of the Chemical Society, Faraday Transactions, 93(11):2069-2075, 1997.

[39] HÉDREUL, C.; FRENS, G.. Foam stability. Colloids and Surfaces A: Physicochemical and Engineering Aspects, 186(1-2):73-82, 2001.

[40] BeRgeron, V.; WALTERMO, Å. ; CLAESSON, P. M.. Disjoining pressure measurements for foam films stabilized by a nonionic sugar-based surfactant. Langmuir, 12(5):1336-1342, 1996.

[41] ADEBANJO, F. O.; OLUSEGUN, O.. Evaluating the application of foam injection as an enhanced oil recovery in unconsolidated sand. Journal of Petroleum and Gas Engineering, 6(2):22-37, 2015.

[42] BERNARD, G. G.; HOLM, L. ; OTHERS. Effect of foam on permeability of porous media to gas. Society of Petroleum Engineers Journal, 4(03):267-274, 1964. 
[43] FALLS, A.; MUSTERS, J.; RATULOWSKI, J. ; OTHERS. The apparent viscosity of foams in homogeneous bead packs. SPE Reservoir Engineering, 4(02):155-164, 1989.

[44] SALEHI, M. M.; SAFARZADEH, M. A.; SAHRAEI, E. ; NEJAD, S. A. T.. Comparison of oil removal in surfactant alternating gas with water alternating gas, water flooding and gas flooding in secondary oil recovery process. Journal of Petroleum Science and Engineering, 120:86-93, 2014.

[45] KAMAL, M. S.; ADEBAYO, A. R.; FOGANG, L. T. ; BARRI, A. A.. Improving gas sequestration by surfactant-alternating-gas injection: A comparative evaluation of the surfactant type and concentration. Journal of Surfactants and Detergents, 21(5):667-675, 2018.

[46] WANG, L.; HE, Y.; WANG, Q.; LIU, M. ; JIN, X.. Multiphase flow characteristics and eor mechanism of immiscible co2 wateralternating-gas injection after continuous co2 injection: A microscale visual investigation. Fuel, 282:118689, 2020.

[47] LI, Q.; PRIGIOBBE, V.. Studying the generation of foam in the presence of nanoparticles using a microfluidic system. Chemical Engineering Science, 215:115427, 2020.

[48] LI, K.; WOLF, K.-H. A. ; ROSSEN, W. R.. Effects of gas trapping on foam mobility in a model fracture. Transport in Porous Media, 138(1):185-200, 2021.

[49] LIN, S.. A computer simulation and molecular-thermodynamic framework to model the micellization of ionic branched surfactants in aqueous solution. PhD thesis, Massachusetts Institute of Technology, 2008.

[50] PATIST, A.; OH, S.; LEUNG, R. ; SHAH, D.. Kinetics of micellization: its significance to technological processes. Colloids and Surfaces A: Physicochemical and Engineering Aspects, 176(1):3-16, 2001.

[51] EL-HEFIAN, E. A.; YAHAYA, A. H.. Investigation on some properties of sds solutions. Aust. J. Basic Appl. Sci, 5:1221-1227, 2011.

[52] MILES, G. D.; SHEDLOVSKY, L.. Minima in surface tensionconcentration curves of solutions of sodium alcohol sulfates. The journal of physical chemistry, 48(1):57-62, 1944. 
[53] CHIU, Y.; WANG, S.. The micellar dissociation concentration of impure sodium dodecyl sulfate systems in water. Colloids and surfaces, 48:297-309, 1990.

[54] GUNDA, N. S. K.; BERA, B.; KARADIMITRIOU, N. K.; MITRA, S. K. ; HASSANIZADEH, S. M.. Reservoir-on-a-chip (roc): A new paradigm in reservoir engineering. Lab on a Chip, 11(22):3785-3792, 2011.

[55] BUCHgRABER, M.; CLEMENS, T.; CASTANIER, L. M. ; KOVSCEK, A.. A microvisual study of the displacement of viscous oil by polymer solutions. SPE Reservoir Evaluation \& Engineering, 14(03):269-280, 2011.

[56] YUN, W.; CHANG, S.; COGSWELL, D. A.; EICHMANN, S. L.; GIZZATOV, A.; THOMAS, G.; AL-HAZZA, N.; ABDEL-FATTAH, A. ; WANG, W.. Toward reservoir-on-a-chip: rapid performance evaluation of enhanced oil recovery surfactants for carbonate reservoirs using a calcite-coated micromodel. Scientific reports, 10(1):1-12, 2020.

[57] ROSSEN, W. R.; OCAMPO, A.; RESTREPO, A.; CIFUENTES, H. D. ; MARIN, J.. Long-time diversion in surfactant-alternating-gas foam enhanced oil recovery from a field test. SPE Reservoir Evaluation \& Engineering, 20(01):001-007, 2017.

[58] CHOU, S.; OTHERS. Conditions for generating foam in porous media. In: SPE ANNUAL TECHNICAL CONFERENCE AND EXHIBITION. Society of Petroleum Engineers, 1991. 